\title{
HYBRID SILICON MODE-LOCKED LASER WITH IMPROVED RF POWER BY IMPEDANCE MATCHING
}

\author{
A Thesis \\ presented to \\ the Faculty of California Polytechnic State University, \\ San Luis Obispo
}

\author{
In Partial Fulfillment \\ of the Requirements for the Degree \\ Master of Science in Electrical Engineering
}

by

Bassem Tossoun

September 2014 
(C) 2014

Bassem Tossoun

ALL RIGHTS RESERVED 


\section{COMMITTEE MEMBERSHIP}

TITLE:

AUTHOR:

DATE SUBMITTED:

COMMITTEE CHAIR:

COMMITTEE MEMBER:

Engineering

COMMITTEE MEMBER:
Hybrid Silicon Mode-Locked Laser with Improved RF Power by Impedance Matching

Bassem Tossoun

September 2014

Dennis Derickson, $\mathrm{PhD}$

Professor of Electrical Engineering

Xiaomin Jin, PhD

Associate Professor of Electrical

Samuel Agbo, $\mathrm{PhD}$

Professor of Electrical Engineering 


\begin{abstract}
Hybrid Silicon Mode-Locked Laser with Improved RF Power by Impedance Matching
\end{abstract}

\title{
Bassem Tossoun
}

The mode-locked laser diode (MLLD) finds a lot of use in applications such as ultra high-speed data processing and sampling, large-capacity optical fiber communications based on optical time-division multiplexing (OTDM) systems. Integrating mode-locked lasers on silicon makes way for highly integrated silicon based photonic communication devices. The mode-locked laser being used in this thesis was built with Hybrid Silicon technology. This technology, developed by UC Santa Barbara in 2006, introduced the idea of wafer bonding a crystalline III-V layer to a Silicon-on-insulator (SOI) substrate, making integrated lasers in silicon chips possible.

Furthermore, all mode-locked lasers produce phase noise, which can be a limiting factor in the performance of optical communication systems, specifically at higher bit rates. In this thesis, we design and discuss an impedance matching solution for a hybrid silicon mode-locked laser diode to lower phase noise and reduce the drive power requirements of the device. In order to develop an impedance matching solution, a thorough measurement and analysis of the impedance of the MLLD is necessary and was carried out. Then, a narrowband solution of two $0.1 \mathrm{pF}$ chip capacitors in parallel is considered and examined as an impedance matching network for an operating frequency of $20 \mathrm{GHz}$. The hybrid silicon laser was packaged together in a module including the impedance-matching circuit for efficient RF injection.

In conclusion, a $6 \mathrm{~dB}$ reduction of power required to drive the laser diode, as well as approximately a $10 \mathrm{~dB}$ phase noise improvement, was measured with the narrow-band solution. Also, looking ahead to possible future work, we discuss a step recovery diode (SRD) driven impulse generator, which wave-shapes the RF drive to achieve efficient injection. This novel technique takes into account the time varying impedance of the absorber as the optical pulse passes through it, to provide optimum pulse shaping. 


\section{ACKNOWLEDGMENTS}

This thesis could not have been done without the help of several people. Firstly, I would like to thank my advisor, Dr. Dennis Derickson, for not only the abundant support, but for getting in me in contact with Dr. Bowers at UC Santa Barbara. On that note, I wanted to also thank Dr. John Bowers for agreeing to this collaboration project with his group, and for providing the support and access to the resources to complete this project. Sudharsanan Srinivasan has been my mentor and guide throughout this project, and has taught me so much that is so invaluable to me.

I would also like to express gratitude for the rest of my thesis committee, including Dr. Xiaomin Jin and Dr. Samuel Agbo.

Thanks to Dr. Prakash Bhartia and Ashvin Patel from Natel for helping with the ribbon bonding of the chip capacitors to the ceramic waveguides used in the project. And thanks to Aeroflex for providing us with the chip capacitors that we used for the impedance matching.

Finally, I would like to thank my parents Milad and Iman Tossoun, as well as my brother, Nader Tossoun, for all of the support throughout these last 5 years. You serve as motivation for myself to do better everyday. 


\section{TABLE OF CONTENTS}

LIST OF TABLES .............................................................................. vii

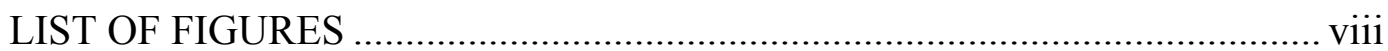

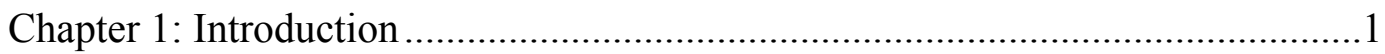

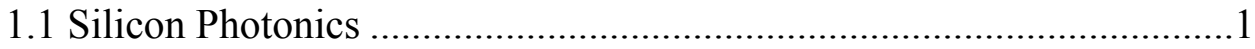

1.2 Hybrid Silicon Technology ...................................................... 2

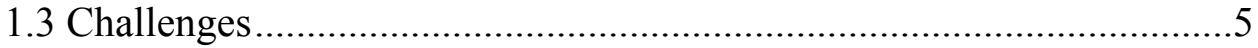

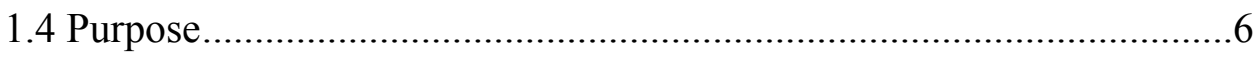

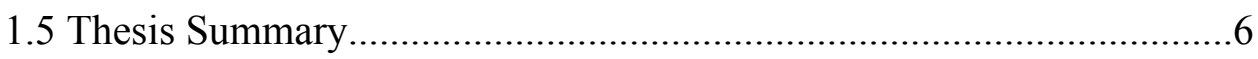

Chapter 2: Hybrid Silicon Mode-Locked Lasers ...................................................8

2.1 Lasers: Operating Principles and Physics ......................................... 8

2.1.1 Absorption and Emission .....................................................

2.2 Mode-Locked Lasers ................................................................... 10

Chapter 3: Reducing Drive Power Requirements by Impedance Matching ..........14

3.1 Impedance Matching.................................................................... 14

3.2 Test Setup for Characterizing Impedance of Laser Diode...................16

3.3 Measurements and Characterization of Diode ..................................21

3.4 Solution to Mismatched Impedance Problem ....................................30

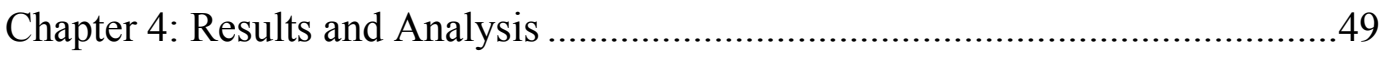

4.1 Test Setup and Measurements .....................................................49

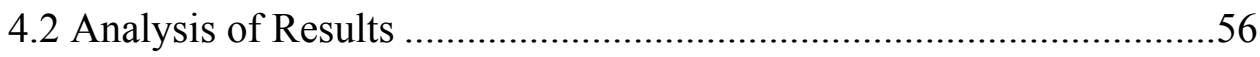

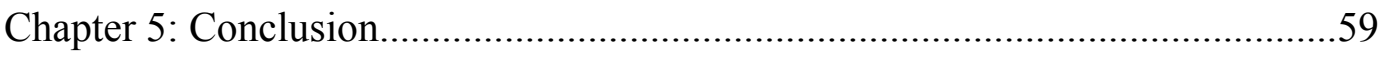

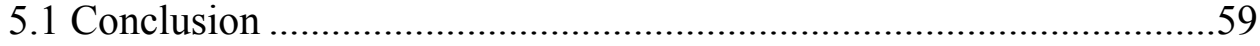

5.2 Future Work ..........................................................................61

5.2.1 “Active Matching" ..........................................................61

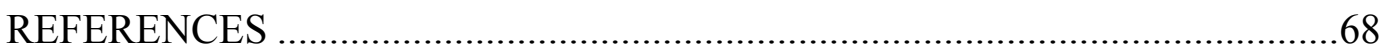




\section{LIST OF TABLES}

Table 3-1: Impedance of absorber under different biases at $20 \mathrm{GHz}$.................24

Table 3-2: Impedance Measured for Different Frequencies and Bias Conditions at which the laser is mode-locking..............................................................28 


\section{LIST OF FIGURES}

Figure 1-1: Schematic of the oxygen plasma-assisted wafer bonding process flow between the III-V layer and the SOI layer [1] ......

Figure 2-1: a) The various longitudinal modes in a Fabry-Perot cavity, b) modes in the cavity for spontaneously emitted photons and c) the lasing modes within the cavity [4]. $\mathrm{L}$ is the length of the cavity and $\lambda$ is the wavelength of the modes within the cavity.

Figure 2-2: Pulse Train Generated by Mode-Locking. The phases of each longitudinal mode $\mathrm{E}_{\mathrm{A}}, \mathrm{E}_{\mathrm{B}}$, and $\mathrm{E}_{\mathrm{C}}$ are locked together so that every $2 \mathrm{~L} / \mathrm{c}$ seconds a narrow intense pulse is formed from the superposition of the separate modes. $\mathrm{L}$ is the length of the cavity, and $\mathrm{c}$ is the speed of light

Figure 2-3: The locking of longitudinal cavity modes leads to ultrafast pulses. The pulses then circulate the cavity and grow narrower and more intense after each round trip, until reaching a saturation point

Figure 2-4: Schematic cross-section of the gain section identifying the different layers with the optical mode overlaid [11]

Figure 2-5: Representation of the gain, absorber and waveguide sections inside the laser cavity. SA is the saturable absorber and the SOAs are the semiconductor optical amplifiers [11]

Figure 3-1: Reflections caused by a mismatch in load impedance, $\mathrm{Z}_{\mathrm{L}}$, and source impedance, $\mathrm{Z}_{\mathrm{O}} \cdot \mathrm{V}^{+}$is the forward voltage, and $\mathrm{V}^{-}$is the reflected backward voltage. $\Gamma$ is the reflection coefficient, which is a measure used to determine how much of the signal is reflected by the load.

Figure 3-2: D64L and D64R are metal contact pads for the semiconductor optical amplifiers, and the contact pad D64C is for the saturable absorber. The chip has a 750 micron thickness

Figure 3-3: The substrate material used for the coplanar waveguide was Aluminum Nitride. Its dimensions were $19.65 \mathrm{~mm} \times 3 \mathrm{~mm} \times 0.76 \mathrm{~mm}$. $\varepsilon$, the dielectric constant of the ceramic material is 9.14. Then titanium-tungsten, used as ohmic contacts, and nickel, were used to stick gold on top and on bottom of the ceramic to act as conductors....

Figure 3-4: The waveguides on the Aluminum Nitride ceramic are used as $50 \Omega$ transmission lines between the various voltage and current sources connected to the laser diode. The coplanar waveguides on the left and right hand side of the ceramic are used to transmit a DC current bias from a current source to the Semiconductor Optical Amplifiers (SOAs), which act as the gain sections of the laser cavity. The coplanar waveguide in the center is used to transmit power coming from the DC voltage bias and the $20 \mathrm{GHz} \mathrm{RF}$ signal to the saturable absorber. 
Figure 3-5: Process flow diagram of die and ceramic attaching processes

Figure 3-6: The Completed Packaged MLLD Module

Figure 3-7: Layout and Measurements of Wiltron K104F Microstrip to K Female Flange Mount Connector. Through this package, the coplanar waveguide and the $\mathrm{K}$ connector would be connected. Solder would be applied into a hole on top of and onto the glass bead in between the $\mathrm{K}$ connector and coplanar waveguide for mechanical support of the $\mathrm{K}$ connector.

Figure 3-8: Diagram of test setup. The mode-locked laser diode (MLLD) is wire bonded to the ceramic waveguide and placed on a stage. The waveguide is then probed and connected to a network analyzer in order to take measurements.

Figure 3-9: S11 Frequency response when SOA is off under different SA bias conditions. The laser is not mode-locking......

Figure 3-10: S11 response (0 to $67 \mathrm{GHz}$ sweep) for when the SOAs are off and under varying saturable absorber bias conditions. The laser is not modelocking.

Figure 3-11: Impedance (real) of saturable absorber at $20 \mathrm{GHz}$. The laser is not mode-locking

Figure 3-12: Impedance (imaginary) of saturable absorber at $20 \mathrm{GHz}$. The laser is not mode-locking.

Figure 3-13: S11 Data for Laser when SOA is off (blue) and on (other colors) and biased between $149 \mathrm{~mA}$ and $245 \mathrm{~mA}$, at currents where the laser modelocks. High frequency noise can be disregarded, as the network analyzer was calibrated only up to $40 \mathrm{GHz}$.

Figure 3-14: S11 Data for Laser when both SOA segments are on and biased at $141 \mathrm{~mA}, 165 \mathrm{~mA}$, and $195 \mathrm{~mA}$. SA is biased at $-0.9 \mathrm{~V}$. Laser is mode-locking....27

Figure 3-15: S11 Data with the saturable absorber biased at $-1.1 \mathrm{~V}$, and the SOA segments biased at $141 \mathrm{~mA}, 165 \mathrm{~mA}$, and $199 \mathrm{~mA}$. Laser is mode-

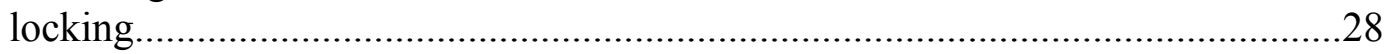

Figure 3-16: Impedance of saturable absorber when laser is mode-locking ........29

Figure 3-17: Schematic layout of open circuit stub on coplanar waveguide..........30

Figure 3-18: Lumped element Single Open Stub Matching Solution. Consists of coplanar waveguide acting as a series $\mathrm{L}$, and a capacitor shunted in parallel...31

Figure 3-19: Material Parameters for Aluminum Nitride Coplanar Waveguide ...32

Figure 3-20: Device Model of Single Open Stub Matching Network using a Coplanar Waveguide. 
Figure 3-21: Device Model of Single Open Stub Matching Network using Lumped Elements

Figure 3-22: Smith Chart and S11 Simulations of Single Open Stub Matching

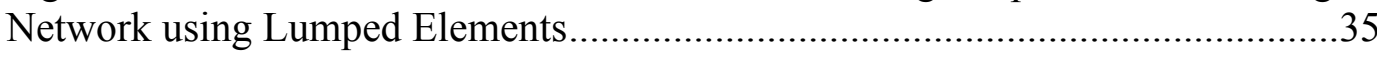

Figure 3-23: Device Model of Matching Network with Wire Bonds ......................37

Figure 3-24: Photograph of Matching Network with Wire Bonded Capacitors....37

Figure 3-25: Schematic of Test Setup for Matching Network Verification ...........38

Figure 3-26: Agilent N4373C $67 \mathrm{GHz}$ Lightwave Component Analyzer used to take RF measurements of the waveguide with matching network attached ...........39

Figure 3-27: Scattering Parameter Measurements of waveguide with ribbon bonded capacitors attached. Calibration adds a positive offset in the magnitude scale, and therefore, the magnitudes measured are not absolute

Figure 3-28: Scattering Parameter Measurements of waveguide with ribbon bonded capacitors attached. Blue arrows refer to the resonant frequencies of the LC branches of the matching circuit.

Figure 3-29: Simulated S11 (red) and S12 (blue) Measurements with Blue Arrows Pointing out dips at Resonant Frequencies of LC branches of matching Circuit

Figure 3-30: Device Model of Test Setup in order to Simulate Test Results

Figure 3-31: S11 simulation measurements for wire-bonded matching network. Marker $\mathrm{m} 1$ shows the load impedance at $20 \mathrm{GHz}$, which is far from a $50 \Omega$ match.

Figure 3-32: Photo of matching capacitors ribbon bonded to coplanar waveguide

Figure 3-33: Close-up of Gold Ribbon Bonds ......

Figure 3-34: Device Model of matching network with ribbon bonded capacitors

Figure 3-35: S11 simulation measurements for matching network with ribbon bonded capacitors

Figure 4-1: Schematic of the test setup used to measure S11 of laser.....

Figure 4-2: The impedance of the diode now is $59+\mathrm{j} 38.3 \Omega$, which is much closer to a $50 \Omega$ match than was the initial load impedance of $30-\mathrm{j} 46.4 \Omega$ 
Figure 4-3: Schematic of the test setup used to maximize coupling to lensed fiber

Figure 4-4: Schematic of the final test setup

Figure 4-5: Frequency spectrum of the diode with ribbon bonded capacitive matching network attached and passively mode locking. Saturable absorber was biased at $-1 \mathrm{~V}$, and SOAs were biased at $177 \mathrm{~mA}$. Frequency span $=100$ $\mathrm{MHz}$, resolution bandwidth $=20 \mathrm{kHz}$.

Figure 4-6: Frequency spectrum of the diode with ribbon bonded capacitive matching network attached and actively mode locking. Saturable absorber was biased at $-1 \mathrm{~V}$, and SOAs were biased at $177 \mathrm{~mA}$. RF signal generated at 8 $\mathrm{dBm}$. Frequency span $=500 \mathrm{MHz}$, resolution bandwidth $=20 \mathrm{kHz} \ldots \ldots \ldots \ldots \ldots \ldots . .54$

Figure 4-7: Frequency spectrum of the diode with ribbon bonded capacitive matching network removed and passively mode locking. Saturable absorber was biased at $-1 \mathrm{~V}$, and SOAs were biased at $177 \mathrm{~mA}$. Frequency span $=100$ $\mathrm{MHz}$, resolution bandwidth $=20 \mathrm{kHz}$

Figure 4-8: Phase noise for the laser with ribbon bonds attached to coplanar waveguide and ribbons bond not attached to the coplanar waveguide in module.....

Figure 4-9: The output of the laser is measured with a high speed photodetector and an electrical spectrum analyzer. The detected spectrum is shown for the MLLD without the impedance matching network connected (left) and with (right) impedance matching network connected. The impedance matched case had a $6 \mathrm{~dB}$ higher spectral peak indicating more efficient coupling of the RF driving source to the saturable absorber segment. Resolution bandwidth $=1$ $\mathrm{kHz}$

Figure 4-10: Source and measured output RF powers going into and coming out of the laser. A comparison is made between the case with ribbons attached to the center waveguide and without the ribbons attached. Once again, the impedance matched case consistently had approximately a $6 \mathrm{~dB}$ higher spectral peak under different input RF signal powers .................................................58

Figure 5-1: Quantum Confined Stark Effect [17] .............................................62

Figure 5-2: The circuit schematic for the SRD driven impulse generator.............64

Figure 5-3: Simulated output voltage for the SRD driven impulse train generator 


\section{Chapter 1: Introduction}

\subsection{Silicon Photonics}

The integration of photonics and microelectronics may provide the solution to many of today's bottlenecks in communications and electronic applications. With the combination of silicon-based transistors and integrated circuits, monumental advances have been made, taking us into the Information Age in which we live in today. Lasers, too, have had a large impact on technology in playing an integral role in the fiber-optics communication networks, which serve as the backbone of the Internet today. With the development of Hybrid Silicon Lasers by UC Santa Barbara in 2006, the transistor and the laser, two of some of the world's most pivotal inventions, may possibly be integrated together on one chip [1]. This opens up many new possibilities such as integrating the communications capabilities of light with the well-advanced CMOS processing of silicon wafers. Silicon valley giants such as Intel, IBM and Hewlett Packard have all been actively engaged in the field within the last decade.

On-chip optical interconnects can be a stepping-stone towards solving the bottleneck associated with copper interconnects between rack-to-rack and maybe board-to-board on a server. Metallic interconnects such as copper and aluminum are now running into problems as the industry shrinks them smaller and smaller. Copper's resistivity becomes an issue as it slows down the speed of the current up to a certain limit. This virtually acts as the largest roadblock to achieving an 
extension to Moore's Law in the future as we shrink transistors down to subnanometer scales. Also, photonic-based interconnects such as waveguides have almost loss-less efficiencies over shorter distances than copper interconnects.

With silicon photonics, optical interconnects can be made which provide higher bandwidth data transfer both between and within microchips. Developments in the field of silicon photonics such as the Hybrid Silicon Laser, Wavelength Division Multiplexing (WDM), and high-speed modulators and photo detectors, serve as a means for much higher data transmission rates. Data centers today can reap a variety of benefits by pursuing this technology, and companies like Facebook have already taken steps to establishing silicon photonics technology into their own data centers [3].

\subsection{Hybrid Silicon Technology}

In 2006, Dr. John Bowers at the University of California, Santa Barbara, had the idea of making hybrid silicon lasers by wafer bonding a crystalline IIIV layer to an Silicon-on-insulator (SOI) substrate in a similar way to silicon layers being bonded to silicon wafers to make SOI substrates [1]. By bonding the silicon to another semiconductor, which acts as a lasing medium, such as Indium Phosphide, integrated lasers in silicon chips became possible. This approach makes the most of the light-emitting properties of III-V semiconductor materials and the mature fabrication processes of silicon 
wafers to be able to fabricate on-chip silicon lasers that can be integrated with other silicon photonic devices.

Silicon manufacturing and fabrication is common in the electronic industry and is used to mass-produce low-cost electronic devices. With silicon photonics, these same manufacturing technologies are used to make low cost integrated optical devices. The hybrid silicon laser pave the way forward and revolutionizes the field of silicon photonics and future generation optical interconnects.

To describe the process with more detail, III-V functionality is heterogeneously integrated onto the SOI platform with a process called oxygen plasma assisted wafer bonding. [1] Though, the integration of the III$\mathrm{V}$ active devices with the SOI substrates requires an approach to deal with the mismatch in the different material lattice constants and thermal expansion coefficients. 


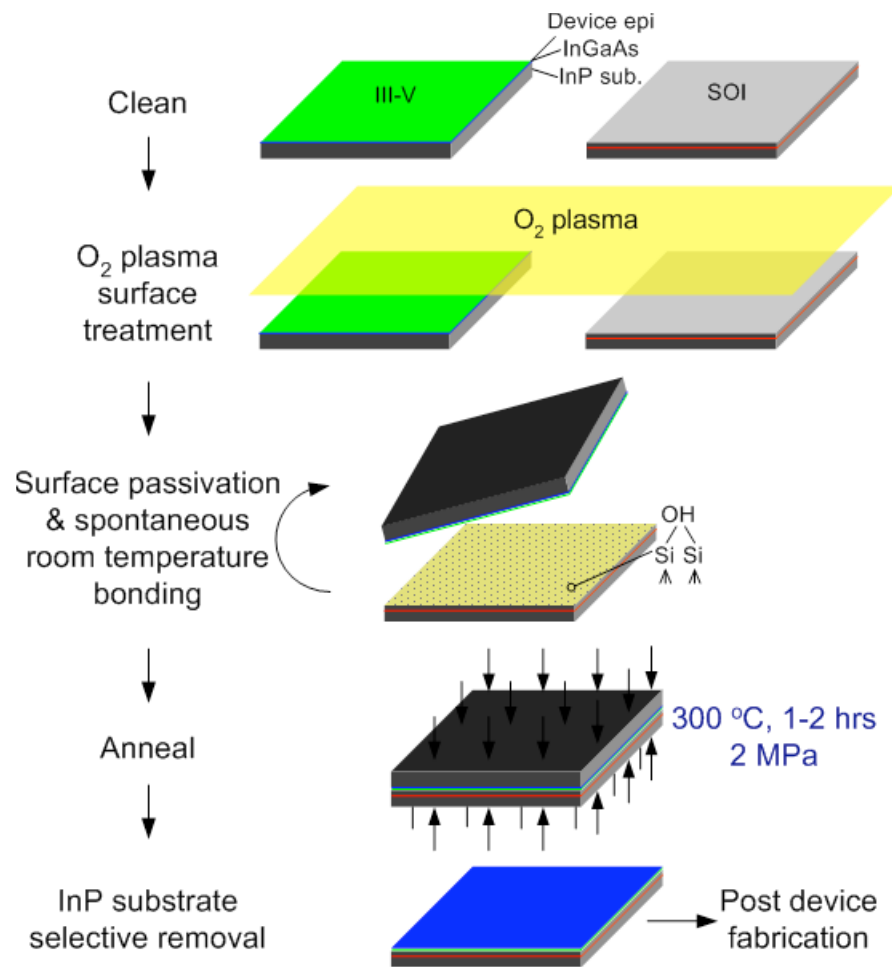

Figure 1-1: Schematic of the oxygen plasma-assisted wafer bonding process flow between the III-V layer and the SOI layer [1]

Now, photonic integrated circuits (PIC) can find medium- to largescale integration of photonic devices in silicon integrated circuits. Combining the maturity of the CMOS fabrication process with the III-V fabrication process of active and passive photonic devices bridges the gap between the optical and electrical interfaces. [6] With this, there is a large reduction in size, weight, power consumption, and cost in optical interconnects found in telecommunications systems and data centers. [7] 


\subsection{Challenges}

Photonic integrated circuits have been developed based off of mostly Indium Phosphide or Gallium Arsenide materials. Unfortunately, these materials are quite expensive in terms of mass production. Therefore, scientists have been researching ways to integrate photonics onto integrated circuits with semiconductor materials that are more affordable, such as silicon.

Despite its limits, silicon provides several properties that would actually make it a good material for diode lasers. Not only is it one of the world's most abundant materials, but, due to its simple atomic and crystal structure, it is used to make wafers of the finest purity and defect density. Other properties such as lower propagation loss than InP in the optical frequency range, large thermal conductivity and scalability also make silicon highly advantageous amongst other materials for integrated circuits.

Now, even though lasers built from silicon would be ideal, silicon is known to be a poor emitter of light. Due to its indirect bandgap, free electrons tend to recombine with holes by emitting phonons (heat) rather than light, resulting in extremely poor quantum efficiency for light emission - on the order of one photon per million electrons [1]. Because of this, it remained difficult to realize stimulated emission in silicon. 


\subsection{Purpose}

With hybrid silicon lasers, photonic circuits and devices can finally be integrated onto a silicon based platform. Hybrid silicon lasers remain the key to realizing photonics on silicon integrated circuits, and therefore, are being highly researched and improved today. This thesis promises to do just that by developing a solution to reduce electrical drive power requirements for a hybrid silicon-mode locked laser diode, making it more power-efficient.

The goal is to add to the knowledge base on hybrid silicon lasers, and to offer solutions and ideas to be used in future work and development of the hybrid silicon mode-locked laser. The characterization and data taken in this thesis can also be useful to future researchers looking for details on the electrical modulation drive of the device. This project also aims to develop a circuit that can be integrated with a mode-locked laser diode when the complete package or module is put together. Later, when a product is released, the impedance matching network developed in this thesis can be integrated into the package of the MLLD.

\subsection{Thesis Summary}

Using a Network Analyzer, the RF impedance of the saturable absorber segment of a hybrid-silicon mode-locked laser was measured. This data was used to design an impedance matching network. The impedance was then re-evaluated with the impedance matching network attached to the laser package. Finally, using an Electrical Spectrum Analyzer, the modulated optical power of a hybrid 
silicon mode-locked laser was recorded under different mode-locking bias conditions in order to evaluate performance improvements. The goal of this work is to reduce the amount of electrical RF drive power needed to actively mode-lock the laser.

Chapter 2 provides a brief overview on hybrid silicon mode-locked lasers and their theory of operation. The device structure and characteristics of the device are discussed in detail in order to provide a solid understanding of the laser. Chapter 3 discusses impedance matching in general and specifically with the mode-locked laser. An analysis of the impedance of the laser diode and how impedance matching can potentially increase the device's power efficiency is made.

Chapter 4 gives an explanation of the design of the test setup and test procedures taken to characterize the laser's RF performance. It then presents the results and provides analysis of the data. Chapter 5 makes a summary of the findings and then formulates a conclusion out of the results. Finally, it provides suggestions for future work. One area of future work is to provide optimized electrical pulse shaping waveforms for the RF drive source instead of sinusoidal drive waveforms. 


\section{Chapter 2: Hybrid Silicon Mode-Locked Lasers}

\subsection{Lasers: Operating Principles and Physics}

\subsubsection{Absorption and Emission}

Laser is an acronym for Light Amplification by Stimulated Emission of Radiation. In 1917, Einstein described the process of stimulated emission and demonstrated that the rates of absorption, spontaneous emission, and stimulated emission are related.

Coherent emission is obtained using an optical cavity [5]. The cavity uses a positive feedback mechanism to build up optical intensity. The positive feedback is provided by mirrors, or cleaved facets, placed on either side of the optical cavity, reflecting emitted light from the gain section back into the cavity. This type of system is often referred to as the Fabry-Perot resonator, and is very commonly found in laser diodes. This type of system is shown in Figure 4. To be more precise, the mirrors are actually not completely reflecting, but actually allowing some of the light to be transmitted out of the junction.

In spite of the feedback provided by the Fabry-Perot cavity, the actual output of a laser consists of a large number of discrete frequency components. These are called the axial modes or longitudinal modes of the cavity. These are bad because they can add to frequency or phase noise, which can be a limiting factor in the performance of optical communication systems. [4]

In Figure 2-1, diagrams are shown of the longitudinal modes in the laser cavity under two conditions: when light in the cavity is emitted only 
spontaneously, and when the cavity begins to lase. The broad linewidth of the spontaneous emission spectrum is eventually determined by lattice vibration effects (phonons) or from material defects/inhomogeneity.

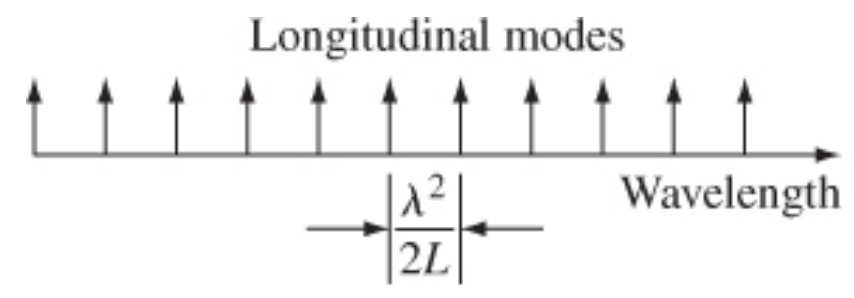

(a)

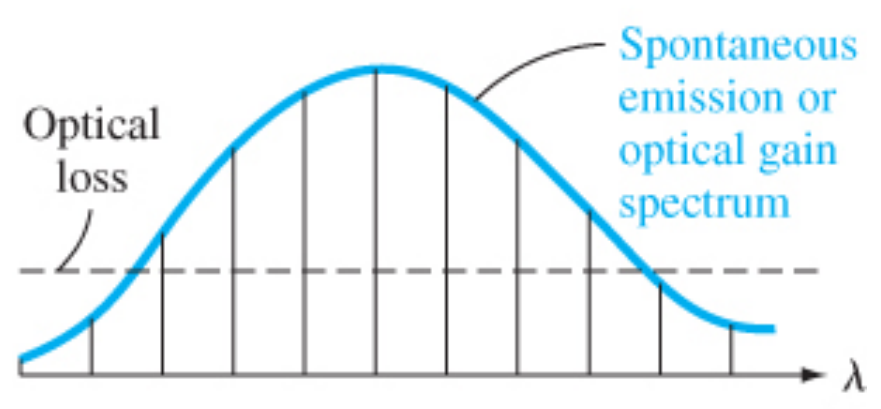

(b)

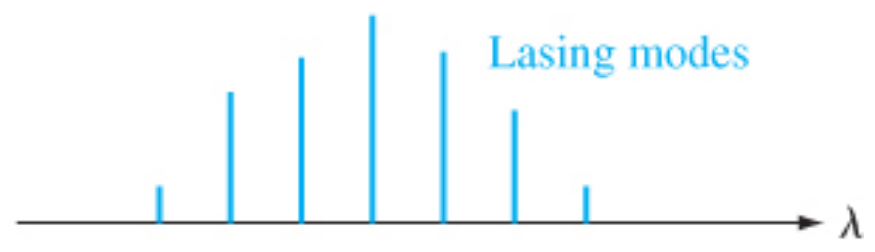

(c)

Figure 2-1: a) The various longitudinal modes in a Fabry-Perot cavity, b) modes in the cavity for spontaneously emitted photons and c) the lasing modes within the cavity [4]. $L$ is the length of the cavity and $\lambda$ is the wavelength of the modes within the cavity. 


\subsection{Mode-Locked Lasers}

Mode-locking is a technique of obtaining intense narrow pulses. Longitudinal modes within a laser cavity can oscillate with different phases. In mode locking, the phases of these different modes are locked together [5]. The modes then constitute components of a Fourier series expansion of a periodic function of period $\mathrm{T}=2 \mathrm{~L} / \mathrm{c}$ and therefore form a periodic pulse train, as shown in Figures 2-2 and 2-3.

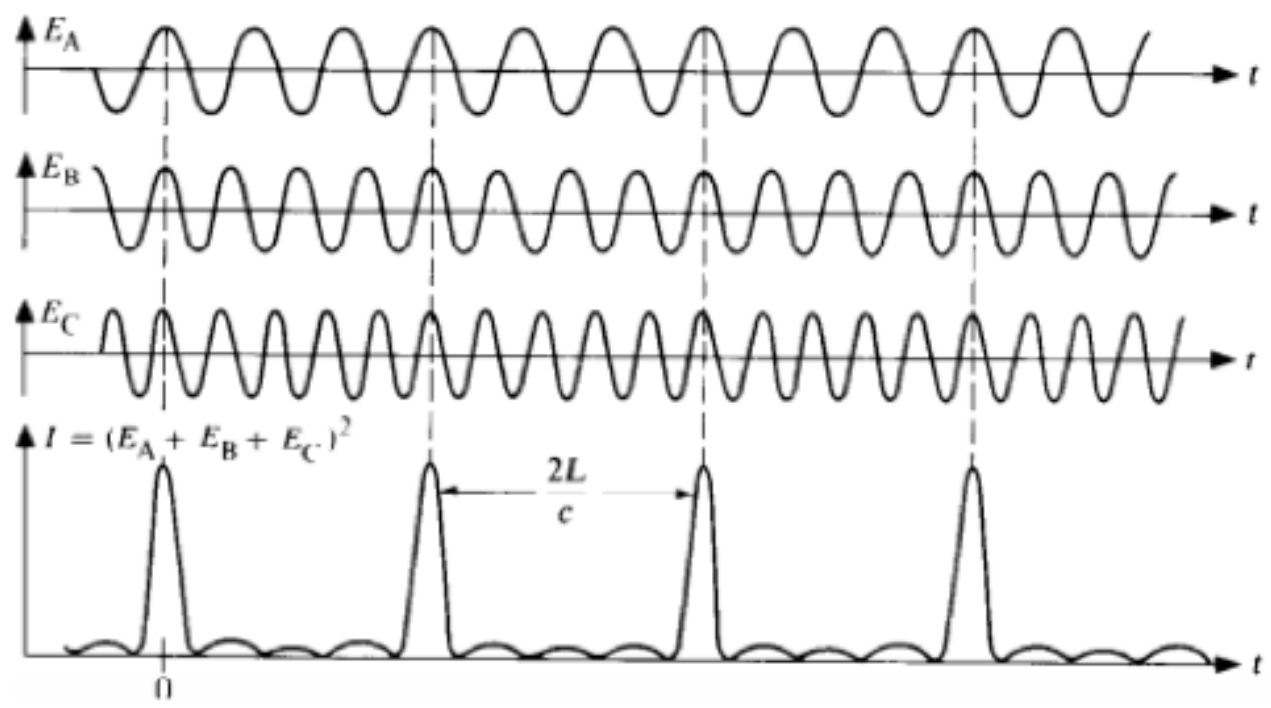

Figure 2-2: Pulse Train Generated by Mode-Locking. The phases of each longitudinal mode $\mathrm{E}_{\mathrm{A}}, \mathrm{E}_{\mathrm{B}}$, and $\mathrm{E}_{\mathrm{C}}$ are locked together so that every $2 \mathrm{~L} / \mathrm{c}$ seconds a narrow intense pulse is formed from the superposition of the separate modes. $\mathrm{L}$ is the length of the cavity, and $\mathrm{c}$ is the speed of light. 


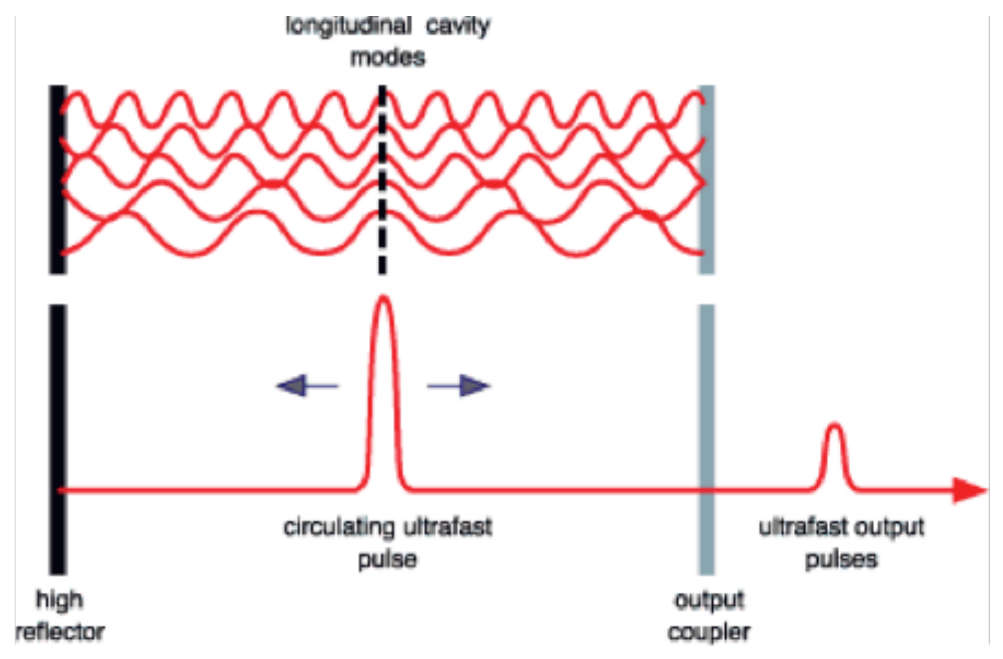

Figure 2-3: The locking of longitudinal cavity modes leads to ultrafast pulses. The pulses then circulate the cavity and grow narrower and more intense after each round trip, until reaching a saturation point.

Mode locking is achieved by incorporating a switch or an absorbing region within the laser cavity. Such a switch is called a saturable absorber. A saturable absorber suppresses low-intensity signals, and is in favor of short, intense optical pulses. Every saturable absorber has recovery time, which is a requirement for the absorption to recover between pulses. Generally, there are two types of saturable absorbers: slow and fast. The slow saturable absorber has its recovery speed longer than the optical pulse duration, so that only one side of the pulse is subject to absorption. This is the type of saturable absorber used in the laser in this thesis. Whereas, the fast saturable absorber has a recovery speed that is shorter than the optical pulse duration, and, therefore, can absorb low intensity light on both sides of the optical pulse. 
The mode-locked laser diode works under three different modes of operation. It either passively, actively mode-locks, or does a hybrid of the two. Under passive mode operation, no external modulation is used to generate pulses, and instead, the nonlinear behavior of a saturable absorber produces the optical pulses. It gives off very short pulses, but with lots of timing jitter. Current is injected into the gain sections and the absorber is reverse-biased so that it absorbs light. Under active mode operation, external modulation produces pulses that are synchronized with a given system clock. Hybrid mode operation is the combination of the passive and active mode operations.

The device being used in this thesis project is a ring-cavity mode-locked laser with an emission wavelength of $1570 \mathrm{~nm}$, which consists of a $4 \mathrm{~mm}$ long ring resonator on silicon coupled to a bus waveguide using an $85 / 15$ multi-mode interference (MMI) coupler. A $1 \mathrm{~mm}$ long hybrid silicon/III-V active section of the ring resonator provides gain. The saturable absorber is $30 \mu \mathrm{m}$ long and centered in the semiconductor optical amplifier (SOA) for stable mode locking operation [11].

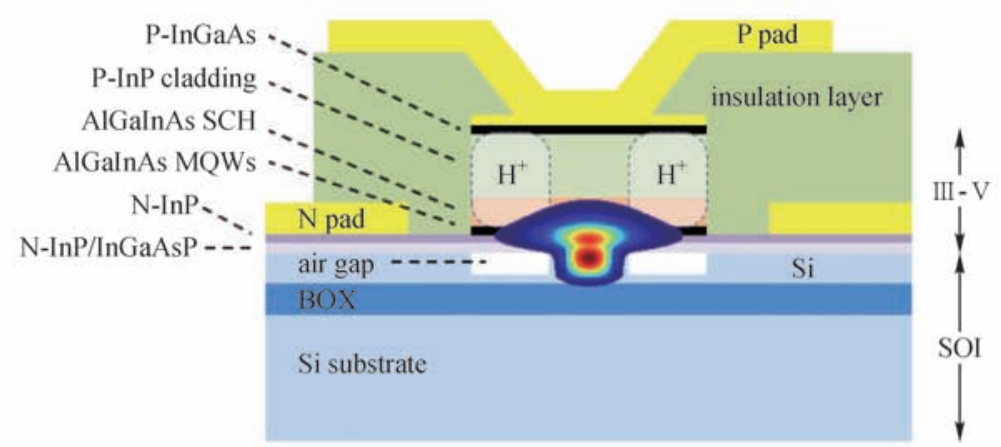

Figure 2-4: Schematic cross-section of the gain section of the laser identifying the different layers with the optical mode overlaid [11] 


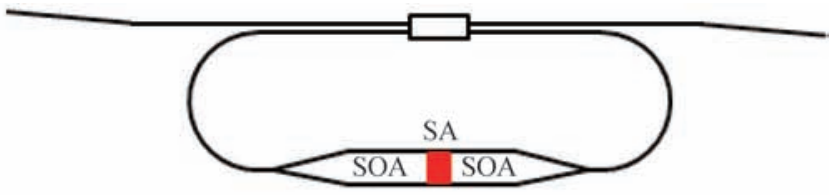

(b)

Figure 2-5: Representation of the gain, absorber and waveguide sections inside the laser cavity. SA is the saturable absorber and the SOAs are the semiconductor optical amplifiers [11] 


\section{Chapter 3: Reducing Drive Power Requirements by Impedance Matching}

\subsection{Impedance Matching}

Different materials have different propagation constants for electromagnetic waves. This mismatch in propagation constants causes reflections of electromagnetic waves when being transmitted through the different materials. For example, transmission lines all have unique characteristic impedances. The characteristic impedance of a transmission line is dependent on its shape, cladding, and type of material. When a source of power drives a signal into a load through transmission lines, reflections of the electromagnetic (EM) energy can occur. A diagram of this phenomenon is shown in Figure 3-1.

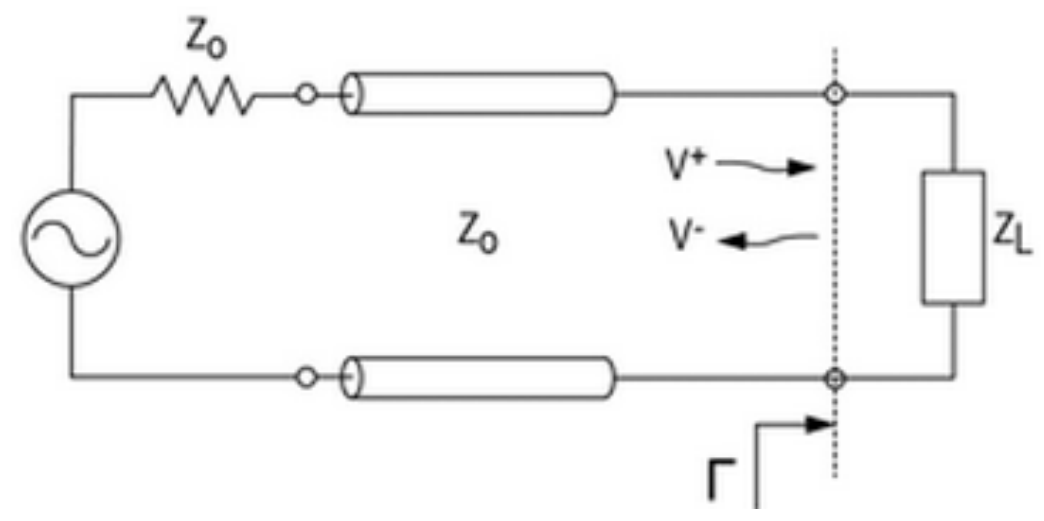

Figure 3-1: Reflections caused by a mismatch in load impedance, $\mathrm{Z}_{\mathrm{L}}$, and source impedance, $\mathrm{Z}_{\mathrm{O}} \cdot \mathrm{V}^{+}$is the forward voltage, and $\mathrm{V}^{-}$is the reflected backward voltage. $\Gamma$ is the reflection coefficient, which is a measure used to determine how much of the signal is reflected by the load.

Reflections generally mean a loss in power. In order to minimize reflection, the impedance of the load, must be equal to the complex conjugate of 
the source impedance. For two impedances to be complex conjugates their resistances must be equal, and their reactances must be equal in magnitude but of opposite signs. This way, the load impedance absorbs some of the current and leaves no reflection. This maximizes power transfer to the load, assuring that the load receives all of the EM energy coming from the source.

A strategy used to minimize reflections of EM energy and make sure that all the power coming from the source is delivered to the load is impedance matching. Impedance matching basically means adjusting the load impedance of the circuit to match the source impedance. This is normally done by adding different components into the circuit in between the source and the load, which changes the total impedance of the circuit load.

The reflection coefficient, $\Gamma$, indicates the amount of reflection experienced due to impedance mismatching. With perfect impedance matching, the reflection coefficient is zero, indicating zero reflection and maximum power transfer to the load.

$$
\Gamma=\left(Z_{L}-Z_{0}\right) /\left(Z_{L}+Z_{0}\right)
$$

$$
\begin{aligned}
& Z_{0}=\text { Source Impedance } \\
& Z_{L}=\text { Load Impedance } \\
& \qquad \text { if } Z_{L}=Z_{0} \text {, then } \Gamma=0
\end{aligned}
$$




\subsection{Test Setup for Characterizing Impedance of Laser Diode}

In this project, a $20 \mathrm{GHz}$ sinusoidal signal is sent to the saturable absorber section of a hybrid silicon mode-locked laser. Because the absorber has an impedance that is different than the source impedance of the signal generator (50 ), there needs to be a matching network that can solve this problem. With a proper impedance matching network, all of the RF power coming from the source would essentially be transferred to the saturable absorber. This assures maximum RF injection efficiency for when the laser diode is being actively mode-locked.

Figure 3-2 is a schematic of the chip interface of the laser being used. The gold bond pads on the chip interface make ohmic contacts with the different parts of the laser cavity.

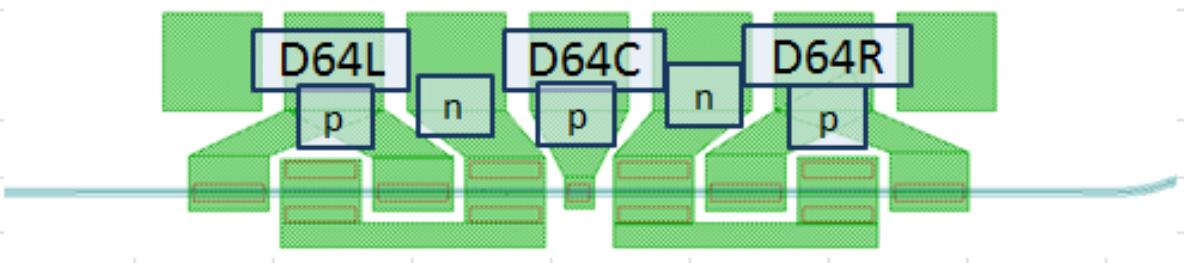

Figure 3-2: D64L and D64R are metal contact pads for the semiconductor optical amplifiers, and the contact pad D64C is for the saturable absorber. The chip has a 750 micron thickness.

In order to effectively impedance match, the load impedance, which is the saturable absorber of the mode-locked laser diode in this case, needs to be known. To achieve a good characterization of the saturable absorber' $\mathrm{s}$ impedance, the 
individual laser diode die was inserted into a package using eutectic die attaching processes for easy connection to power sources and test equipment. First, a copper block was coated with gold, and then indium was used for adhesion of the die to the block. The block is then placed on a heated stage at $195^{\circ} \mathrm{C}$ in order to bond the die to the block. Mechanical pressure is applied onto the block in order to support the bonding reaction. Using solder tape or epoxy was another option considered, but there was the possibility of it creeping up on the facet and covering the waveguide.

Next, a coplanar waveguide was inserted onto the copper block in between the signal source and the laser diode as a transmission line. Generally, in a grounded coplanar waveguide, a conductor is placed on top and bottom of a dielectric. Two slots separate three different sections of the top-placed metal, acting as waveguides for the different transverse electromagnetic (TEM) modes which propagate in the substrate. A cross section of a coplanar waveguide is shown in Figure 3-3. The center conductor is separated from the side conductors, by two slots. The two side conductors, and the conductor on the bottom of the substrate act as ground planes. The gaps, in between the conductors, act as waveguides for the TEM waves propagating in the material. The width of the gaps, as well as the width of the center conductor, are chosen to determine the characteristic impedance of the transmission line, as well as which modes may propagate through the substrate. Figure 3-4 shows the design of the coplanar waveguide that was used. 


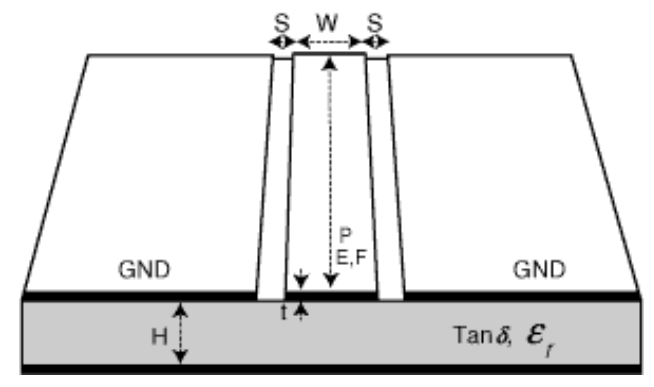

Figure 3-3: The substrate material used for the coplanar waveguide was Aluminum Nitride. Its dimensions were $19.65 \mathrm{~mm} \times 3 \mathrm{~mm} \times 0.76 \mathrm{~mm}$. $\varepsilon$, the dielectric constant of the ceramic material is 9.14 . Then titanium-tungsten, used as ohmic contacts, and nickel, were used to stick gold on top and on bottom of the ceramic to act as conductors.

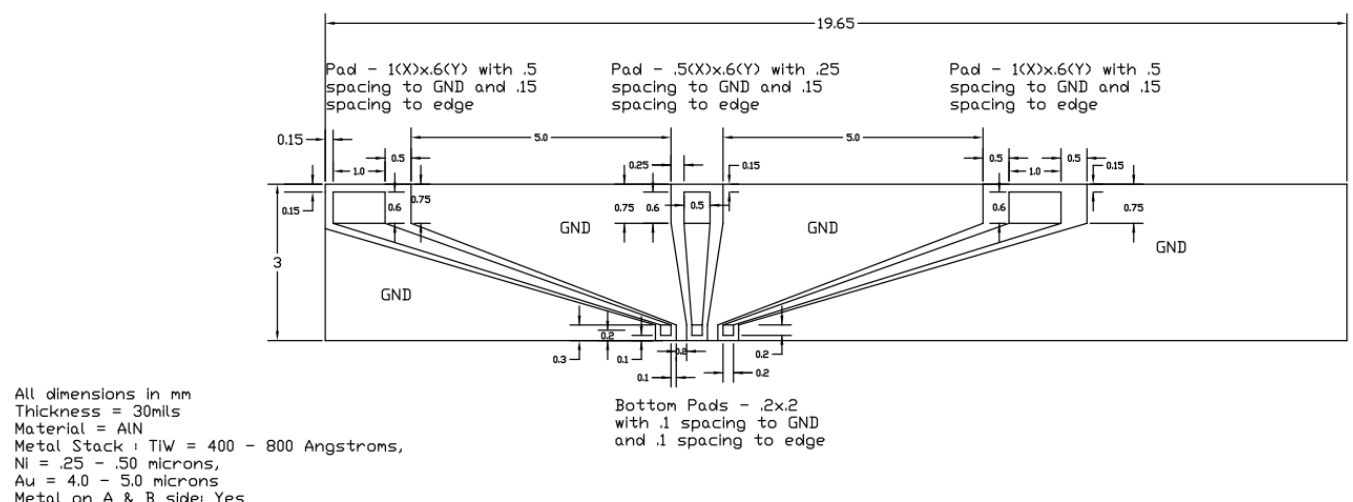

Figure 3-4: The waveguides on the Aluminum Nitride ceramic are used as $50 \Omega$ transmission lines between the various voltage and current sources connected to the laser diode. The coplanar waveguides on the left and right hand side of the ceramic are used to transmit a DC current bias from a current source to the Semiconductor Optical Amplifiers (SOAs), which act as the gain sections of the laser cavity. The coplanar waveguide in the center is used to transmit power coming from the DC voltage bias and the $20 \mathrm{GHz}$ RF signal to the saturable absorber.

The die containing the mode-locked laser diode (MLLD) is then wire bonded to the coplanar waveguides on the ceramic substrate. Figure 3-5 shows a process flow diagram of the die and ceramic attaching procedure. 


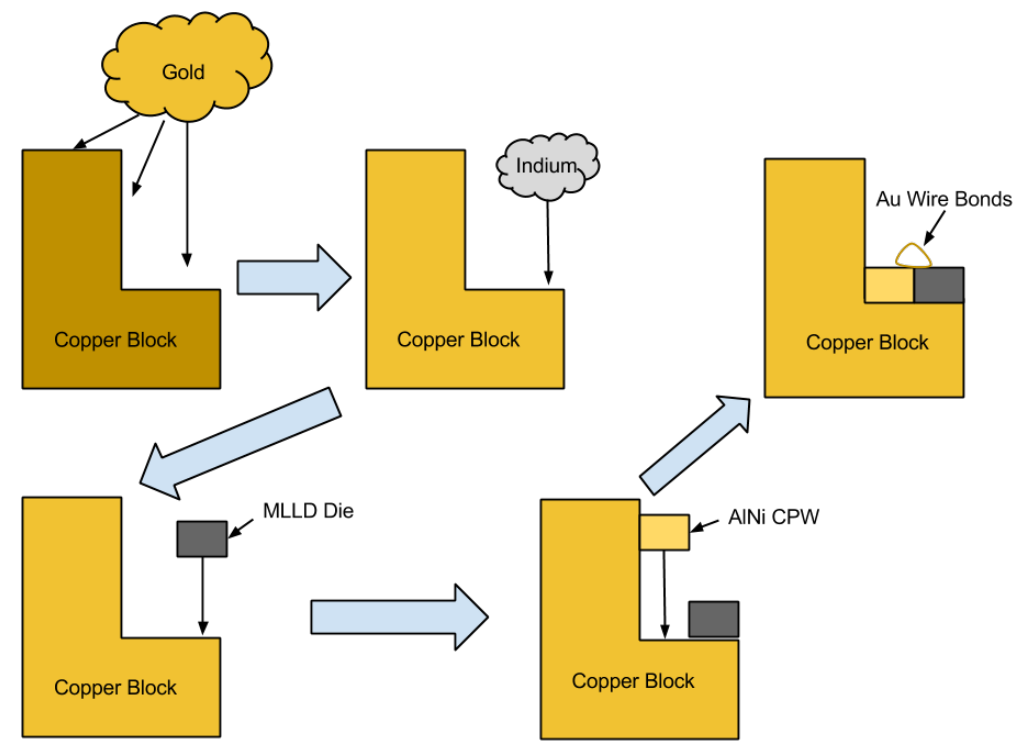

Figure 3-5: Process flow diagram of die and ceramic attaching processes

A DC bias and an RF signal is applied to the saturable absorber to hybridly mode-lock the laser. The two signals are connected together with a bias tee, which is then transferred onto the center coplanar waveguide. Ground probes are to be placed on top of the ground planes of the waveguide. Figure 3-6 is a photo of the complete package, and Figure 3-7 shows measurements and layout designs of the copper block that was used to package the diode. 


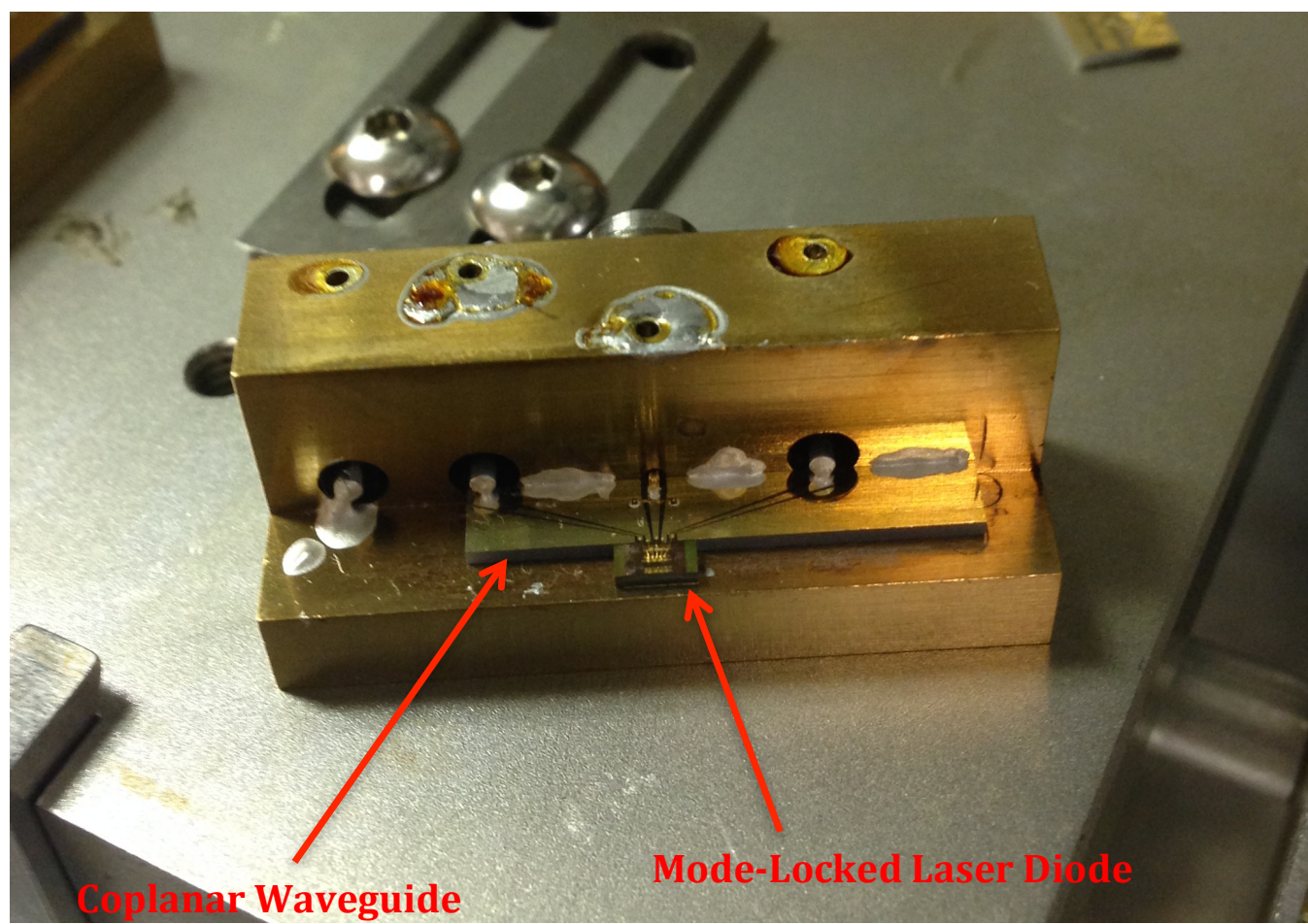

Figure 3-6: The Completed Packaged MLLD Module
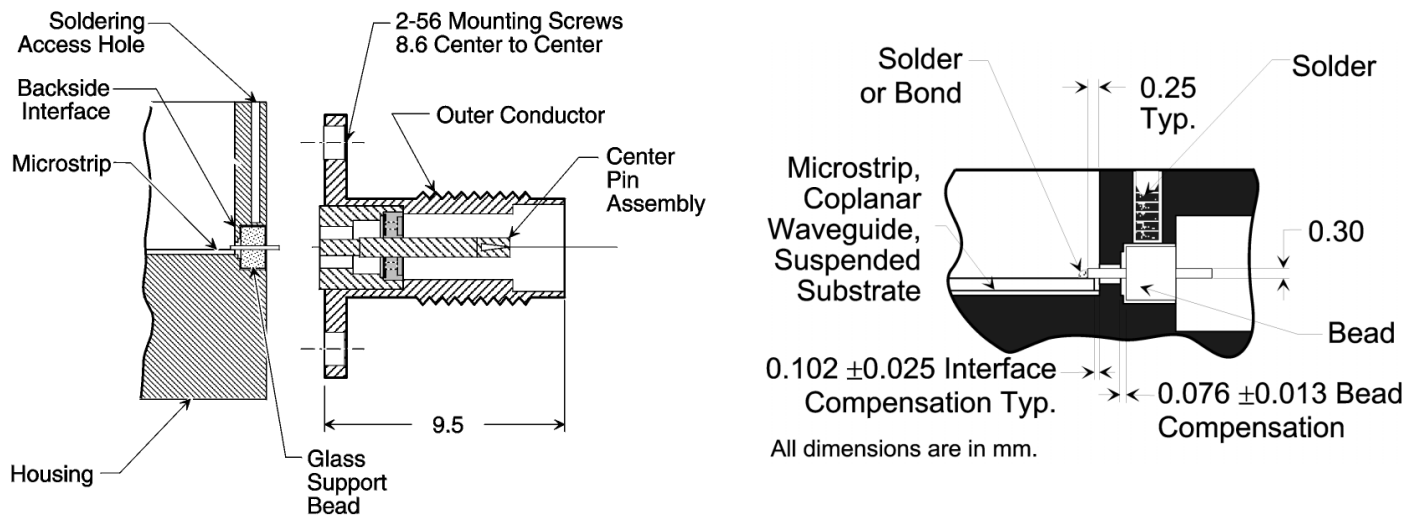

Figure 3-7: Layout and Measurements of Wiltron K104F Microstrip to K Female Flange Mount Connector. Through this package, the coplanar waveguide and the $\mathrm{K}$ connector would be connected. Solder would be applied into a hole on top of and onto the glass bead in between the $\mathrm{K}$ connector and coplanar waveguide for mechanical support of the $\mathrm{K}$ connector. 


\subsection{Measurements and Characterization of Diode}

After the MLLD was completely packaged, a high-speed network analyzer was used to measure the absorber's Scattering (S-) parameters. The network analyzer that was used was the Agilent E8361A PNA Network Analyzer. $1.5 \mathrm{~mm}$ wide-pitch RF probes were placed on top of the coplanar waveguide, and connected to the network analyzer. Measurements were taken for reflection and coefficients and S-parameters of the laser diode under several bias conditions and under a linear frequency sweep from 0 to $40 \mathrm{GHz}$. Figure 3-8 shows a schematic diagram of the test measurement setup.

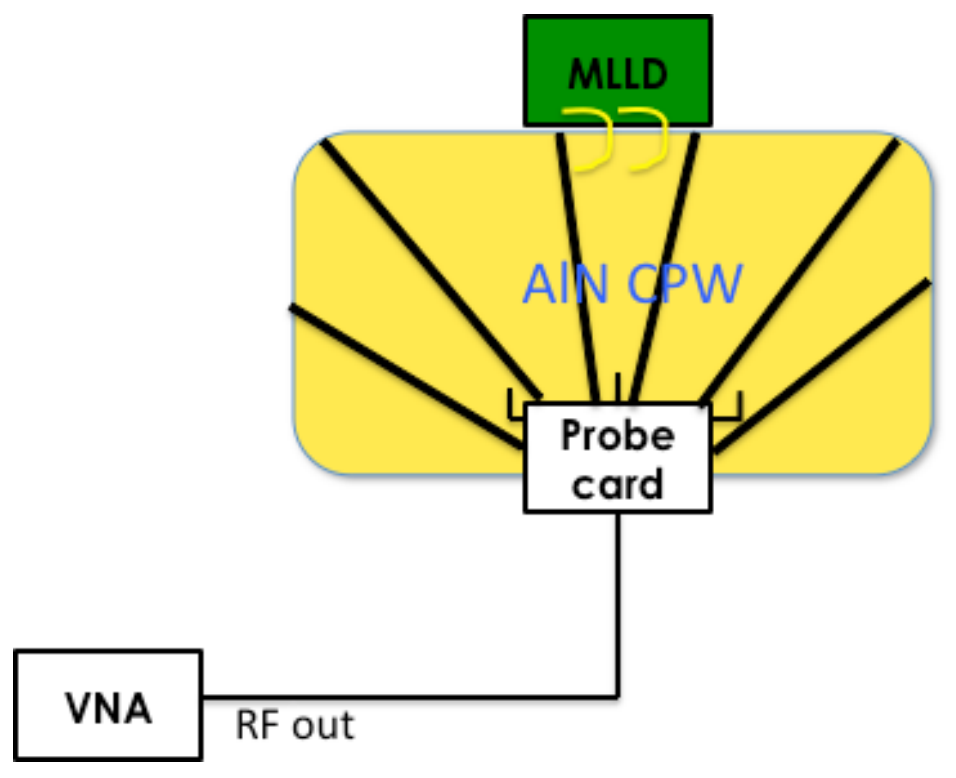

Figure 3-8: Diagram of test setup. The mode-locked laser diode (MLLD) is wire bonded to the ceramic waveguide and placed on a stage. The waveguide is then probed and connected to a network analyzer in order to take measurements. 


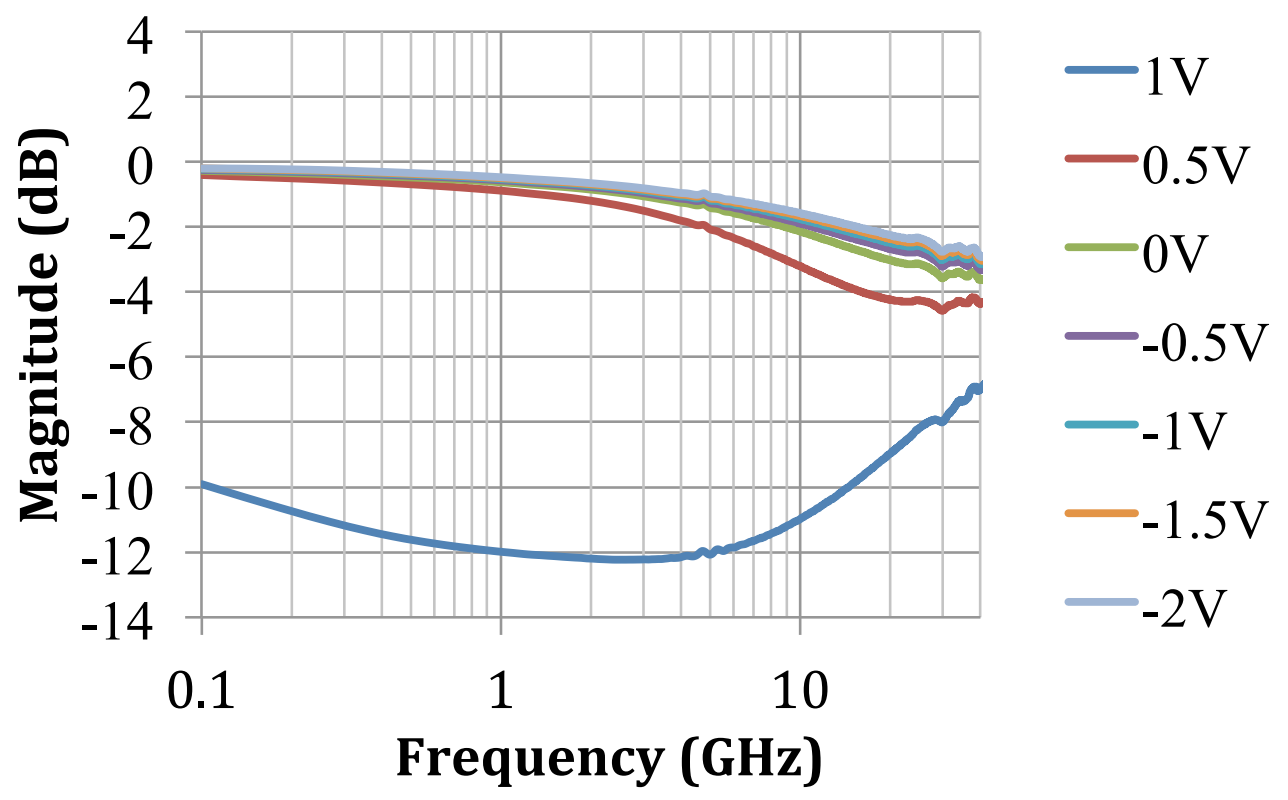

Figure 3-9: S11 Frequency response when SOA is off under different SA bias conditions. The laser is not mode-locking.

Figure 3-10 represents how the S11 parameters on a Smith Chart look for when the device is not mode-locked under different saturable absorber biases. 


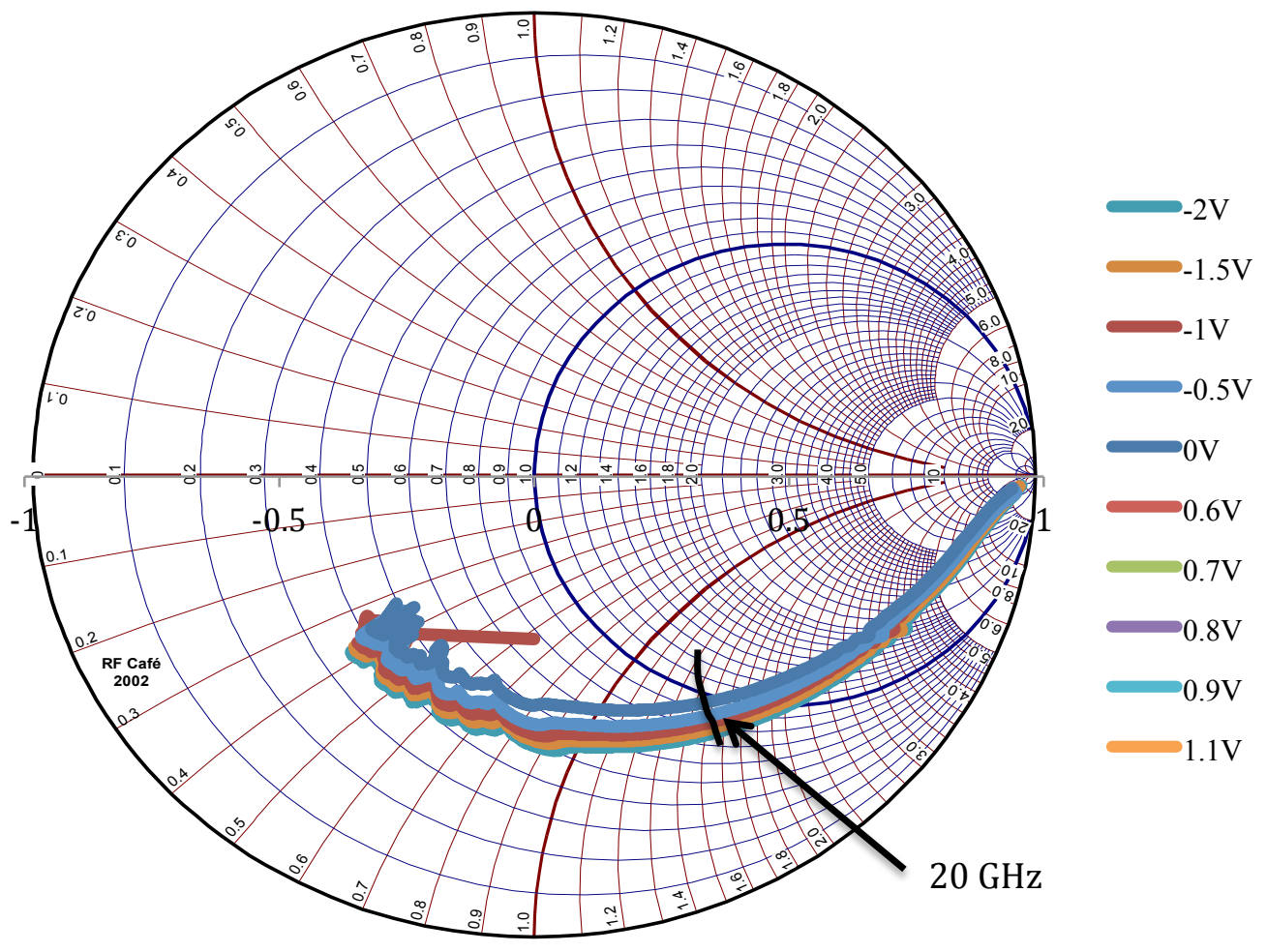

Figure 3-10: $\mathrm{S} 11$ response (0 to $67 \mathrm{GHz}$ sweep) for when the SOAs are off and under varying saturable absorber bias conditions. The laser is not mode-locking.

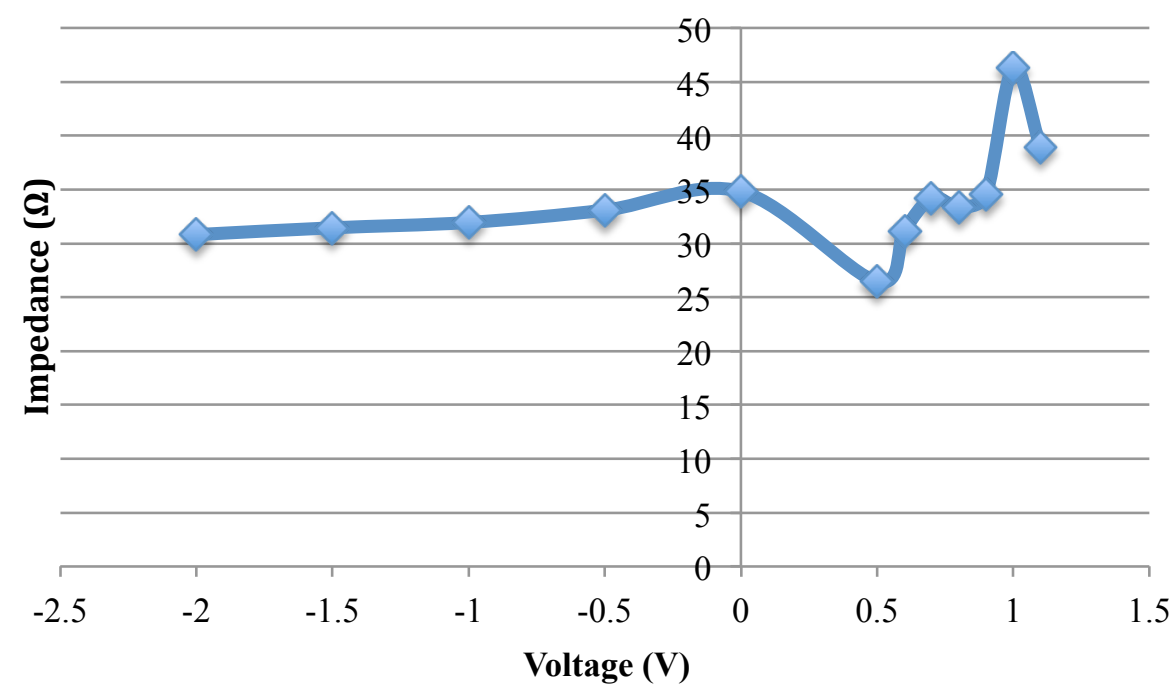

Figure 3-11: Impedance (real) of saturable absorber at $20 \mathrm{GHz}$. The laser is not mode-locking. 


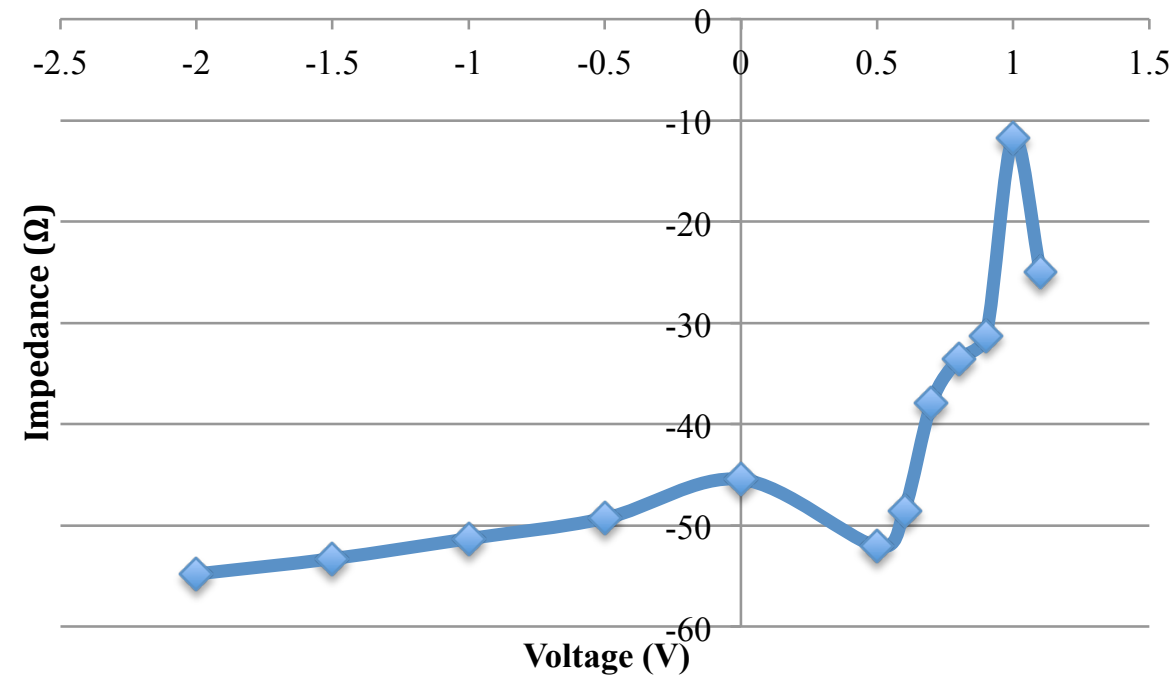

Figure 3-12: Impedance (imaginary) of saturable absorber at $20 \mathrm{GHz}$. The laser is not mode-locking.

Table 3-1: Impedance of absorber under different biases at $20 \mathrm{GHz}$

\begin{tabular}{|l|l|l|}
\hline Voltage & Zin (Real) & Zin (Imaginary) \\
\hline $\mathbf{- 2}$ & 30.82724404 & -54.78981189 \\
\hline $\mathbf{- 1 . 5}$ & 31.45158459 & -53.2761013 \\
\hline $\mathbf{- 1}$ & 31.94813318 & -51.30230386 \\
\hline $\mathbf{- 0 . 5}$ & 33.08765489 & -49.29070905 \\
\hline $\mathbf{0}$ & 34.82487923 & -45.44317588 \\
\hline $\mathbf{0 . 5}$ & 26.45608614 & -52.0552429 \\
\hline $\mathbf{0 . 6}$ & 31.1665594 & -48.50924936 \\
\hline $\mathbf{0 . 7}$ & 34.17067172 & -37.90159007 \\
\hline $\mathbf{0 . 8}$ & 33.40449297 & -33.55156359 \\
\hline
\end{tabular}




\begin{tabular}{|l|l|l|}
\hline $\mathbf{0 . 9}$ & 34.51242026 & -31.21794038 \\
\hline $\mathbf{1}$ & 46.31011065 & -11.78158602 \\
\hline $\mathbf{1 . 1}$ & 38.96639076 & -24.97435431 \\
\hline & & \\
\hline Average & $32.18497264 \Omega$ & $-\mathrm{j} 45.73376883 \Omega$ \\
\hline
\end{tabular}

The impedance of the absorber under the different biases were all averaged and an estimate of the diode was made to be 32.18497264 $\mathrm{j} 45.73376883 \Omega$. The negative imaginary number indicates that it is capacitive. (Bottom half of Smith Chart indicated Capacitive reactance.)

$$
\mathrm{Z}=1 / \mathrm{j} \omega \mathrm{C} \Rightarrow \mathrm{C}=1 /\left[\left(2 \pi^{*} 20 \mathrm{GHz}\right) *(45.73376883 \Omega)\right]=0.174 \mathrm{pF}
$$




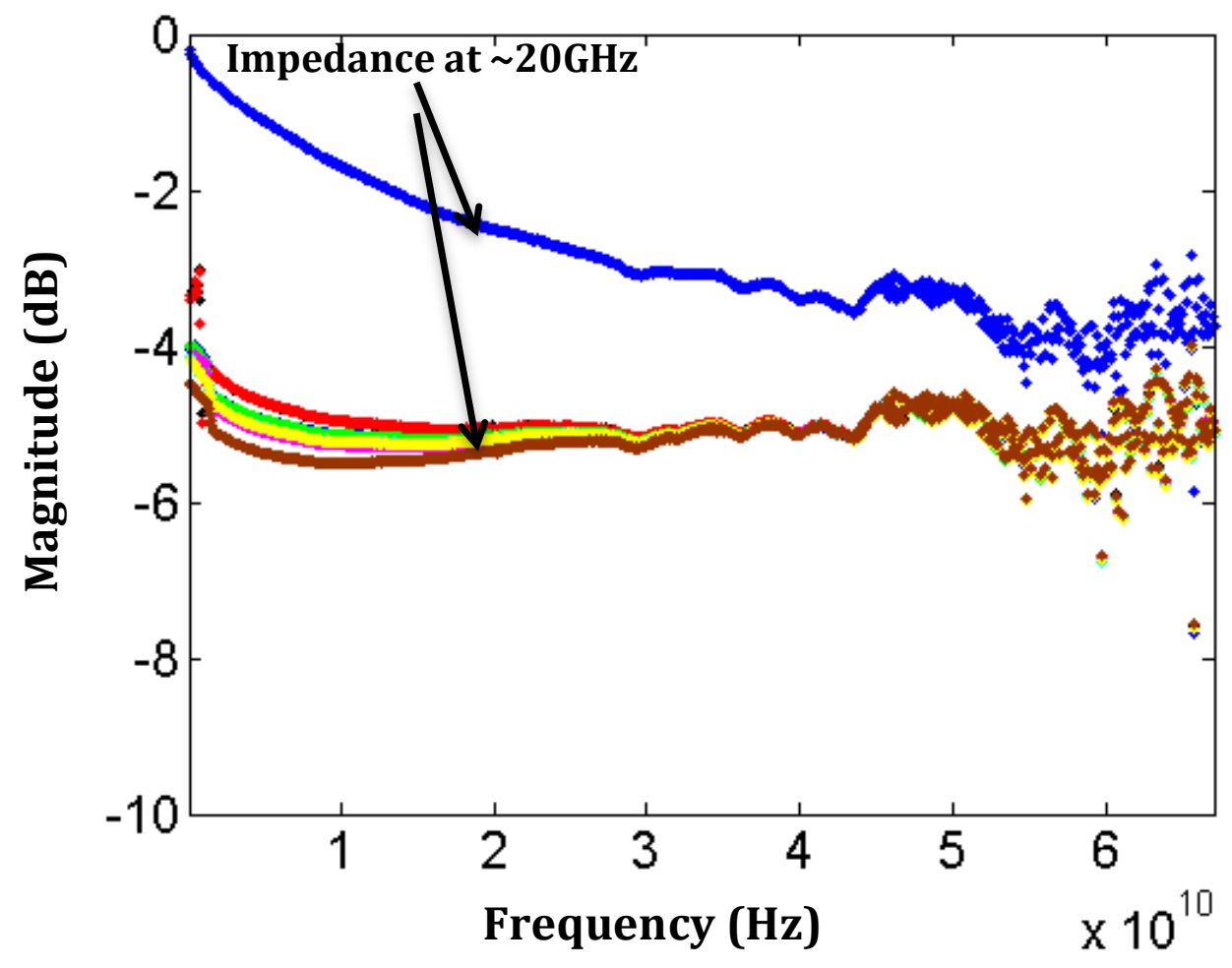

Figure 3-13: S11 Data for Laser when SOA is off (blue) and on (other colors) and biased between $149 \mathrm{~mA}$ and $245 \mathrm{~mA}$, at currents where the laser mode-locks. High frequency noise can be disregarded, as the network analyzer was calibrated only up to $40 \mathrm{GHz}$.

Figure 3-13 shows the device impedance when the laser is off and when the laser is mode-locking. The blue curve in the figure represents when the SOA is off and the absorber voltage is $-0.9 \mathrm{~V}$, and the rest of the curves represent the device when the SOA is on. There was a slight change in impedance for when the SOA is on or off. The additional impedance comes from photogenerated carriers that are generated in the active regions of the cavity when current is pumped into the SOAs. 


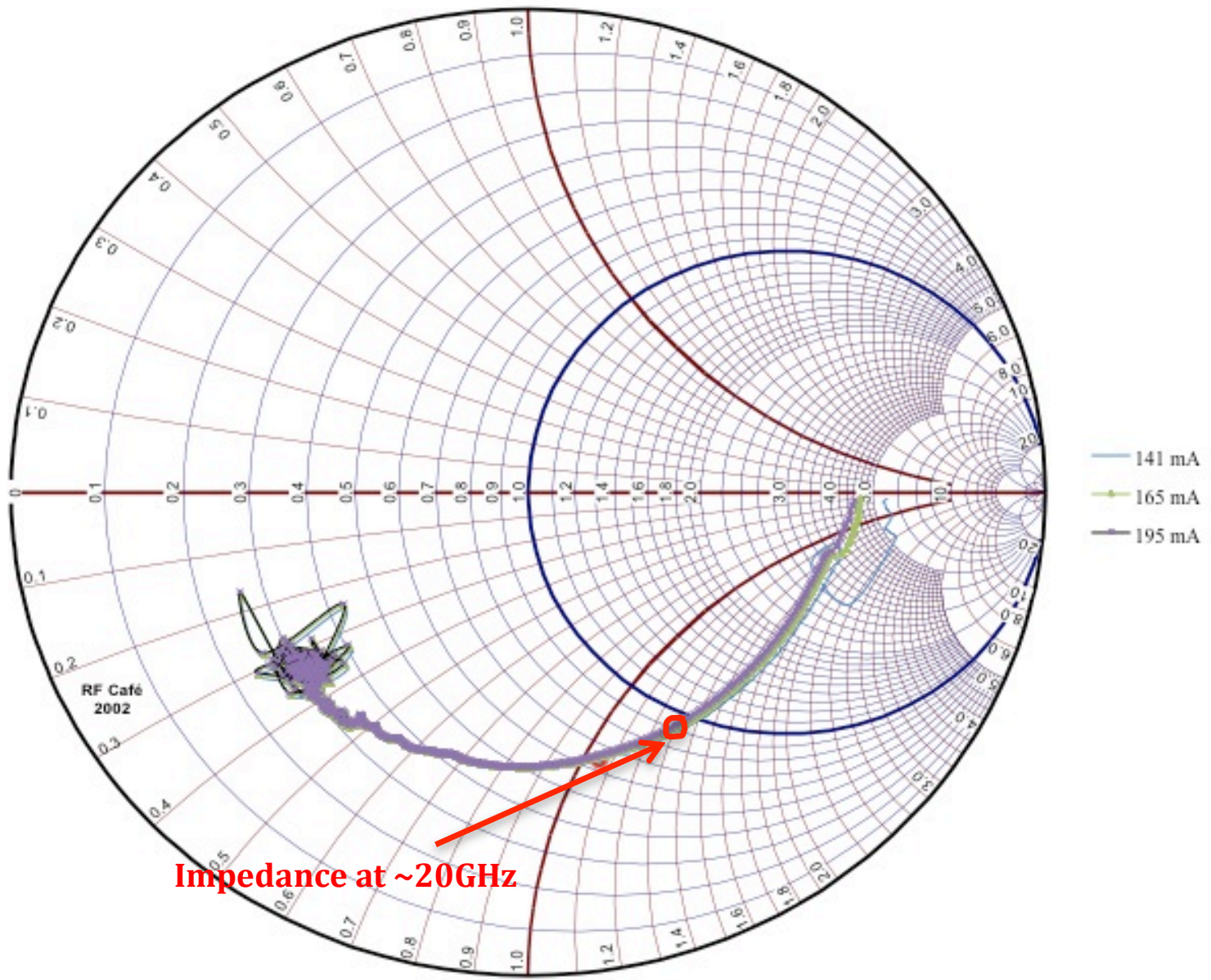

Figure 3-14: S11 Data for Laser when both SOA segments are on and biased at $141 \mathrm{~mA}, 165 \mathrm{~mA}$, and $195 \mathrm{~mA}$. SA is biased at -0.9V. Laser is mode-locking. 


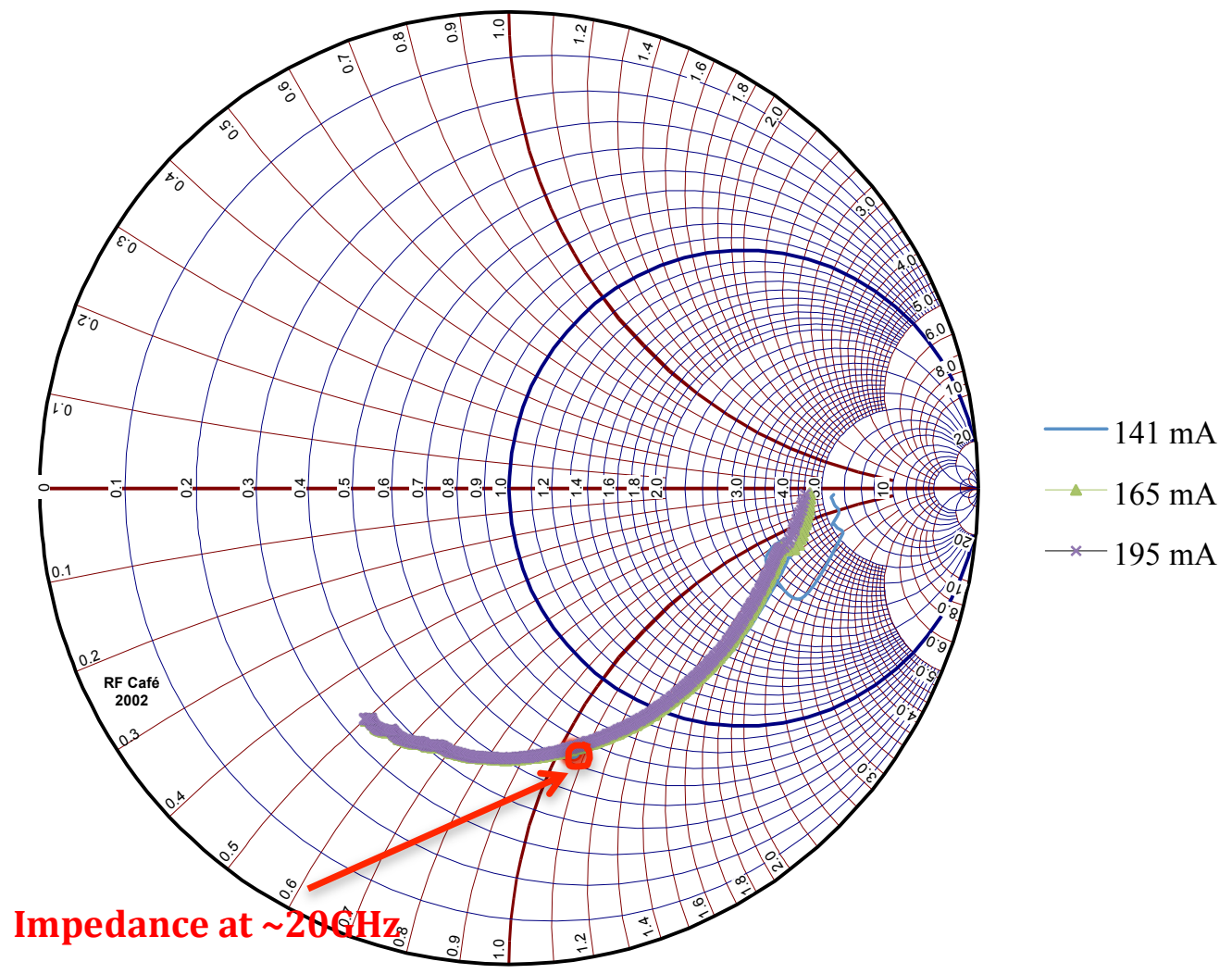

Figure 3-15: S11 Data with the saturable absorber biased at $-1.1 \mathrm{~V}$, and the SOA segments biased at $141 \mathrm{~mA}, 165 \mathrm{~mA}$, and $199 \mathrm{~mA}$. Laser is mode-locking.

Table 3-2: Impedance Measured for Different Frequencies and Bias Conditions at which the laser is mode-locking

\begin{tabular}{|l|l|l|l|l|l|}
\hline Frequency & SA Bias & SOA Biases & Impedance & $\mathrm{R}$ & $\mathrm{C}$ \\
\hline $19.919125 \mathrm{GHz}$ & $-0.9 \mathrm{~V}$ & $141 \mathrm{~mA}$ & $30.5-\mathrm{j} 48.4 \Omega$ & $30.5 \Omega$ & $0.164 \mathrm{pF}$ \\
\hline $20.00275 \mathrm{GHz}$ & $-0.9 \mathrm{~V}$ & $141 \mathrm{~mA}$ & $29.4-\mathrm{j} 47.3 \Omega$ & $29.4 \Omega$ & $0.168 \mathrm{pF}$ \\
\hline $20.17 \mathrm{GHz}$ & $-0.9 \mathrm{~V}$ & $141 \mathrm{~mA}$ & $29.3-\mathrm{j} 47.1 \Omega$ & $29.3 \Omega$ & $0.1675 \mathrm{pF}$ \\
\hline $20.17 \mathrm{GHz}$ & $-1.1 \mathrm{~V}$ & $199 \mathrm{~mA}$ & $30.2-\mathrm{j} 45.9$ & $30.2 \Omega$ & $0.172 \mathrm{pF}$ \\
\hline
\end{tabular}

Average Impedance: $\mathbf{R}=\mathbf{3 0} \Omega, \mathbf{C}=\mathbf{0 . 1 7} \mathbf{p F}$ 
Although the nonlinear behavior of a laser diode presents a frequency dependent impedance and is dependent upon the current, the impedance of the laser diode used in our case was given as approximately a series resistance of 30 Ohms plus a capacitance of 17 nanoFarads for a frequency range of $19.875 \mathrm{GHz}-$ 20.177 GHz. The matching impedance circuit now presents itself as a more reachable task with a defined load impedance and a $50 \Omega$ source impedance.

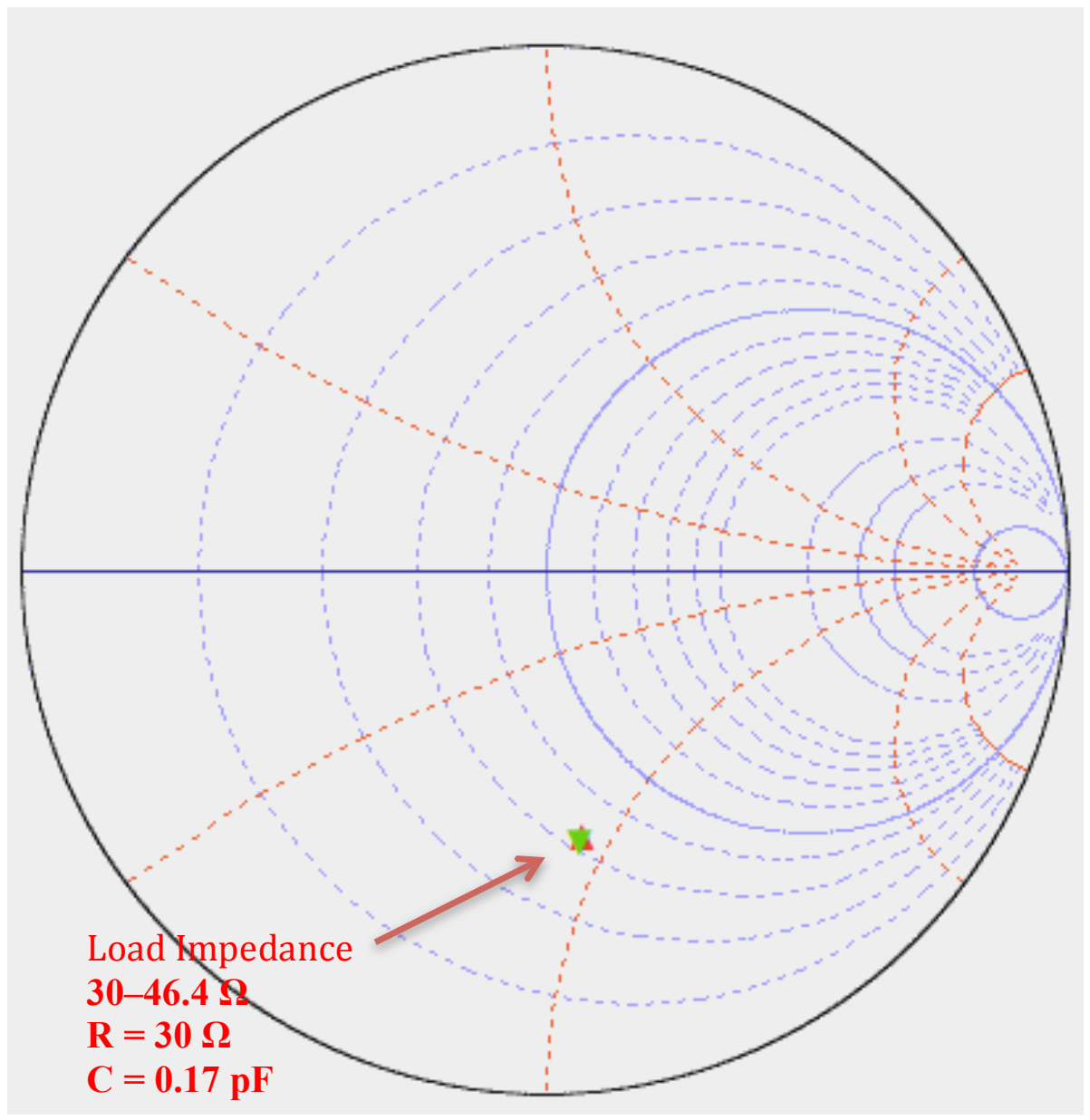

Figure 3-16: Impedance of saturable absorber when laser is mode-locking 


\subsection{Solution to Mismatched Impedance Problem}

In order to efficiently couple energy from the $50 \Omega$ source impedance of the driving RF source to the saturable absorber segment, a passive matching network was designed. Now, that there is an accurate measurement of the load impedance, a matching network solution can be made. The task of matching to the impedance of the diode is now presented.

The first suggested solution was a single open stub matching network, which would be fabricated on another coplanar waveguide. In Figure 3-18, a Smith Chart is shown for this solution. A $50 \Omega$ transmission line, representing the coplanar waveguide, would be used to transform the impedance to one with a positive reactance. Then a capacitive open stub would be used to transform the impedance to $50 \Omega$.

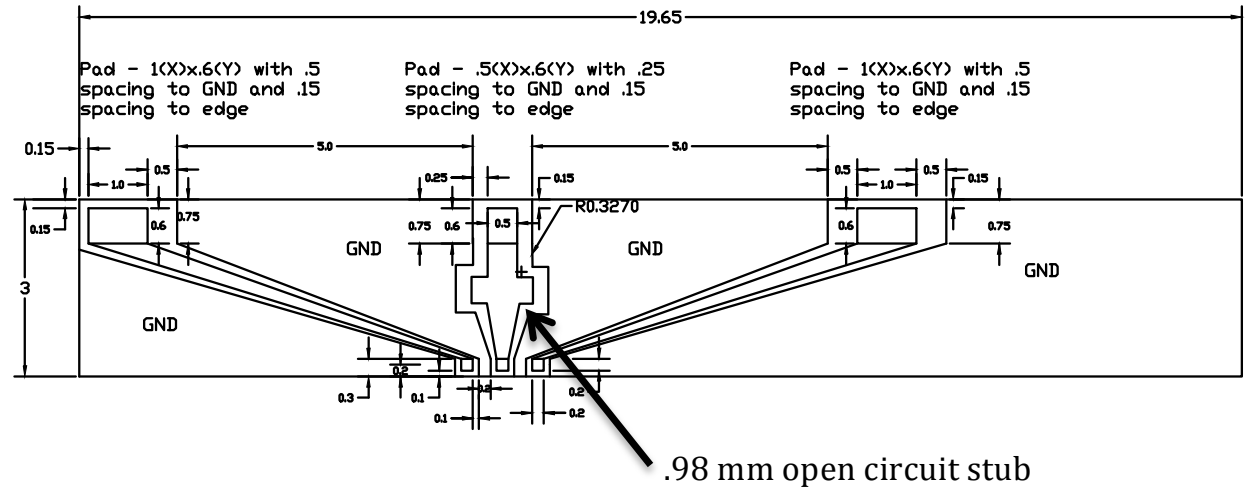

Figure 3-17: Schematic layout of open circuit stub on coplanar waveguide 


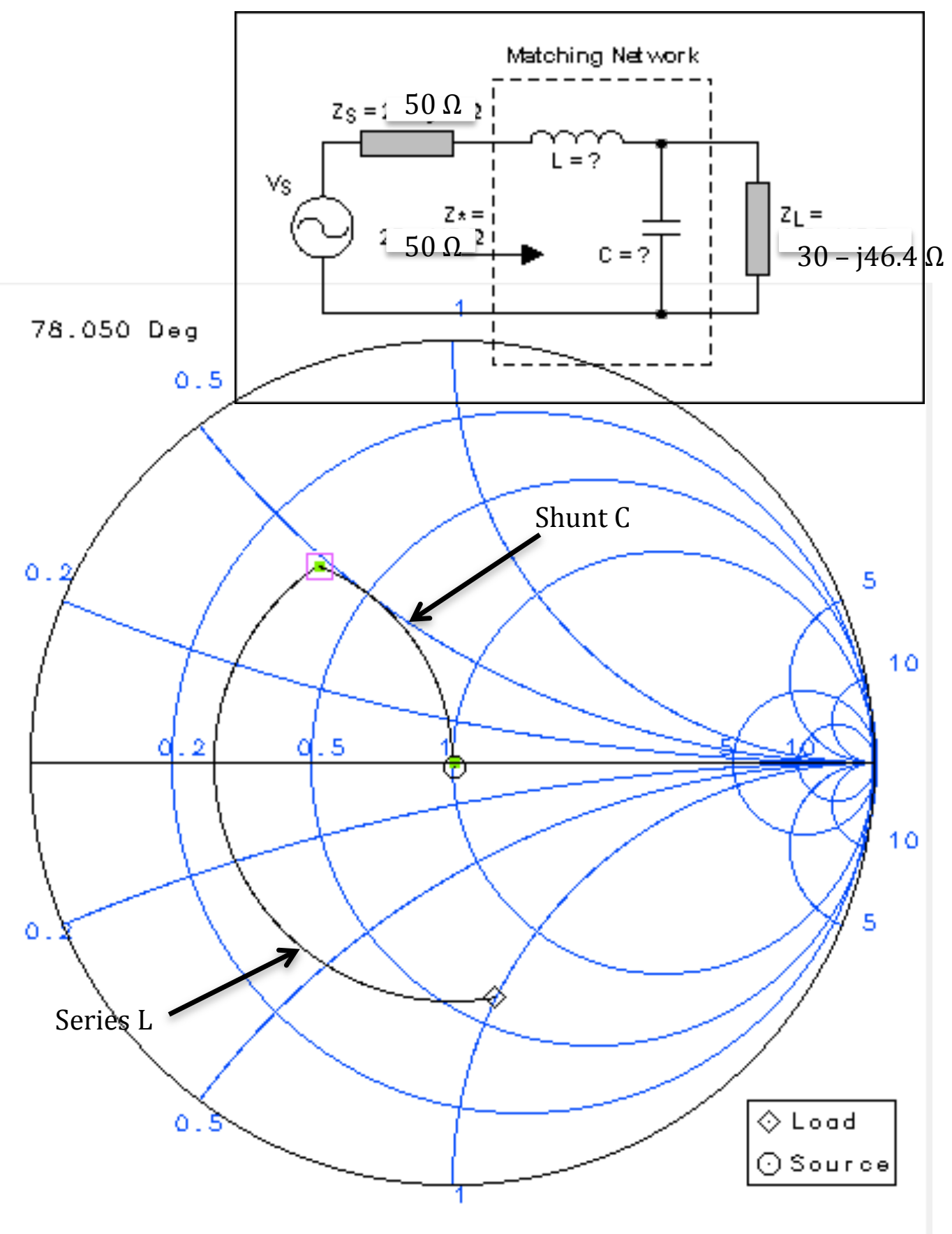

Figure 3-18: Lumped element Single Open Stub Matching Solution. Consists of coplanar waveguide acting as a series L, and a capacitor shunted in parallel.

First, the length of the coplanar waveguide that would be used as transmission line in between the load and the open stub was calculated. Using the LineCalc tool in Agilent Advanced Design System (ADS), the material's 
parameters were put into the software, and an effective dielectric constant of permittivity was given for Aluminum Nitride. This then was used to calculate the phase velocity of light in the medium. Afterwards, the wavelength of light was calculated for the propagation of light in the material, and from there the effective length of the transmission line was calculated. The calculations are shown in Figure 3-19:

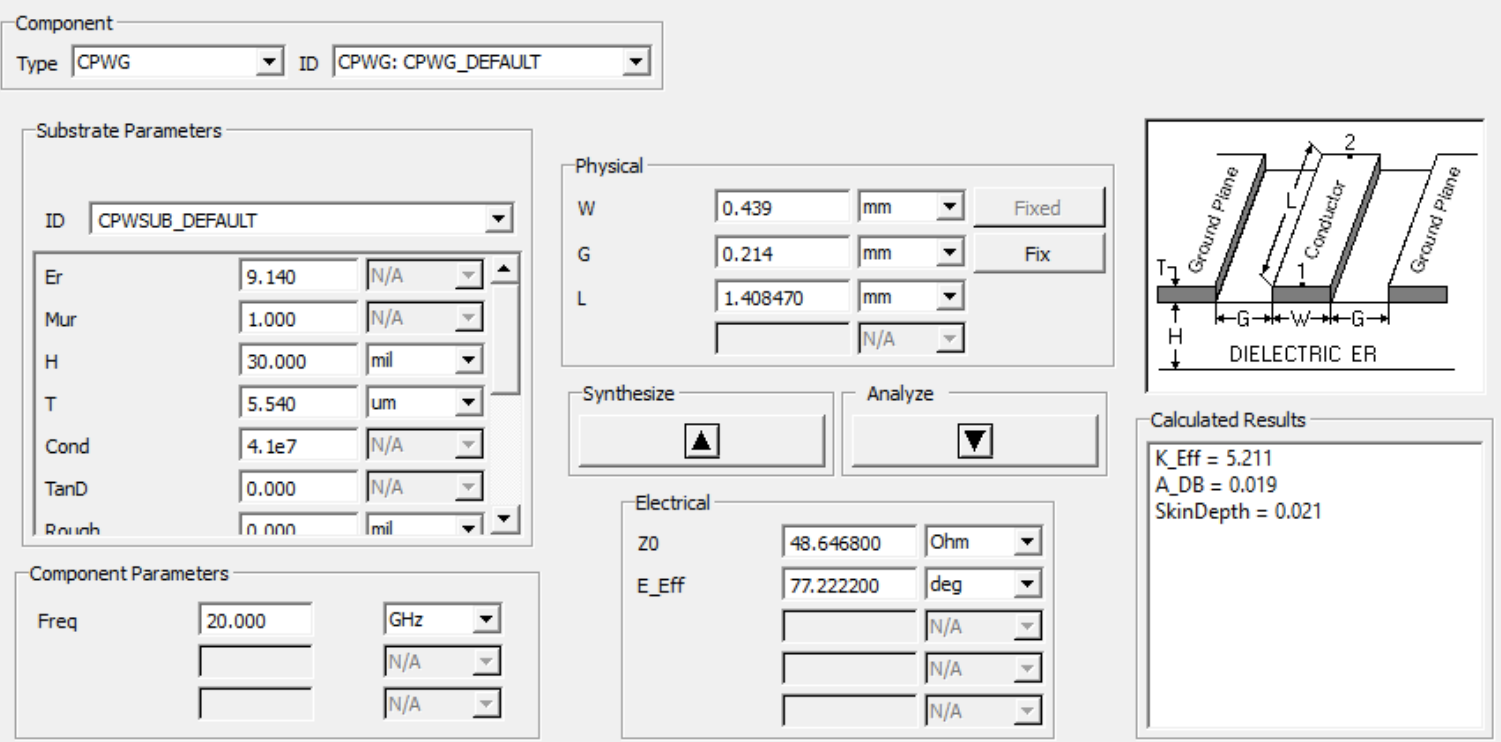

Figure 3-19: Material Parameters for Aluminum Nitride Coplanar Waveguide

K_Eff $=$ Effective Dielectric Constant of Permittivity in AlN Waveguide $=5.211$

$n=$ index of refraction of material $=\sqrt{\epsilon_{r} \mu_{r}}$

$$
\begin{gathered}
\epsilon_{r}=\text { relative permeability of material } \\
\mu_{r}=\text { relative permeability of material } \\
\mu_{r} \approx 1 \text { at optical frequencies [8], therefore } n \approx \sqrt{\epsilon_{r}}
\end{gathered}
$$




$$
\begin{aligned}
& \qquad n=\sqrt{\epsilon_{r}}=\sqrt{5.211}=2.283 \\
& \qquad v_{\text {phase }}=\frac{c}{n}=\frac{c}{2.283}=0.438 \mathrm{c} \\
& \lambda=\text { wavelength of EM wave in Aluminum Nitride substrate = } \\
& \qquad \frac{v_{\text {phase }}}{f}=\frac{0.438 \times\left(\frac{3 \times 10^{8} \mathrm{~m}}{\mathrm{~s}}\right)}{20 \times 10^{9} \mathrm{GHz}}=6.57 \mathrm{~mm} \\
& \text { Length of transmission line }=77.4 \text { degrees }=\left(\frac{77.4}{360}\right) \times \lambda=\mathbf{1 . 4 0 8} \mathbf{m m} \\
& \text { Calculating the length of the coplanar waveguide open stub was a similar } \\
& \text { procedure. The LineCalc tool was used to calculate the dimensions of the } \\
& \text { transmission line to be used. The waveguide would have to be } 0.98 \text { mm in length. } \\
& \text { This turned out to be an impractical design due to non-idealities in the dimensions } \\
& \text { of the waveguide. It was too close to the existing coplanar waveguide cross- } \\
& \text { section dimensions. There likely may not be much of an improvement with it. }
\end{aligned}
$$

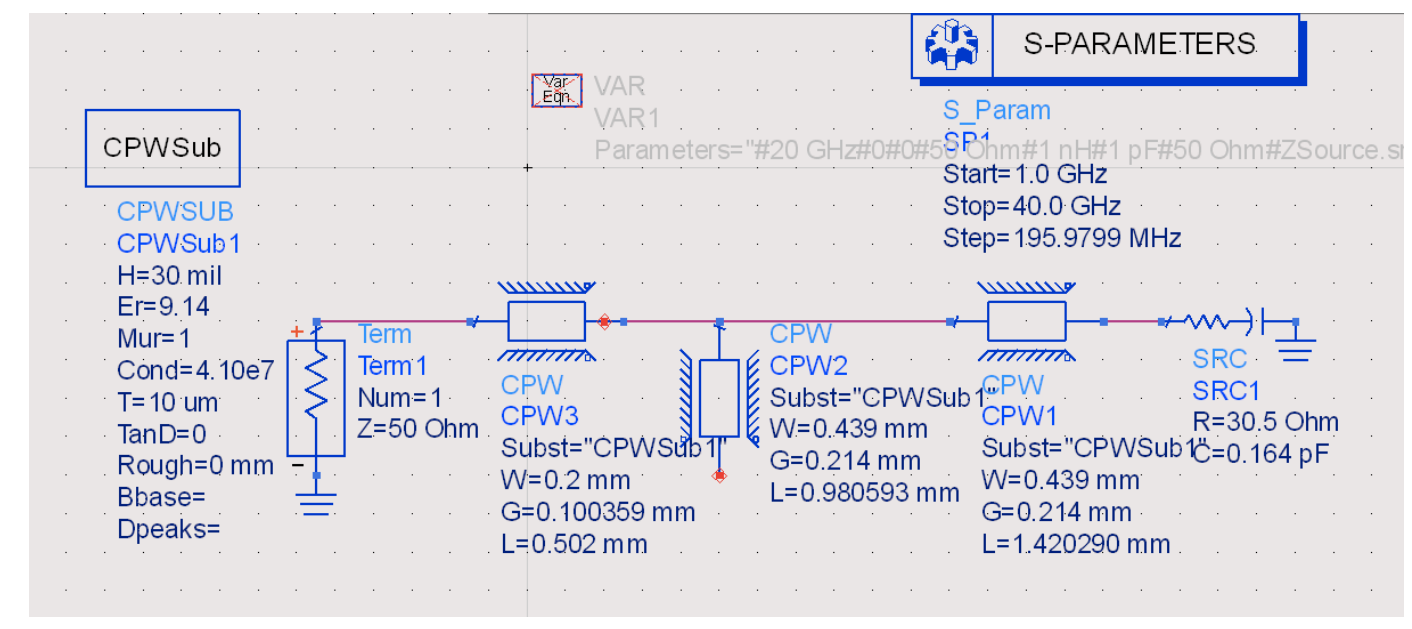

Figure 3-20: Device Model of Single Open Stub Matching Network using a Coplanar Waveguide 
Therefore, a lumped element version of the matching network was considered. After the transmission line directly connected to the load, a lumped element capacitor would be needed with an inductive susceptance of 1.36628 siemens. And so, a $0.2168 \mathrm{pF}$ capacitor would be used.

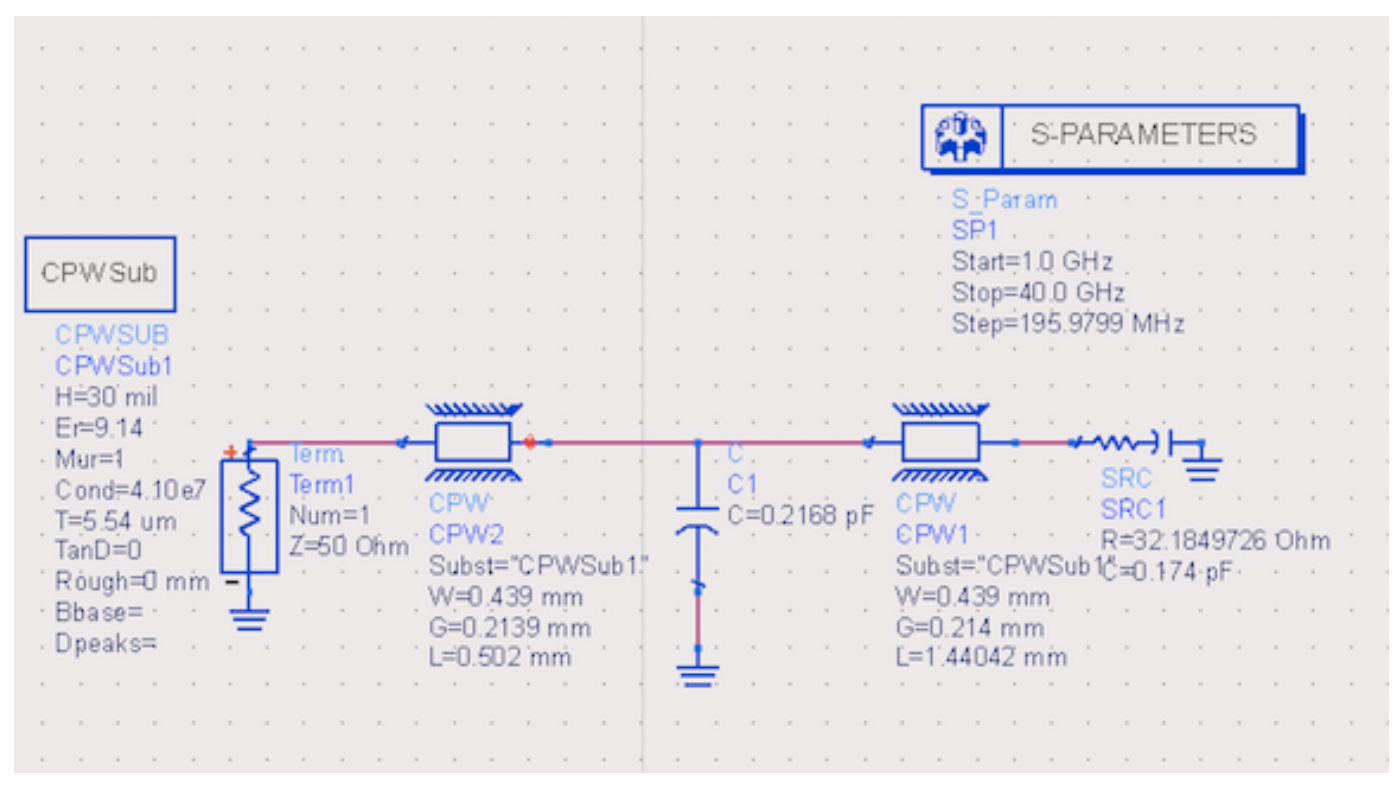

Figure 3-21: Device Model of Single Open Stub Matching Network using Lumped Elements 
$\mathrm{m} 1$

freq $=19.81 \mathrm{GHz}$

$S(1,1)=0.071 /-151.151$

impedance $=Z 0 *(0.881-\mathrm{j} 0.061)$

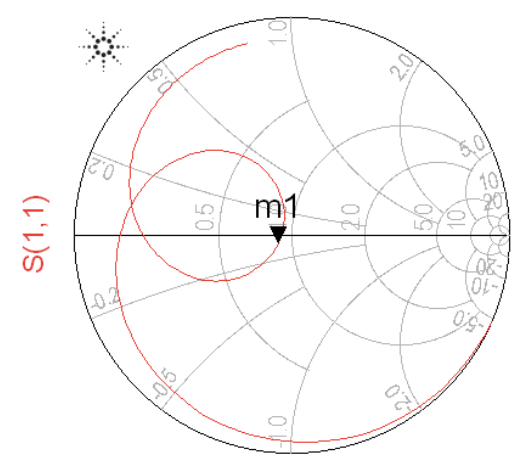

freq $(1.000 \mathrm{GHz}$ to $40.00 \mathrm{GHz})$

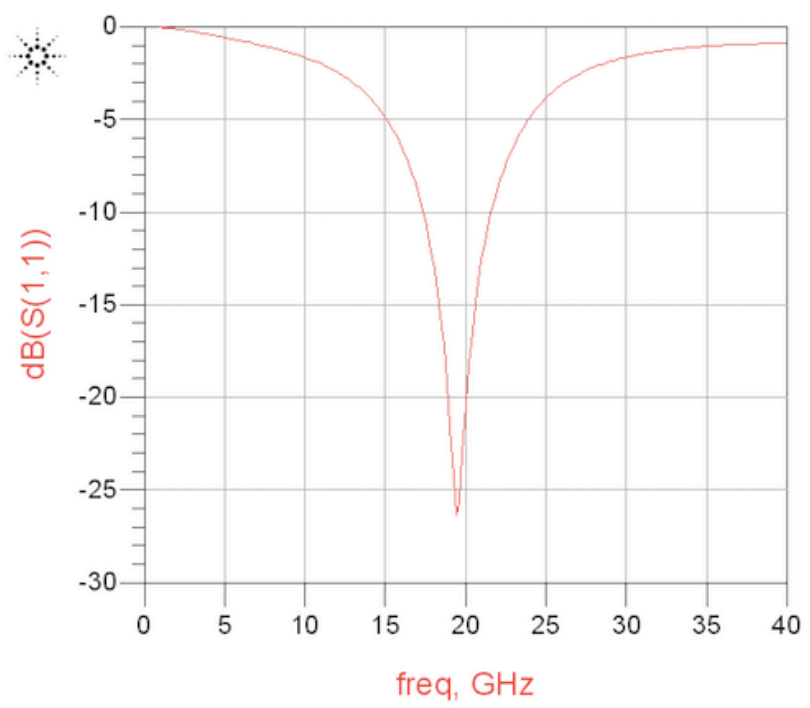

Figure 3-22: Smith Chart and S11 Simulations of Single Open Stub Matching Network using Lumped Elements

In an ideal situation, this would be the capacitor to include in the matching network. But, because the capacitors would need to be wire bonded to the waveguide, the inductance coming from the wire bonds would need to be 
considered. Gold would be used for the wire bonding of the capacitors. Below is the formula for bond wire inductance. [10]

$$
\mathrm{L}=5.08 \times 10-3 * \mathrm{~L} *(\ln (4 * \mathrm{~L} / \mathrm{D})-1)(\mathrm{nH})
$$

For a $1 \mathrm{~mm}$ length and 1 mil diameter wire bond, there would be about $0.81 \mathrm{nH}$ of inductance. Therefore, for about every 1 millimeter, there would be about $0.8 \mathrm{nH}$ of wire bond inductance. Assuming the wire bond that would be used is about $0.5 \mathrm{~mm}$ in length, the inductance across the wire bond would be about $0.4 \mathrm{nH}$. This then was used to calculate an adjusted capacitance of $0.2 \mathrm{pF}$. Also, in order to keep equal coupling in both directions of current flow through the waveguide, two capacitors would be inserted on both sides of the waveguide rather than just on one. These capacitors would simply add in parallel, meaning they would both have half the value of the total capacitance. Two capacitors then were used in the matching network designs that follow, each being $0.1 \mathrm{pF}$, totaling to a equivalent capacitance of $0.2 \mathrm{pF}$.

Interconnect inductance can cause impedance mismatching and reduced bandwidth. [9] This was the case for the first physical realization of the matching network, where the wire bonds added a significant amount of inductance, which created an impedance mismatch. 
In Figure 3-23, a device model of the impedance matching circuit, and Figure 3-24 displays a photo of the first matching network implementation, which included the two $0.1 \mathrm{pF}$ capacitors wire bonded to the center waveguide.

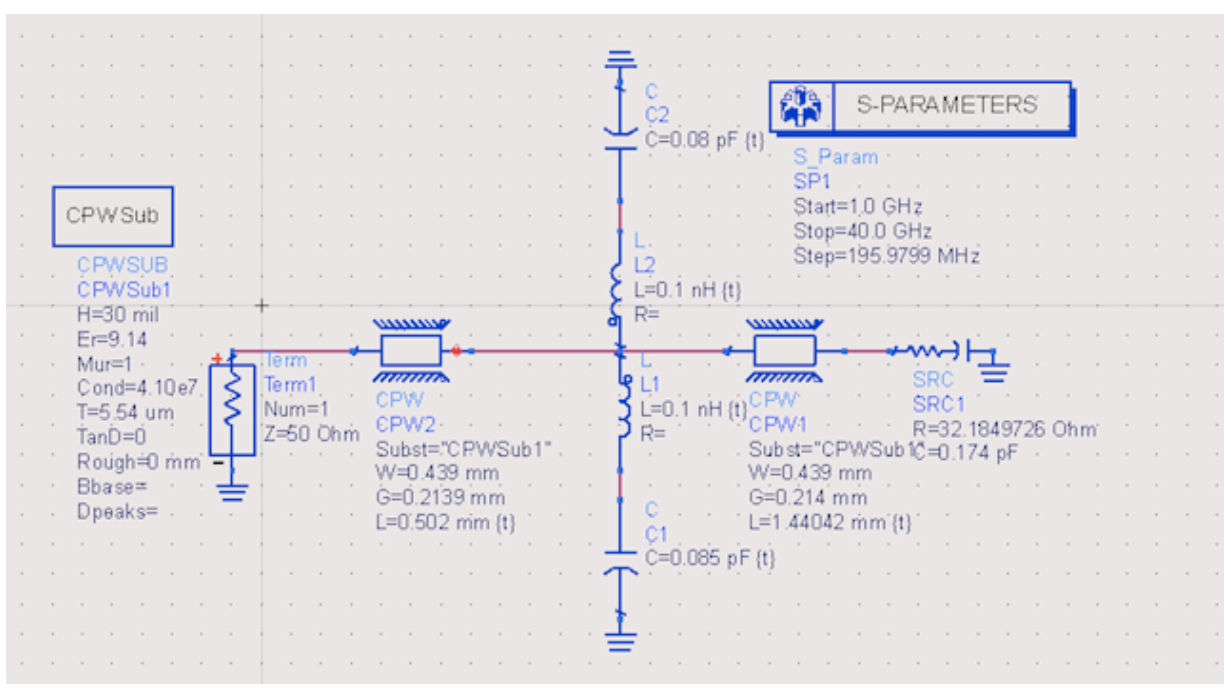

Figure 3-23: Device Model of Matching Network with Wire Bonds

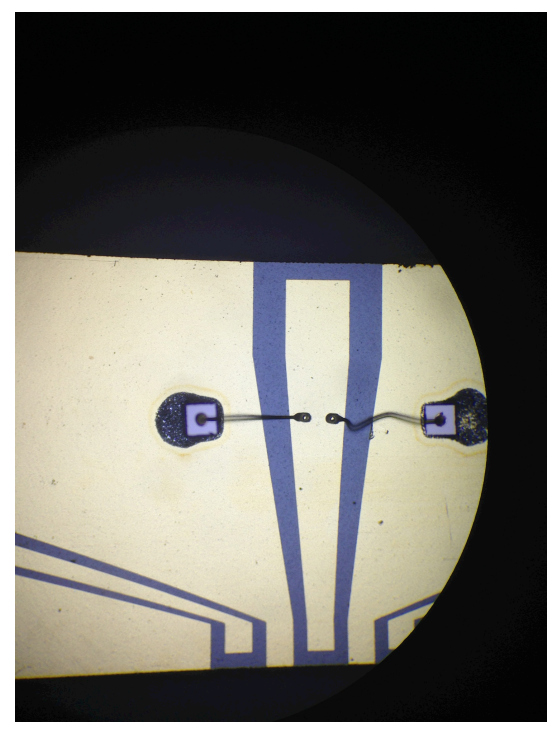

Figure 3-24: Photograph of Matching Network with Wire Bonded Capacitors 
Scattering parameter measurements were taken for the impedance matching network with wire bonds being used to attach the capacitors to the coplanar waveguide. Note that the measurements were made on the coplanar waveguide before it was packaged into the larger copper block module. The test setup included an Agilent N4373C67 GHz Lightwave Component Analyzer, and a pair of $1.5 \mathrm{~mm}$ wide pitch $\mathrm{RF}$ probes that were applied directly onto the waveguide in order to take measurements.

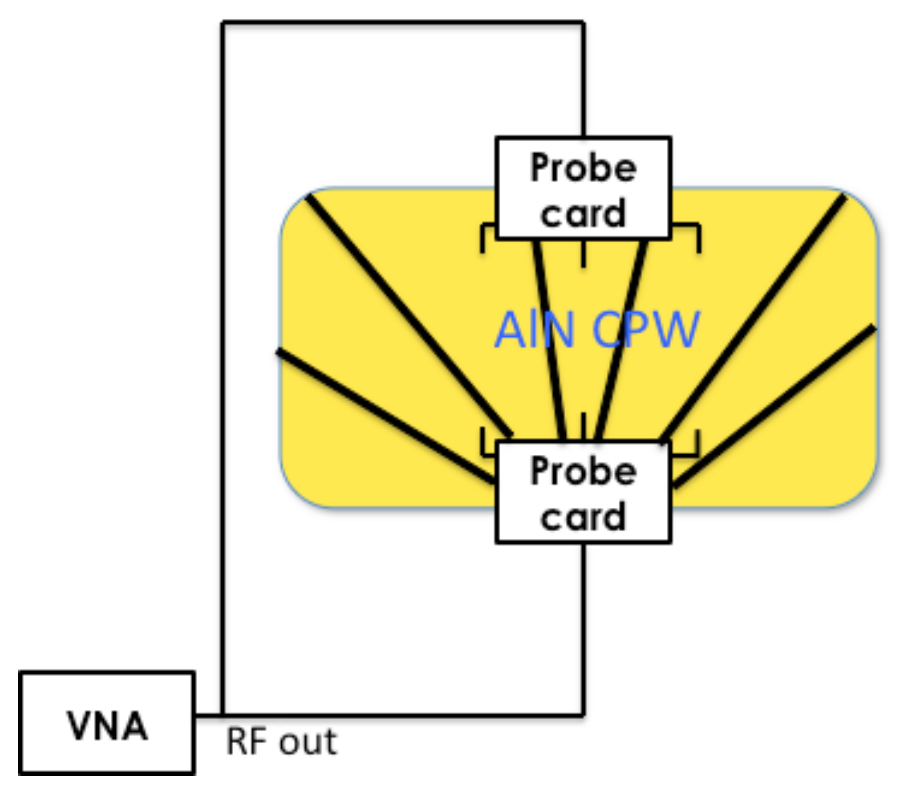

Figure 3-25: Schematic of Test Setup for Matching Network Verification 


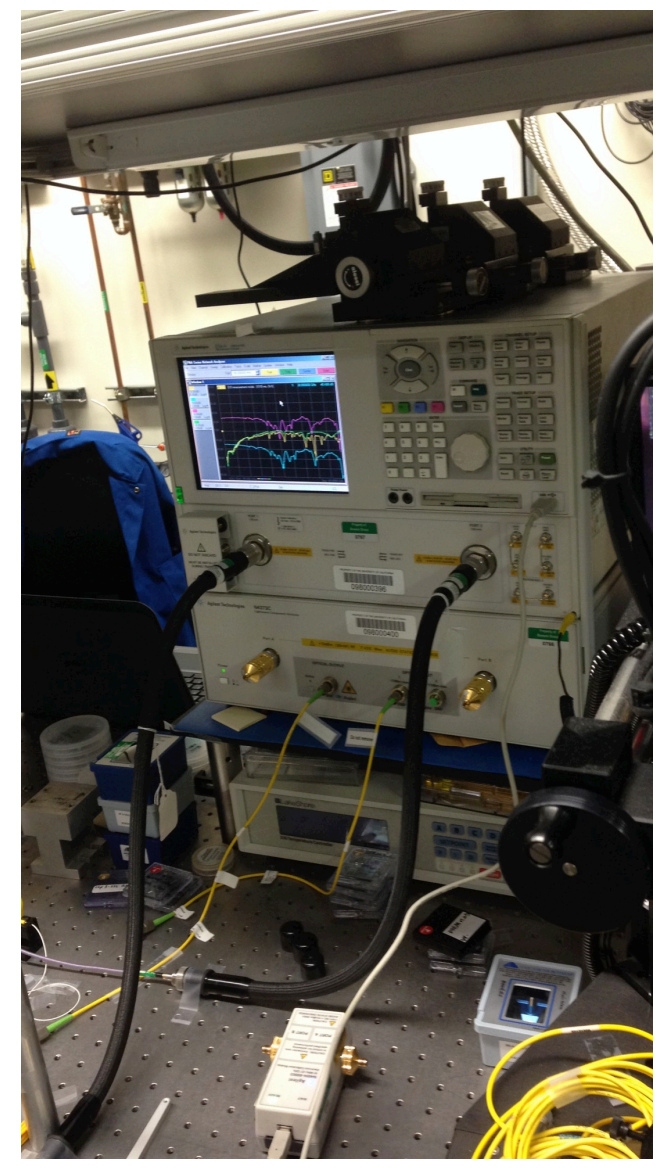

Figure 3-26: Agilent N4373C $67 \mathrm{GHz}$ Lightwave Component Analyzer used to take RF measurements of the waveguide with matching network attached 


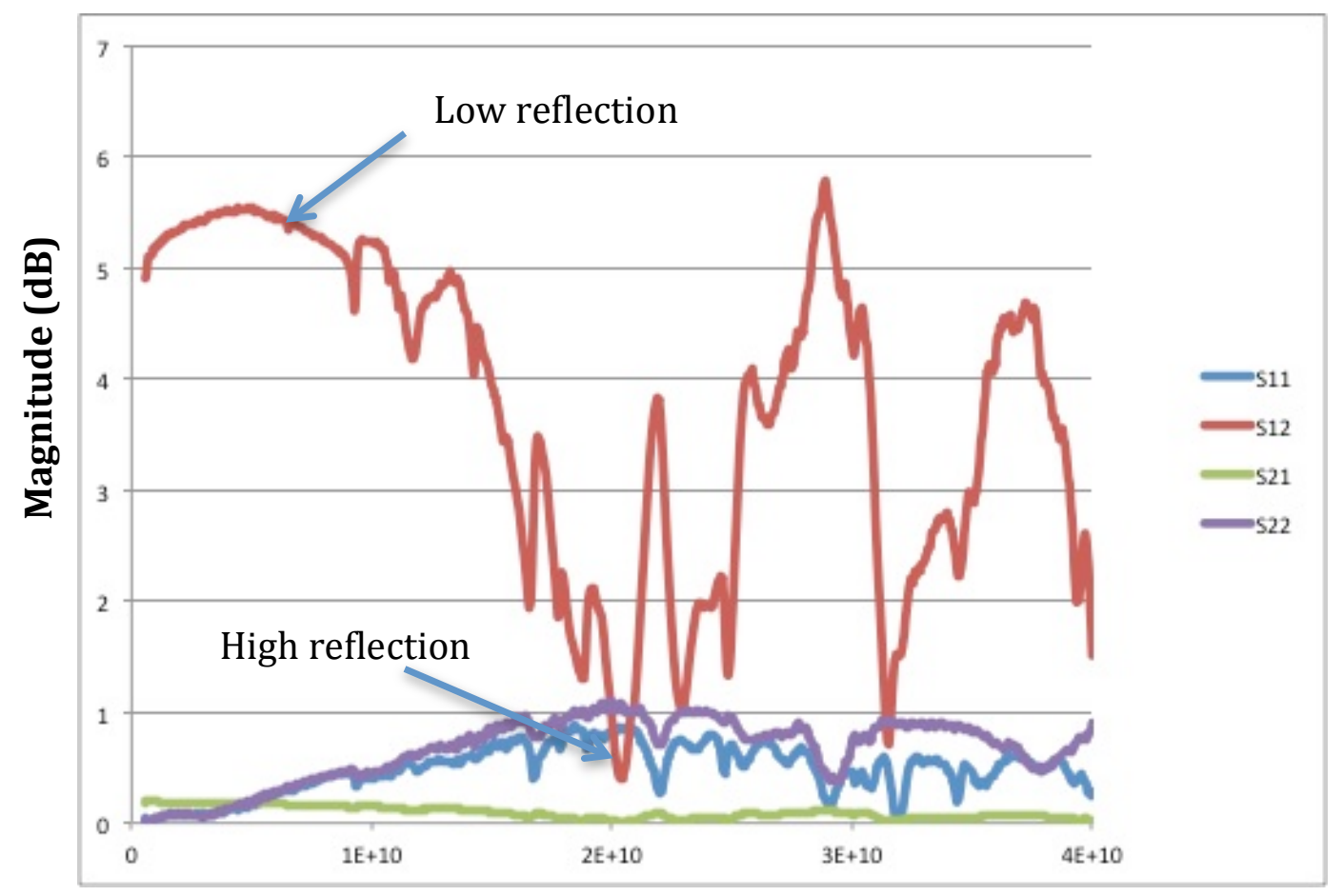

Frequency (Hz)

Figure 3-27: Scattering Parameter Measurements of waveguide with ribbon bonded capacitors attached. Calibration adds a positive offset in the magnitude scale, and therefore, the magnitudes measured are not absolute.

The measurements reveal information regarding the matching network, such as the inductances of the wire bonds being used to connect the capacitors to the center waveguide. The two sequential dips in the S12 measurements shown in Figure 3-27 are the LC resonant frequencies of the two capacitive branches of the impedance matching network. At these frequencies, the capacitor and the inductor in each branch become equal in magnitude and, in result, cancel each other out. The branches become shorts, and therefore, reflect the whole RF signal back to the generator. That is why a high transmission loss is observed at those frequencies. 


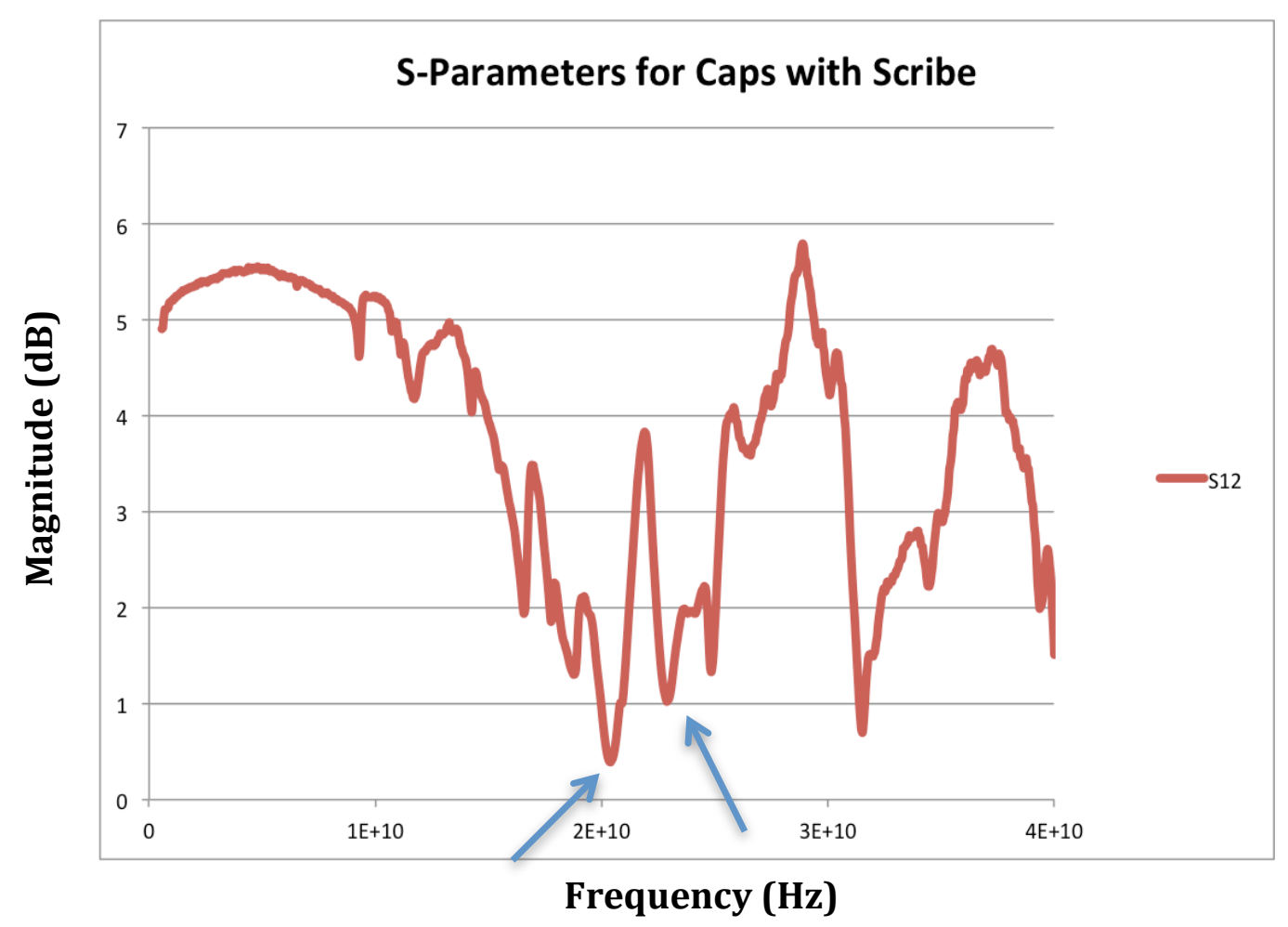

Figure 3-28: Scattering Parameter Measurements of waveguide with ribbon bonded capacitors attached. Blue arrows refer to the resonant frequencies of the LC branches of the matching circuit.

Knowing that the capacitors are both $0.1 \mathrm{pF} \pm 10 \%$, the inductances were calculated using ADS. Simulations were made of the circuit, and the inductance of each wire bond was adjusted until a similar S12 was simulated in comparison the actual S12 measurements. 


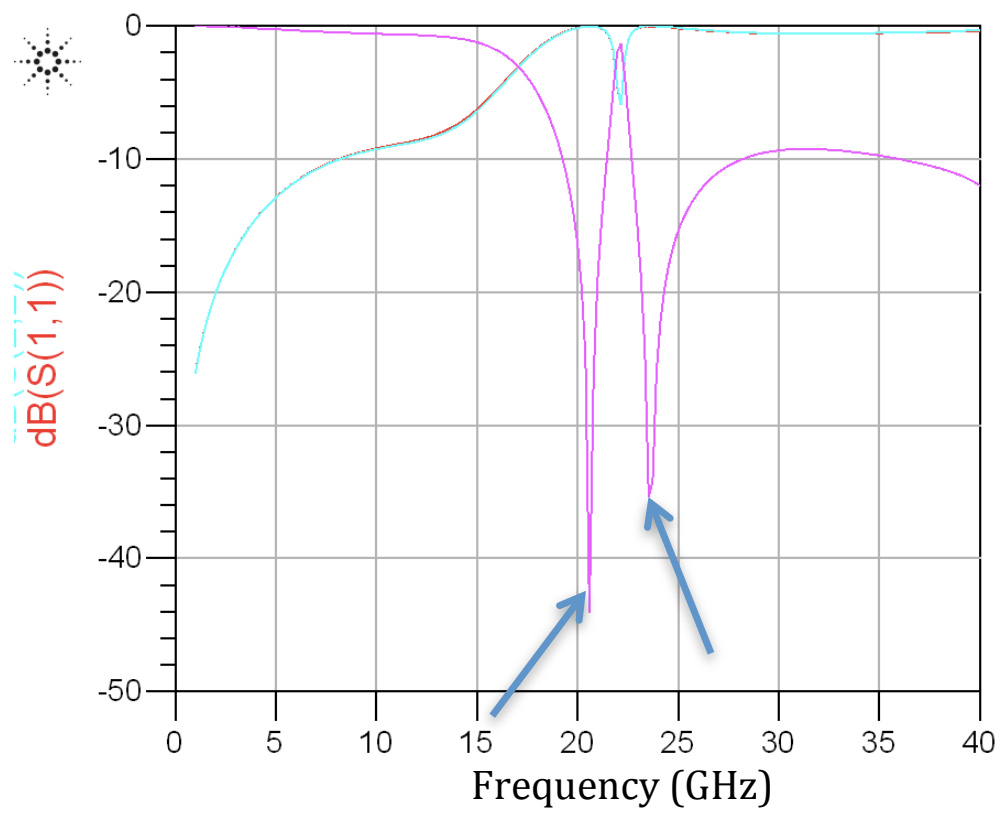

Figure 3-29: Simulated S11 (red) and S12 (blue) Measurements with Blue Arrows Pointing out dips at Resonant Frequencies of LC branches of matching Circuit

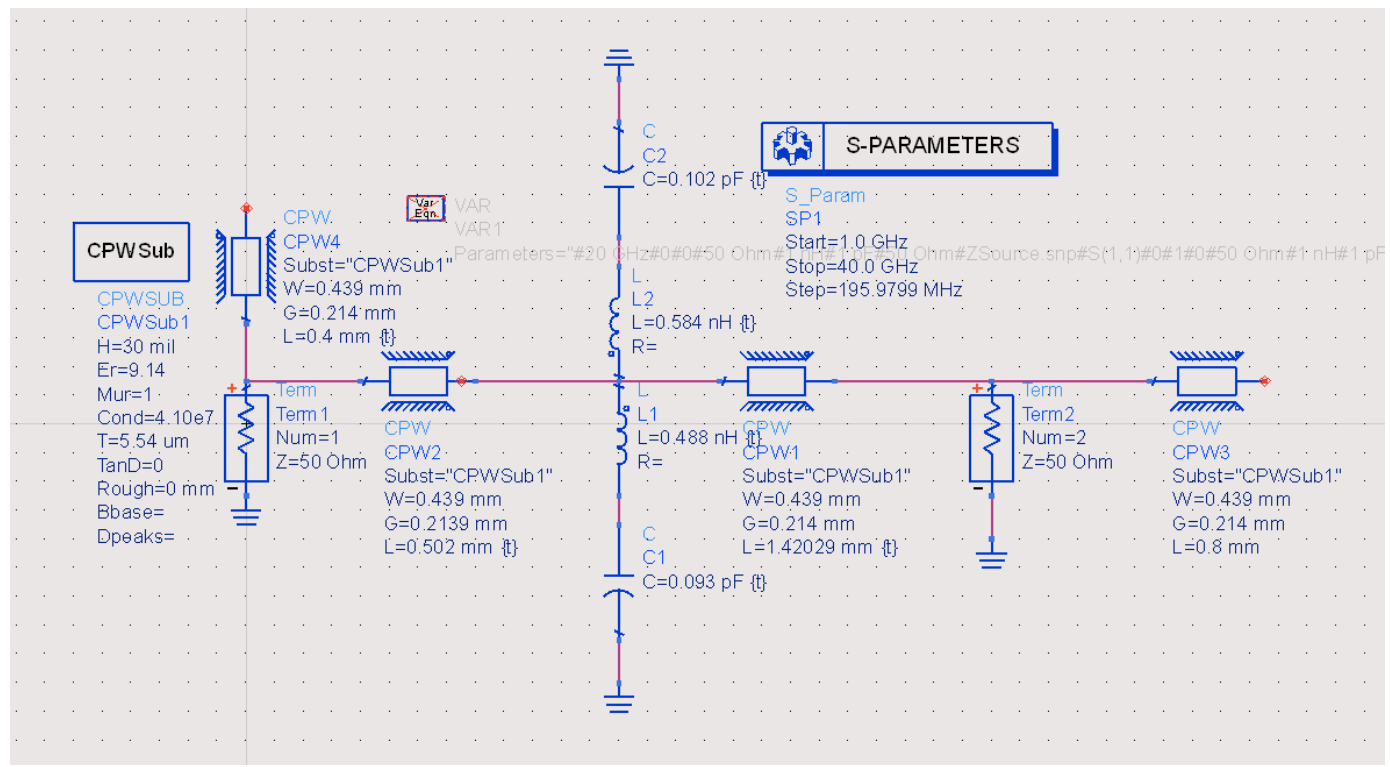

Figure 3-30: Device Model of Test Setup in order to Simulate Test Results

The inductances of the wire bonds were found to be $0.584 \mathrm{nH}$ and 0.488

$\mathrm{nH}$. When these inductances were used to simulate the S11 of the matching 
network, the match was shown to be far less of a match than without the wire bonds, as displayed in Figure 3-30.
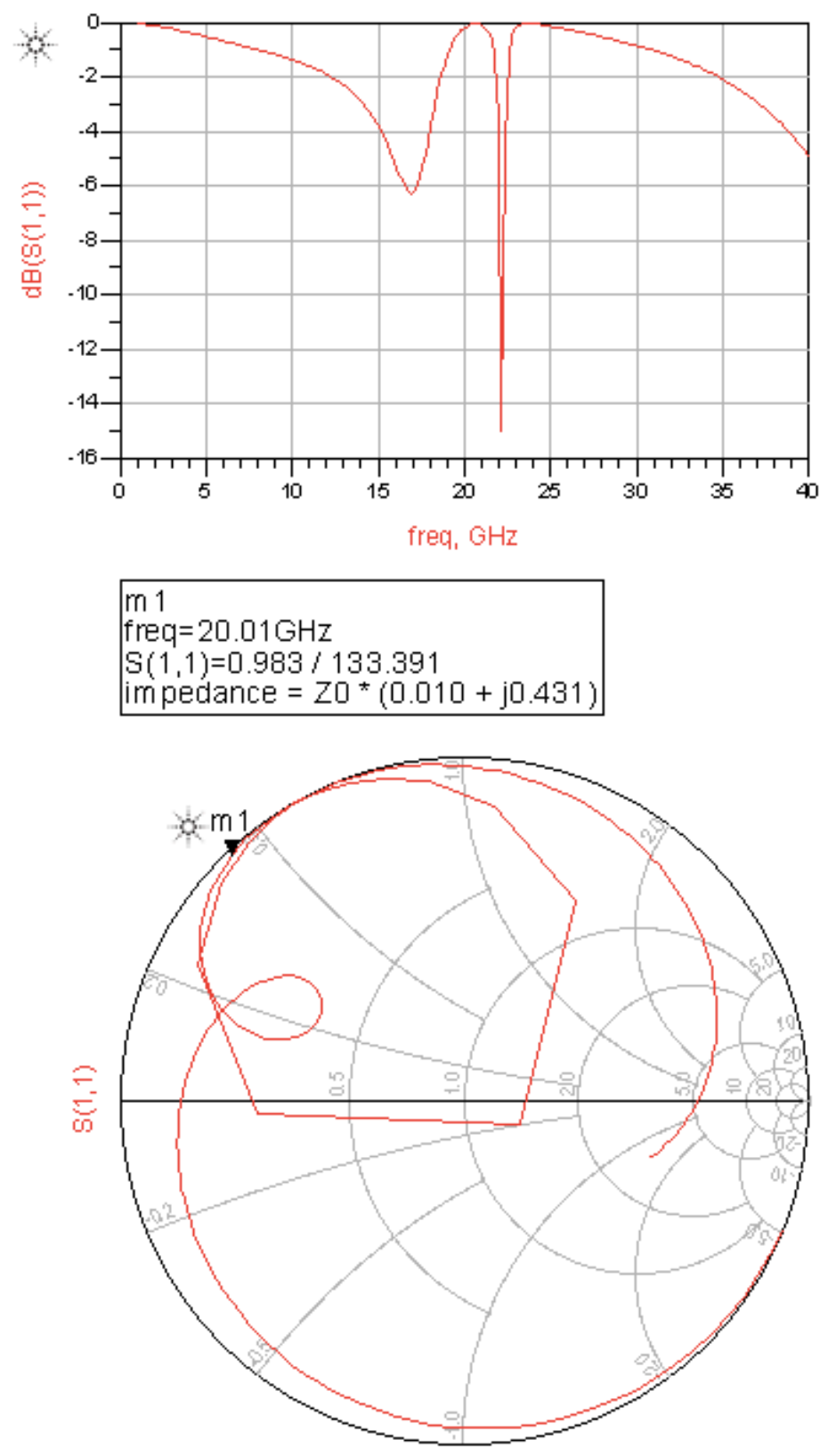

Figure 3-31: S11 simulation measurements for wire-bonded matching network. Marker $\mathrm{m} 1$ shows the load impedance at $20 \mathrm{GHz}$, which is far from a $50 \Omega$ match. 
Therefore, the issue of wire bond inductance changing optimal matching conditions needed to be solved. So, instead of wire bonds, ribbon bonds were used in the next matching network application. Ribbon bonds offer inductance two or three less times that of a wire bond of equal length [9]. They may even carry a small amount of capacitance, which actually would be advantageous in this scenario, due to a need for increased capacitance as well as a reduction in interconnect inductance.

So, capacitors were then ribbon bonded to the center waveguide. The ribbon bonds were also made as short as possible to minimize inductance. In Figure 3-32, a photo of the new matching network is shown.

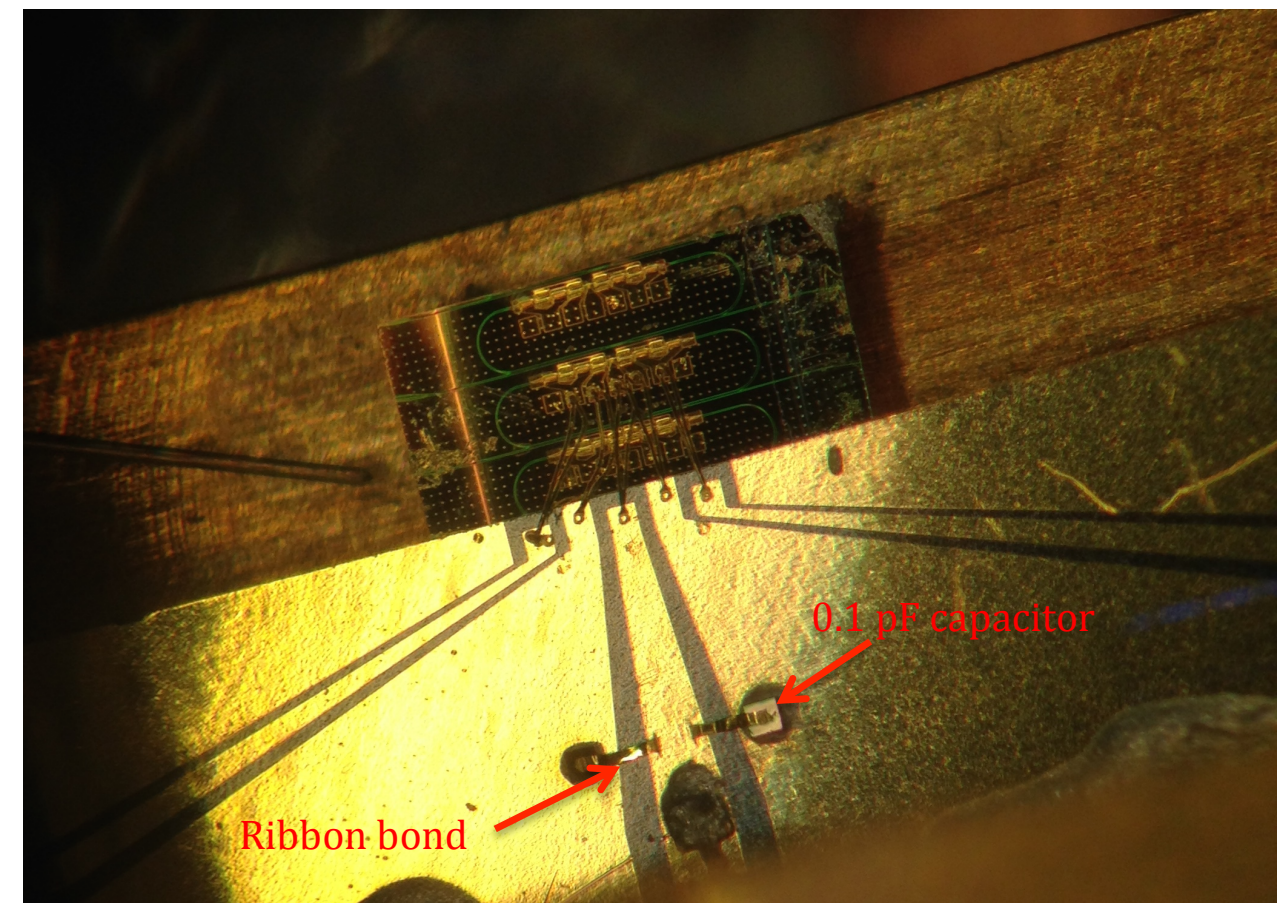

Figure 3-32: Photo of matching capacitors ribbon bonded to coplanar waveguide 


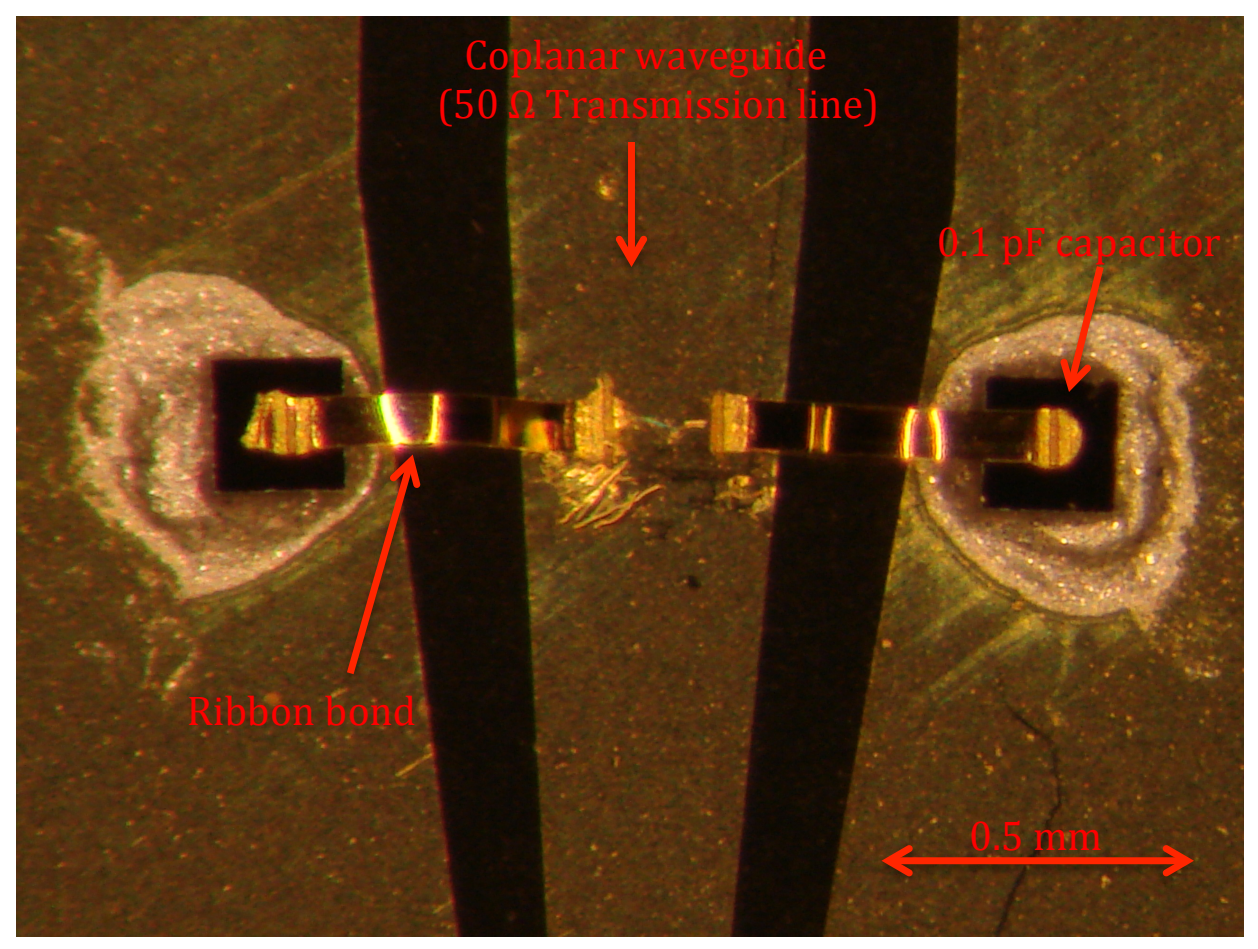

Figure 3-33: Close-up of Gold Ribbon Bonds

S-Parameter measurements were taken once again, and in a similar way to the wire bonds, the inductances of the ribbon bonds were calculated and found to be $0.13 \mathrm{nH}$ and $0.21 \mathrm{nH}$. This was a significant improvement from the wire bonds, and simulations showed that indeed a much better match was made with the ribbon bonds. This time the coplanar waveguide was package in the copper block module with the laser and the RF and DC pins bonded and attached. The circuit model is shown in Figure 3-34. 


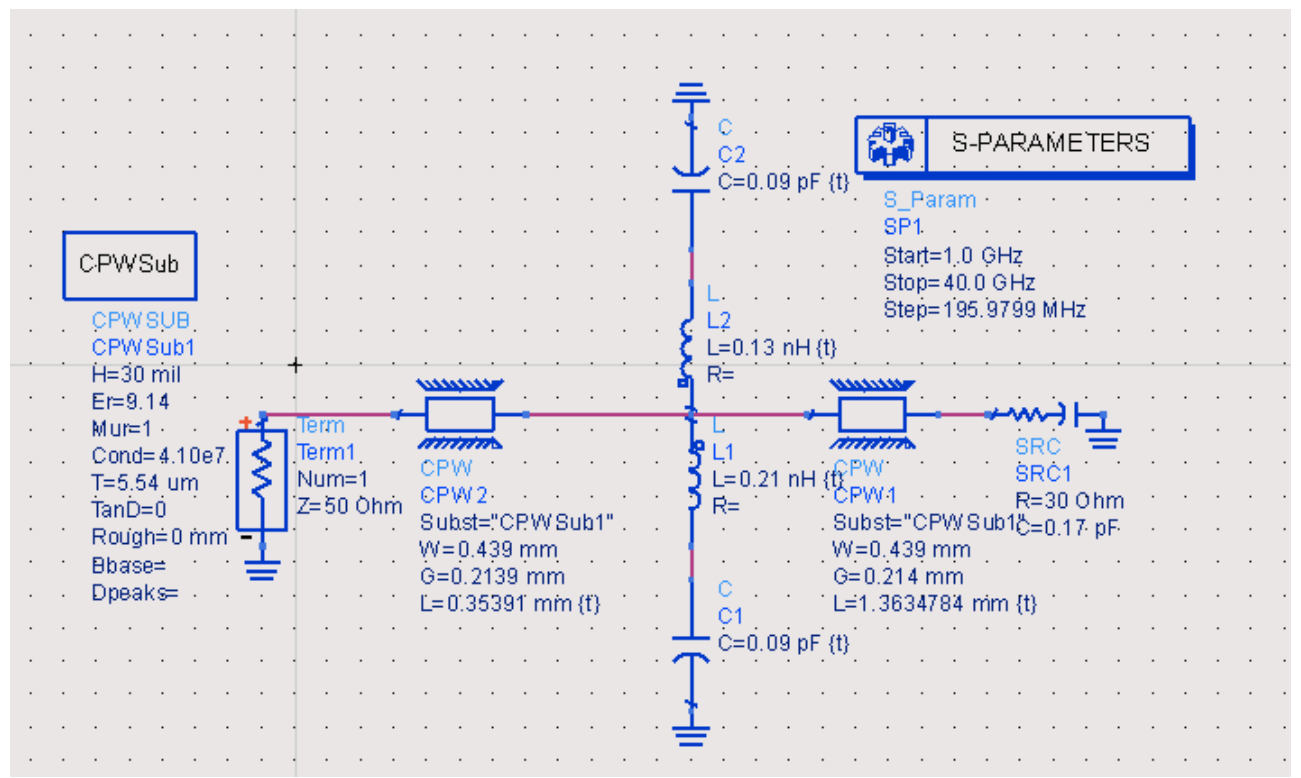

Figure 3-34: Device Model of matching network with ribbon bonded capacitors 
$\mathrm{m} 2$

freq $=20.01 \mathrm{GHz}$

$S(1,1)=0.093 /-162.789$

impedance $=Z 0^{*}(0.835-j 0.047)$
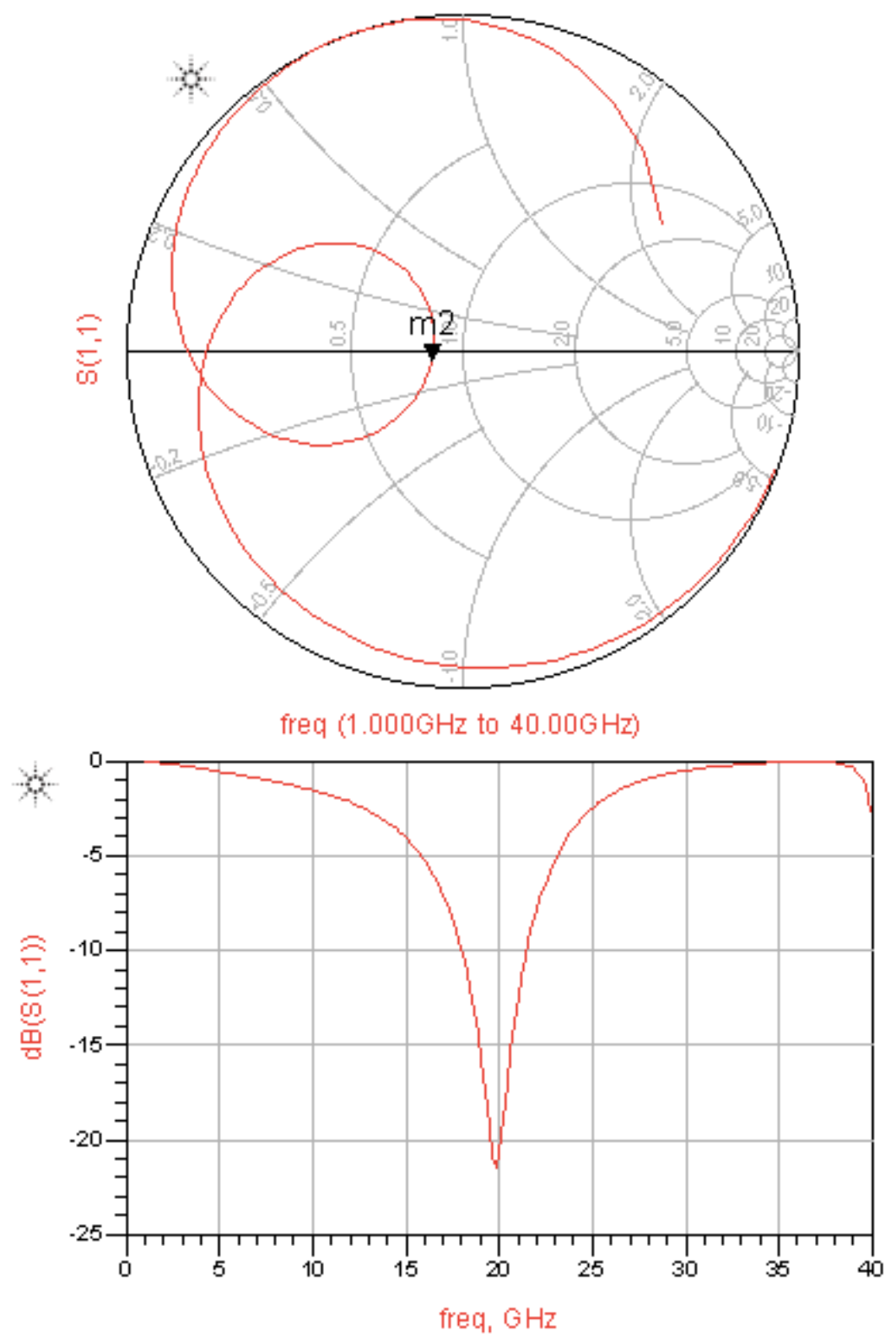

Figure 3-35: S11 simulation measurements for matching network with ribbon bonded capacitors 
With this matching network, the simulations show there would be a return loss of $22 \mathrm{~dB}$. This means that less RF power is reflected, and therefore, less power is required to achieve certain performance from the device. Without the matching network, about $34.9 \%$ of the RF signal is reflected from the load, and therefore, only, $65.1 \%$ is transmitted. The impedance matching network would theoretically have all of the RF power delivered to the load. In this case, there is about $7.6 \mathrm{~dB}$ return loss without the matching network, and an estimated $22 \mathrm{~dB}$ return loss with the matching network. Therefore, there should be a $14.4 \mathrm{~dB}$ improvement of RF power delivered to the load. 


\section{Chapter 4: Results and Analysis}

\subsection{Test Setup and Measurements}

The first test setup was put together to begin measuring the laser diode for any improvement in output RD power. The test took S11 measurements of the complete module while the laser diode mode-locks. The laser diode was biased using the RF and DC pins on the copper block module. Two DC power supplies were used as well as the internal frequency generator coming from the Agilent N4373C $67 \mathrm{GHz}$ Lightwave Component Analyzer, which generated a $20 \mathrm{GHz}$ signal. The DC bias signal as well as the RF signal were both put into a bias tee, and then used to bias the saturable absorber section of the diode. Bias conditions for when the diode was mode-locking were noted before the experiment, so that measurements can be taken for when the diode mode-locks. Figure 4-1 shows a schematic diagram of this test setup.

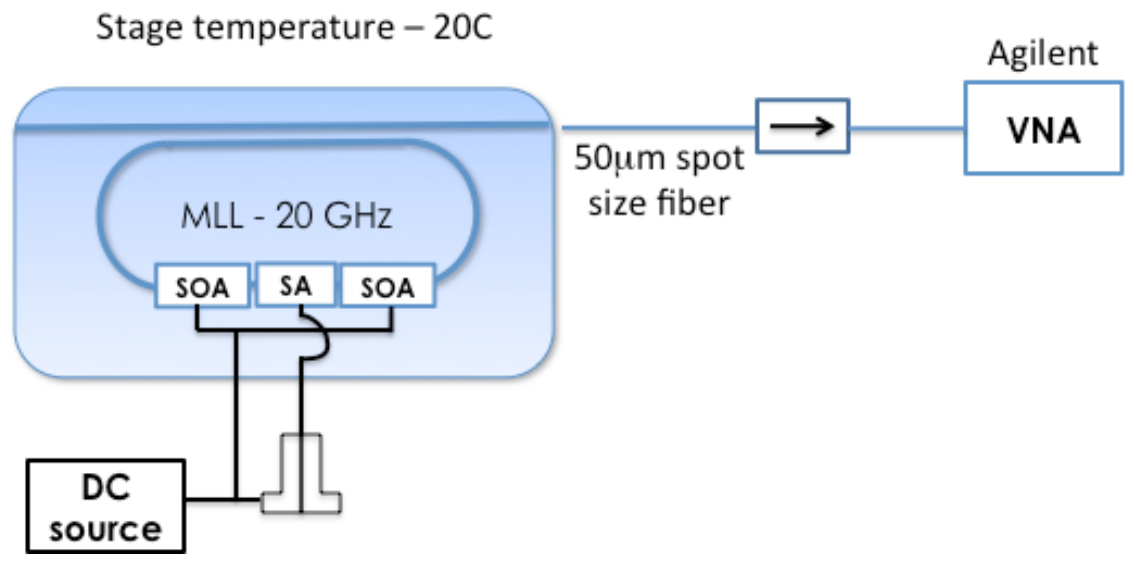

Figure 4-1: Schematic of the test setup used to measure S11 of laser 
In Figure 4-2, are Smith Chart measurements of the S11 coming from the package, showing the impedance of the laser diode in the package including the ribbon bonded matching network. This shows that the impedance is indeed much closer to $50 \Omega$ than it was before, and indicates that the better match should translate to a reduced drive power requirement for active mode-locking to occur in the laser. The reason the match is not perfect is due to the location of the capacitors not being as accurately placed as possible, as well as inductances coming from the ribbon bonds. Also, the capacitors also have a certain tolerance. Plus, the package is and is not a perfectly ideal $50 \Omega$ transmission line due to material defects and mismatches in impedance. This will be explained in more depth in Chapter 5. 


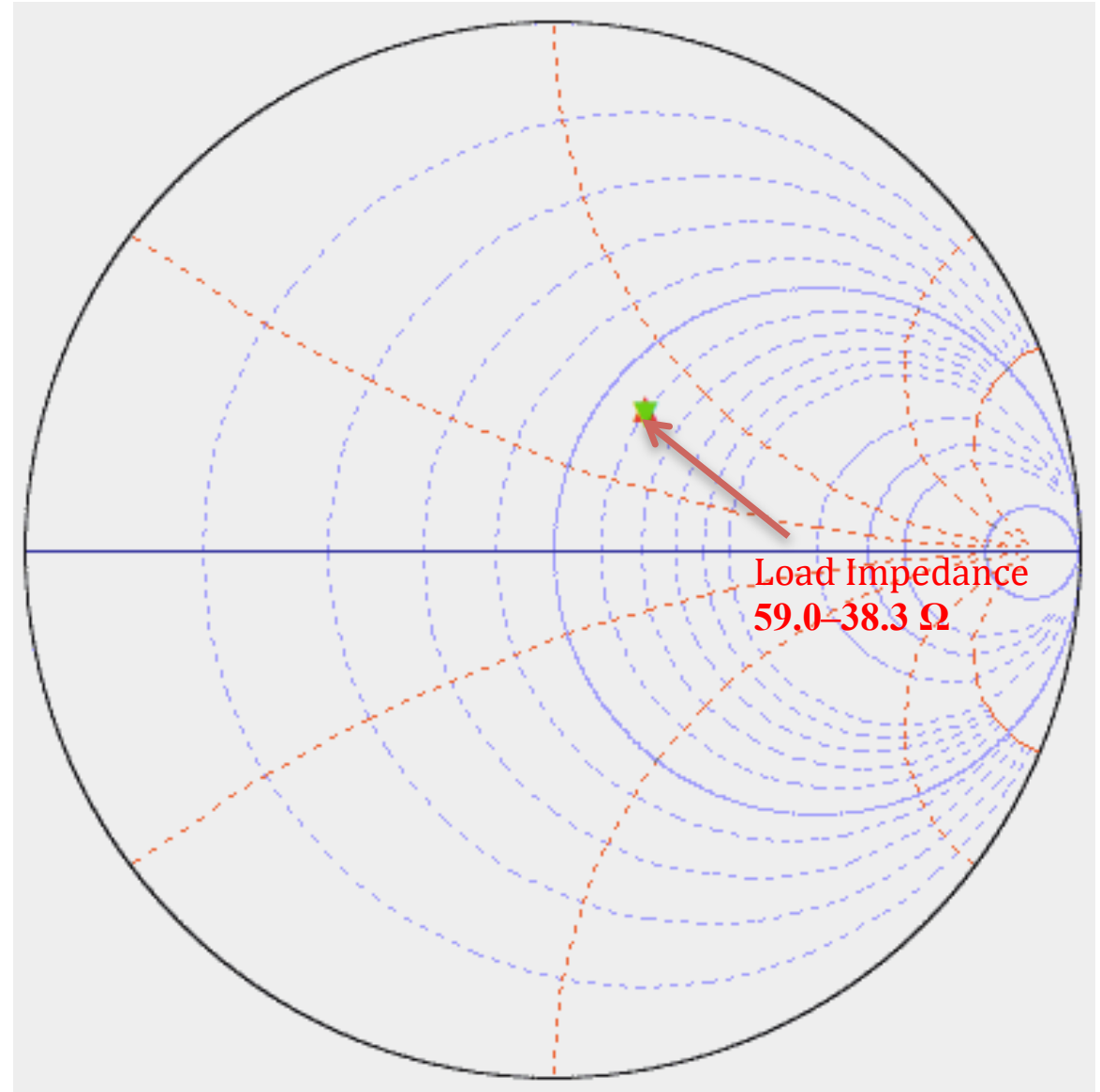

Figure 4-2: The impedance of the diode now is $59+\mathrm{j} 38.3 \Omega$, which is much closer to a $50 \Omega$ match than was the initial load impedance of $30-\mathrm{j} 46.4 \Omega$

Next, the copper block package, which contained the waveguide and the laser diode, was set up on a stage under a 10-60X microscope. The laser diode was then biased using the RF and DC pins on the copper block module. Two DC power supplies were used as well as a Rhode \& Schwartz Signal Generator that generated a $20 \mathrm{GHz}$ signal. The DC bias signal as well as the RF signal were both put into a bias tee, and then used to bias the saturable absorber section of the diode. A $2 \mu \mathrm{m}$ lensed fiber was placed on a metal fiber boat and set up on the 
stage in order to collect light coming from the laser cavity's facet. Then, an Newport optical power meter read the optical power coming from the fiber. This was used to maximize coupling, as the fiber's position was adjusted until a maximum power reading was measured on the optical power meter.

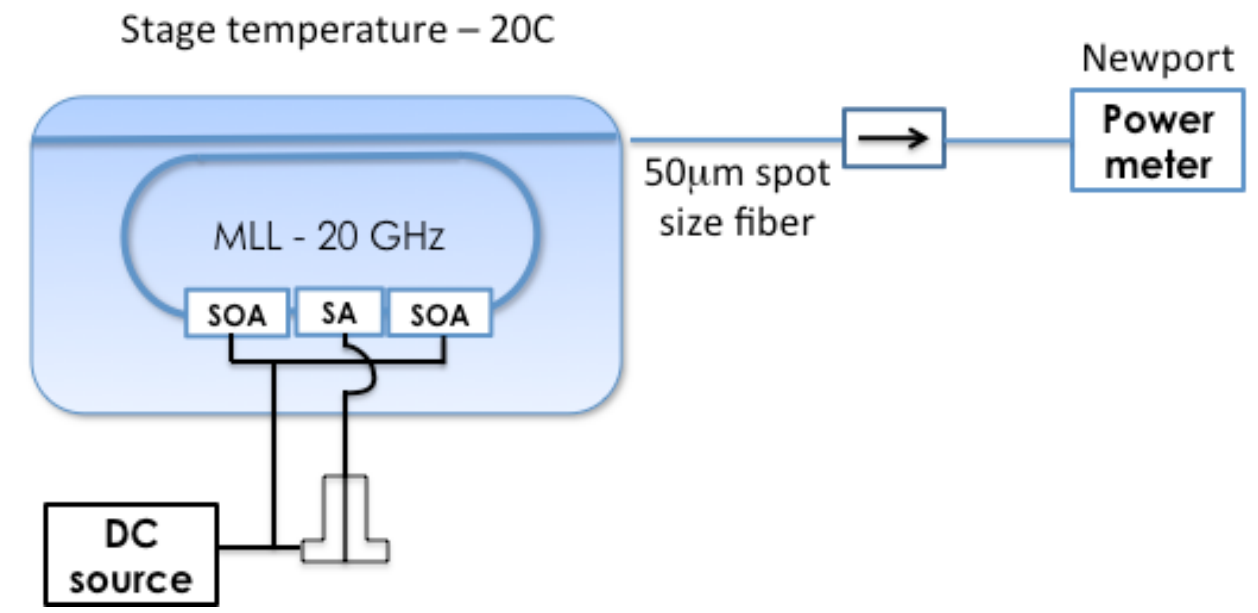

Figure 4-3: Schematic of the test setup used to maximize coupling to lensed fiber

The lensed fiber is then followed by an Erbium Doped Fiber Amplifier (EDFA) an optical filter and a high-speed photodetector, in that order. The EDFA was used to boost the output optical signal coming from the optical fiber. The optical filter is used to match the output wavelength of the laser light in order maximize the gain from the EDFA. The high-speed photodetector is then connected to an Electrical Spectrum Analyzer, which is used to measure the laser's RF power. The packaged diode module was set on top of a TEC on a copper stage, which also had a thermistor taped to the module in order to keep 
track of its temperature. The TEC then kept the temperature of the diode steady at $19.8^{\circ} \mathrm{C}$. The saturable absorber was biased at $-0.9 \mathrm{~V}$ and the SOAs were biased at $191 \mathrm{~m}$, while the RF source signal, used to actively mode-lock the laser, was producing $8 \mathrm{dBm}$ of RF power at around $20 \mathrm{GHz}$. First, measurements were made on the case on which the ribbons were bonded to the capacitors on the Aluminum Nitride waveguide. Figure 4-4 shows a schematic of this test set up, and Figures 4-5 and 4-6 display the measurements taken.

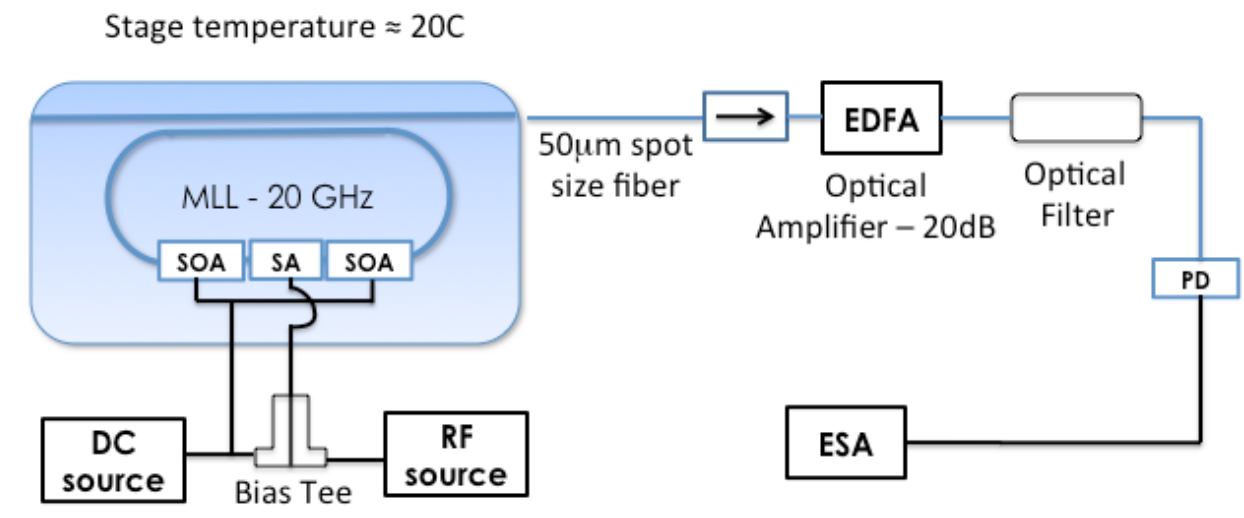

Figure 4-4: Schematic of the final test setup 


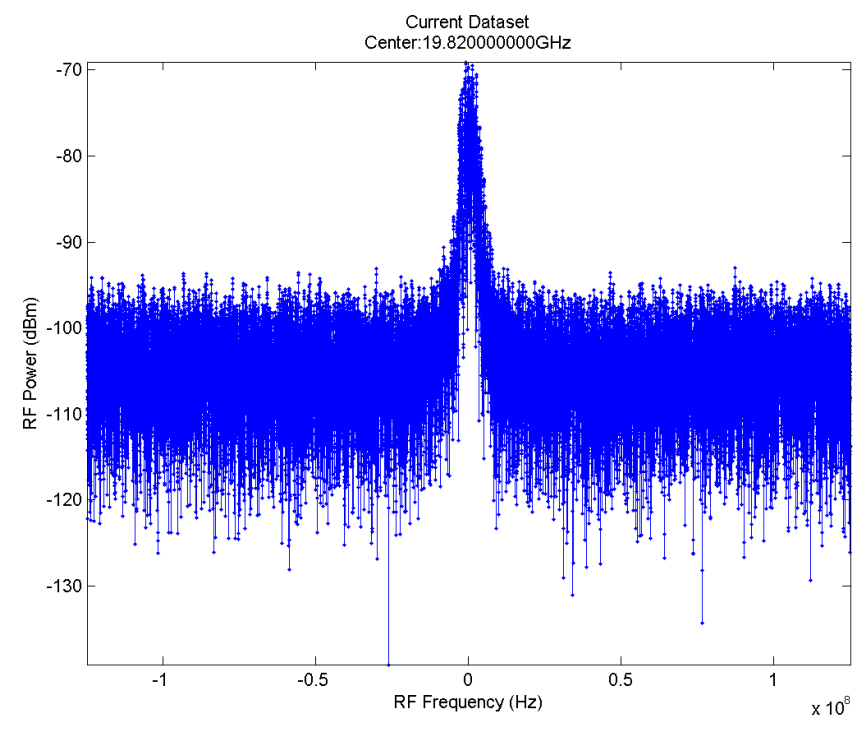

Figure 4-5: Frequency spectrum of the diode with ribbon bonded capacitive matching network attached and passively mode locking. Saturable absorber was biased at $-1 \mathrm{~V}$, and SOAs were biased at $177 \mathrm{~mA}$. Frequency span $=100 \mathrm{MHz}$, resolution bandwidth $=20 \mathrm{kHz}$.

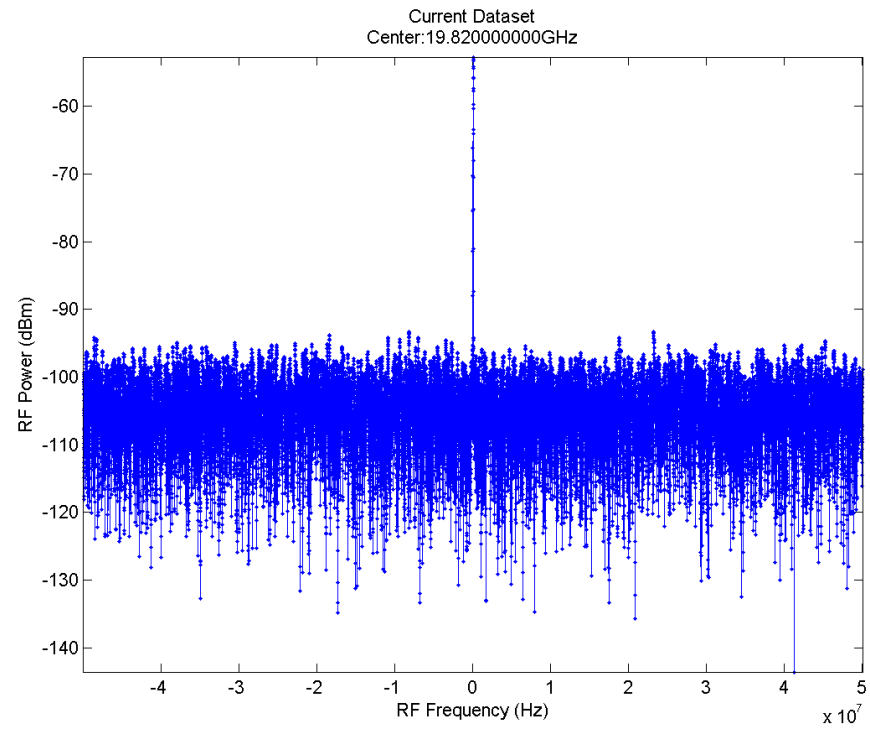

Figure 4-6: Frequency spectrum of the diode with ribbon bonded capacitive matching network attached and actively mode locking. Saturable absorber was biased at $-1 \mathrm{~V}$, and SOAs were biased at $177 \mathrm{~mA}$. RF signal generated at $8 \mathrm{dBm}$. Frequency $\operatorname{span}=500 \mathrm{MHz}$, resolution bandwidth $=20 \mathrm{kHz}$. 
Then, the ribbons were removed using needle nosed pliers, and the same measurements were then taken.

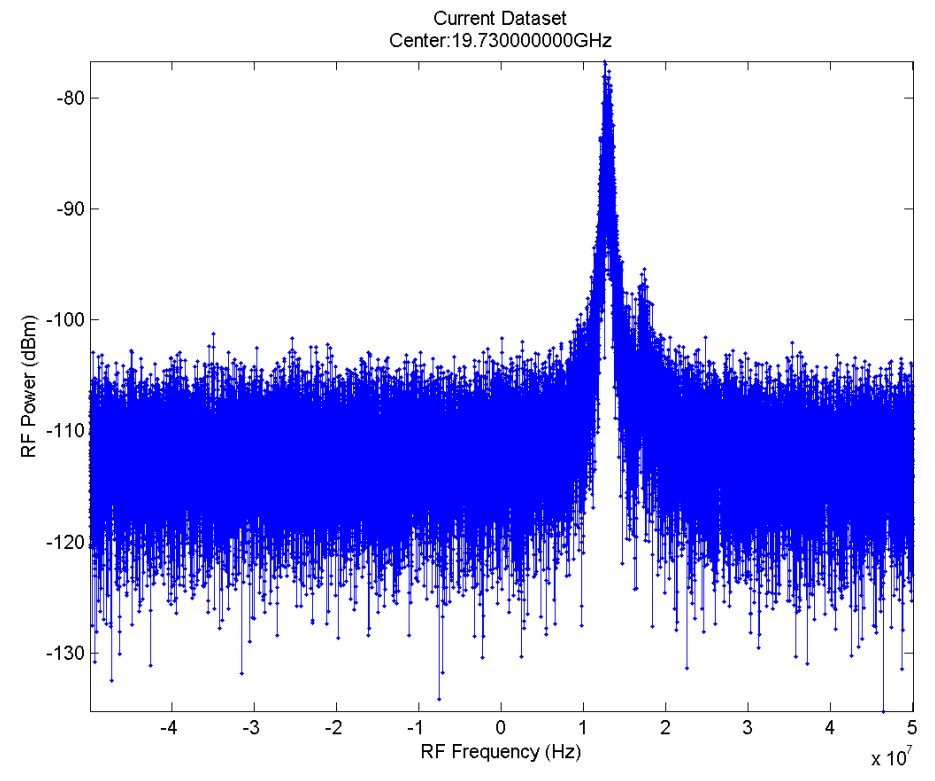

Figure 4-7: Frequency spectrum of the diode with ribbon bonded capacitive matching network removed and passively mode locking. Saturable absorber was biased at $-1 \mathrm{~V}$, and SOAs were biased at $177 \mathrm{~mA}$. Frequency span $=100 \mathrm{MHz}$, resolution bandwidth $=20 \mathrm{kHz}$.

Several of these measurements were taken under different frequency spans and resolution bandwidths when the laser was actively mode-locking. Using those measurements, a phase noise plot was generated for the cases with and without the ribbon bonds. In Figure 4-8, a plot of the phase noise is shown, displaying about a $10 \mathrm{~dB}$ improvement in phase noise. 


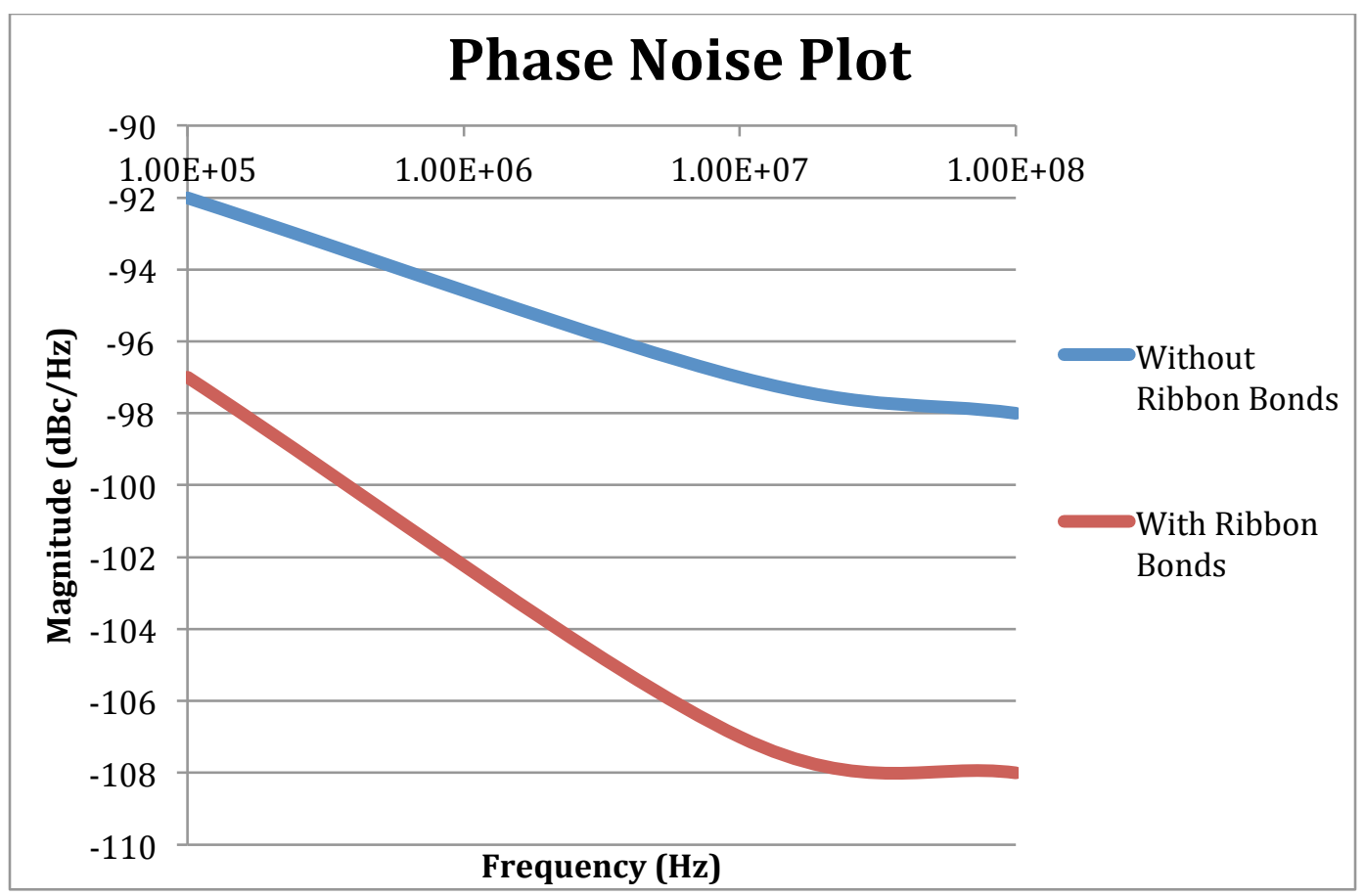

Figure 4-8: Phase noise for the laser with ribbon bonds attached to coplanar waveguide and ribbons bond not attached to the coplanar waveguide in module.

\subsection{Analysis of Results}

A direct comparison was then made between the case with the ribbon bonds attached to the coplanar waveguide and the case with the ribbon bonds removed from the waveguide. A $6 \mathrm{~dB}$ increase in the laser's output RF power measured from a spectrum analyzer was observed at the mode-locking frequency when the impedance matching network was inserted onto the module carrying the device. The optical coupling power was different for both cases. With the wire bonds, the optical coupling was at about $0 \mathrm{~dB}$, and without the wire bonds, the optical coupling was maximized at about $-2 \mathrm{~dB}$. Taking this into account, the 10 
dB difference observed in optical peak powers shown in Figure 4-9 actually differ by $6 \mathrm{~dB}$.

Also, as shown in Figure 4-8, the ribbon bonds also caused for lower phase noise.

The phase noise was improved by about $10 \mathrm{~dB}$ at a frequency offset of a $100 \mathrm{MHz}$.

The close-in phase noise, for the case with the ribbon bonds, has a steeper slope

than without the ribbon bonds, so the phase noise also drops more quickly. In

other words, the phase of the laser is more stable and stays closer to the mode-

locking operating frequency over time. This also means that timing jitter is also

reduced. As, phase noise is a frequency-domain measurement, timing jitter is just a time-domain measurement of the laser's stability in its staying in phase.
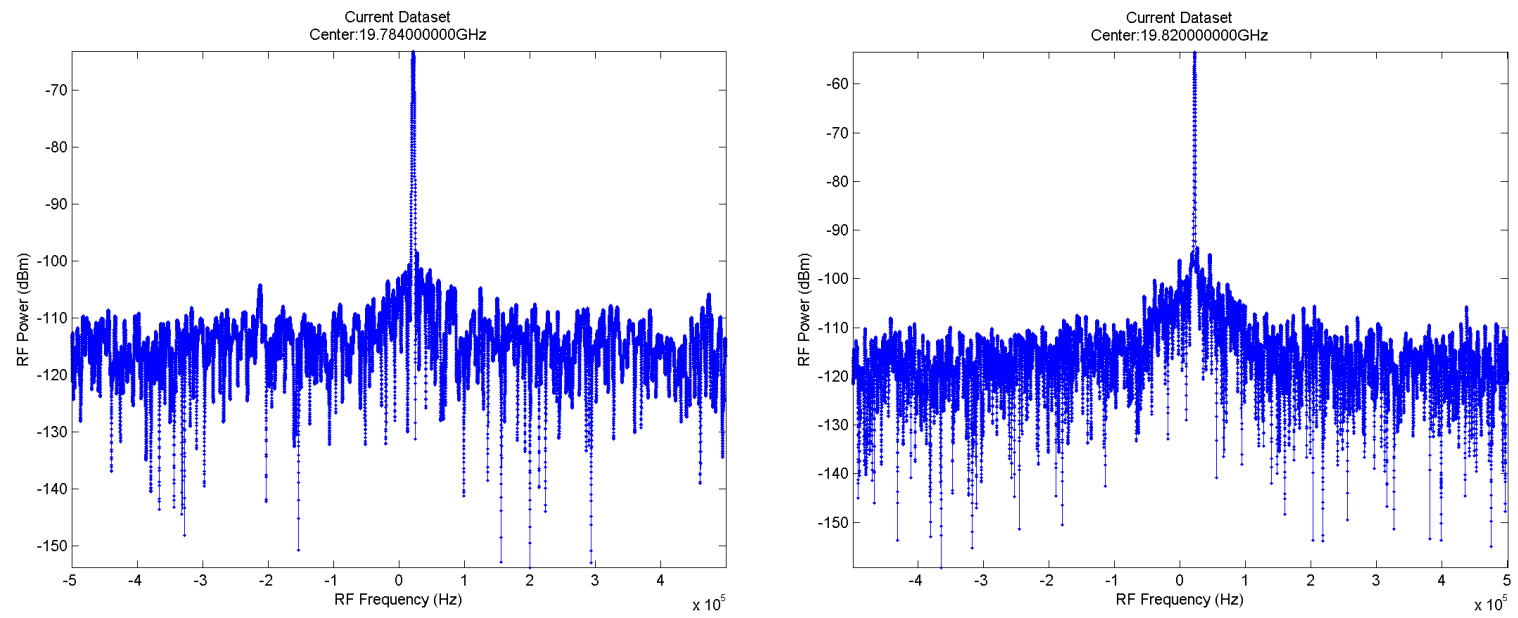

Figure 4-9: The output of the laser is measured with a high speed photodetector and an electrical spectrum analyzer. The detected spectrum is shown for the MLLD without the impedance matching network connected (left) and with (right) impedance matching network connected. The impedance matched case had a $6 \mathrm{~dB}$ higher spectral peak indicating more efficient coupling of the RF driving source to the saturable absorber segment. Resolution bandwidth $=1 \mathrm{kHz}$. 


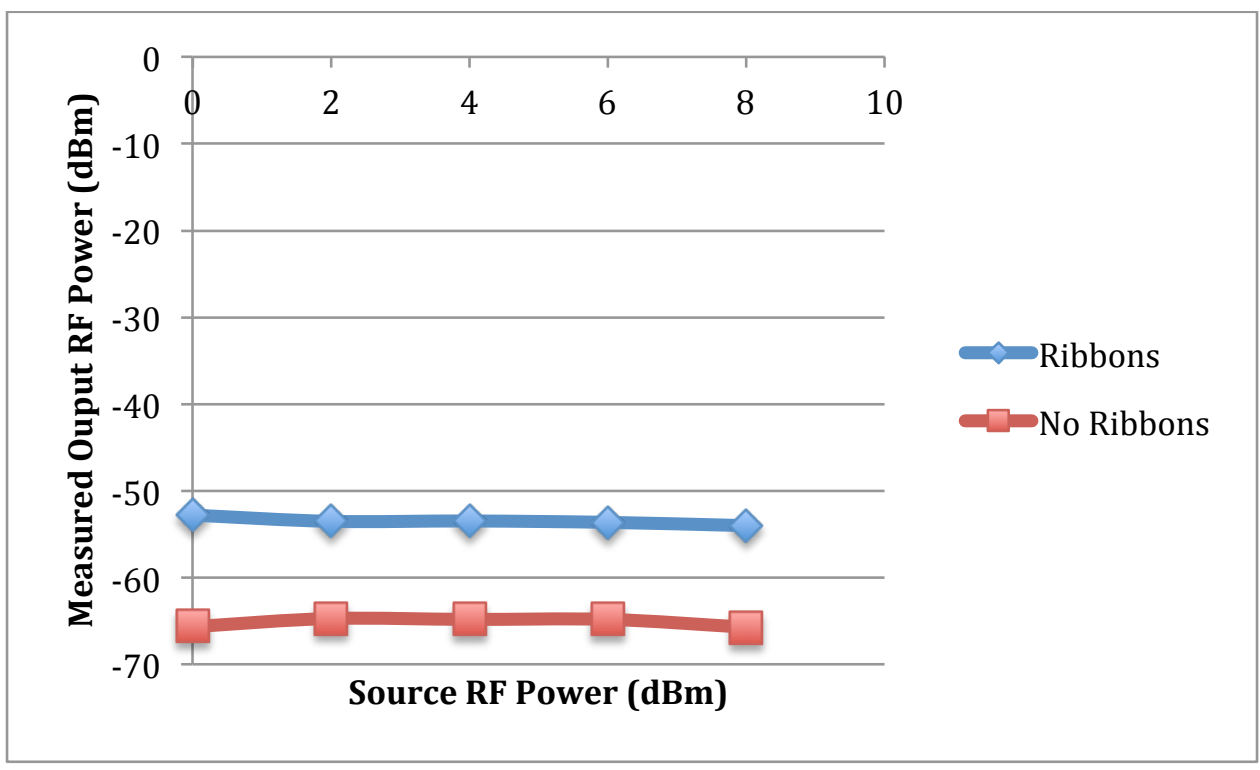

Figure 4-10: Source and measured output RF powers going into and coming out of the laser. A comparison is made between the case with ribbons attached to the center waveguide and without the ribbons attached. Once again, the impedance matched case consistently had approximately a $6 \mathrm{~dB}$ higher spectral peak under different input RF signal powers.

Now, less RF power is needed for the laser to function efficiently when being used in applications such as data transmission. This is a significant improvement in output power, in any case, and should be considered for anyone planning to use this laser as an instrument in a larger device or system in the field. In general, mode-locked lasers are of large interest in industry due to the fact that they require relatively low amounts of power to operate. With the addition of the impedance matching technique used on the laser in this thesis, this will only ensure even lower power requirements for these devices. 


\section{Chapter 5: Conclusion}

\subsection{Conclusion}

The main goal of this research project was to improve RF injection efficiency in a Hybrid Silicon mode-locked laser using impedance matching techniques. Theory and simulations show that a $14.4 \mathrm{~dB}$ improvement in RF power delivered to the diode that was initially expected. But, due to an imperfect match, and loss in the package due to non-idealities, a $6 \mathrm{~dB}$ improvement was actually made. This is still significant, as a $6 \mathrm{~dB}$ improvement in RF power means that the power delivered to the load increased by about 6 orders of magnitude. The phase noise of the laser also improved by about $10 \mathrm{~dB}$.

The laser diode was packaged in a connector module, which provided a 50 $\Omega$ transmission line between the laser and power sources. Unfortunately, because the K-Connector and the coplanar waveguide aren't connected directly, the transmission line actually ends up being quite lossy. In Between the K-Connector and the coplanar waveguide, there lies a glass bead, which contains a non-ideal dielectric constant. This slows down the propagation of microwave signals as they pass through the glass bead. The bead also extends to about 0.056 inches in length. This also contributes to a slight change in impedance in the transmission line due to resistance per unit length within the material [15].

With a passive impedance matching network inserted onto the package, a higher amount of RF power was delivered to the diode load. With more RF power being delivered to the laser diode, the diode mode-locks more productively. This 
means potentially narrower pulse widths, lower phase noise and higher peak powers. If the modulation of the saturable absorber is sycnhronized perfectly with the round trip time of the pulses in the laser cavity, it reduces the phase noise of the laser, and can also reduce the timing jitter of the pulses from the laser. As a result, the optical pulses become even shorter and more intense. [12]

This improvements are important for optical fiber communications systems, such as when laser sources are needed that for high bit data transmission or all-optical signal processing. [13] In the case of fast data transmission, semiconductor mode-locked lasers are commonly used sources for optical timedivision multiplexed (OTDM) systems. An important requirement for OTDM sources is that they have low phase noise and timing jitter. [14] As demonstrated, with impedance matching, phase noise and timing jitter are both lowered, causing for more efficient sources in OTDM applications.

Another feature of this laser, which makes it different than other typical semiconductor lasers, is its integration with electronics. Hybrid silicon lasers utilize the oxygen plasma assisted molecular wafer bonding technology, and allow III-V materials to be bonded onto Silicon-on-insulator (SOI) substrates. In the future, hundreds and possibly thousands of these lasers can be integrated onto a larger photonic integrated circuit. And with the revolutionary hybrid silicon technology, they can be integrated and interfaced with electronic circuits as well [16]. Essentially, this technology means faster and higher bandwidth optical interconnects in fiber optic communication systems, and perhaps, even future 
generation supercomputers. And, more importantly, it solves the optical interconnect bottleneck found in several of today's telecommunications applications.

\subsection{Future Work}

\subsection{1 "Active Matching"}

Another idea to consider for efficient RF injection in a mode-locked laser is this idea of an active-matching circuit. The saturable absorber of a mode-locked laser has an impedance that varies with time. When an optical pulse passes through the saturable absorber section, photogenerated carriers are created within the junction of the absorber. Those carriers create an impedance that varies with time, as they are slowly swept out of the absorber section with time.

The device structure on the saturable absorber is in essence, a p-i-n diode. The intrinsic region is an undoped zone where no space charges exist and where the quantum wells (QWs) of the absorber are located. The bias voltage applied to the saturable absorber creates a band gap shaping and distribution effect to the quantum wells known as the Quantum Confined Stark Effect (QCSE). [17] In the Quantum Confined Stark Effect, when an electric field is applied perpendicular to the surface, photo-generated holes and electrons are pulled apart, but still stay within the QWs. This applied bias causes lowering and tilting of the QWs so that the photocarriers have a shorter thermal and tunneling lifetime. A schematic of this phenomena is shown in Figure 5-1. 


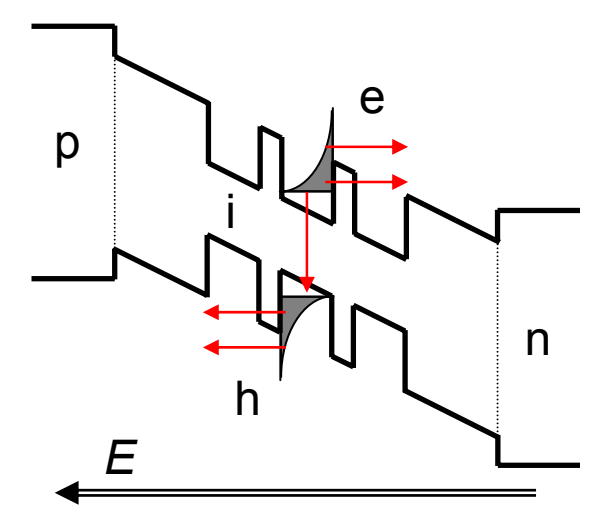

Figure 5-1: Quantum Confined Stark Effect [17]

As a result of this, the absorption recovery time experiences an exponential decay with increasing reverse bias. In other words, with larger reverse bias, the absorber recovers more quickly, and therefore, can absorb more incoming photons from incoming optical pulses. After the carriers escape the QWs, they are swept to p- and n- contacts. The transit time across the intrinsic region depends on the the region's thickness and its reverse bias. The photocurrent charges the capacitance of the structure, creating a screening potential, which partially screens the voltage applied to the absorbing layer. This actually weakens the QCSE effect, and lengthens absorber recovery time.

This is a problem because in slow saturable absorbers, such as the one as the device being used in this project, photocarriers may linger within the QWs until the next optical pulse passes through. This lengthens absorption recovery time, and photocarriers, which remain in the absorber create an impedance that varies throughout different phases of an optical pulse's round trip within the laser 
cavity. To be more specific, the photocarriers slowly sweep out of the intrinsic region into their respective $\mathrm{p}$ - and $\mathrm{n}$ - contacts and the number of photocarriers within the intrinsic region slowly decays, making a non-constant capacitance within the intrinsic region. Below is the capacitance found within the intrinsic region of the saturable absorber.

$$
C=\frac{P \alpha_{s a t}}{\hbar \omega A_{x} \varphi_{s}}
$$

$$
C=\text { capacitance across absorber's intrinsic region }
$$

$$
P=\text { average optical power }
$$

With a normal RF sine signal being used, current is injected into the absorber section in order to modulate the absorption of the absorber more accurately to actively mode-lock the hybrid silicon laser. In this case, the absorber slowly returns from forward bias to reverse bias. Carriers build up in the saturable absorber as an optical pulse passes through and light is absorbed. One way of solving this problem is to rid of these excess photocarriers as quickly as possible. If instead of using a sine signal, a pulse generator were to be used, photocarriers in the absorber QWs may be swept out in time for future incoming pulses. By rapidly applying a large reverse bias right after the optical pulse passes through, unwanted charges are quickly swept out of the absorber's intrinsic region, speeding up absorption recovery time. Now, when the next optical pulse arrives at the absorber, no leftover photocarriers remain from the previous pulse, and the absorber efficiently absorbs the succeeding incoming photons. 
In this setion, a novel wave-shaping circuit is presented to do just that. In Figure 5-2, is shown a step recovery diode (SRD) driven impulse generator, which wave-shapes the RF drive signal in order to achieve efficient injection. This circuit is included in between the input RF signal and the waveguide, which delivers power to the laser diode.

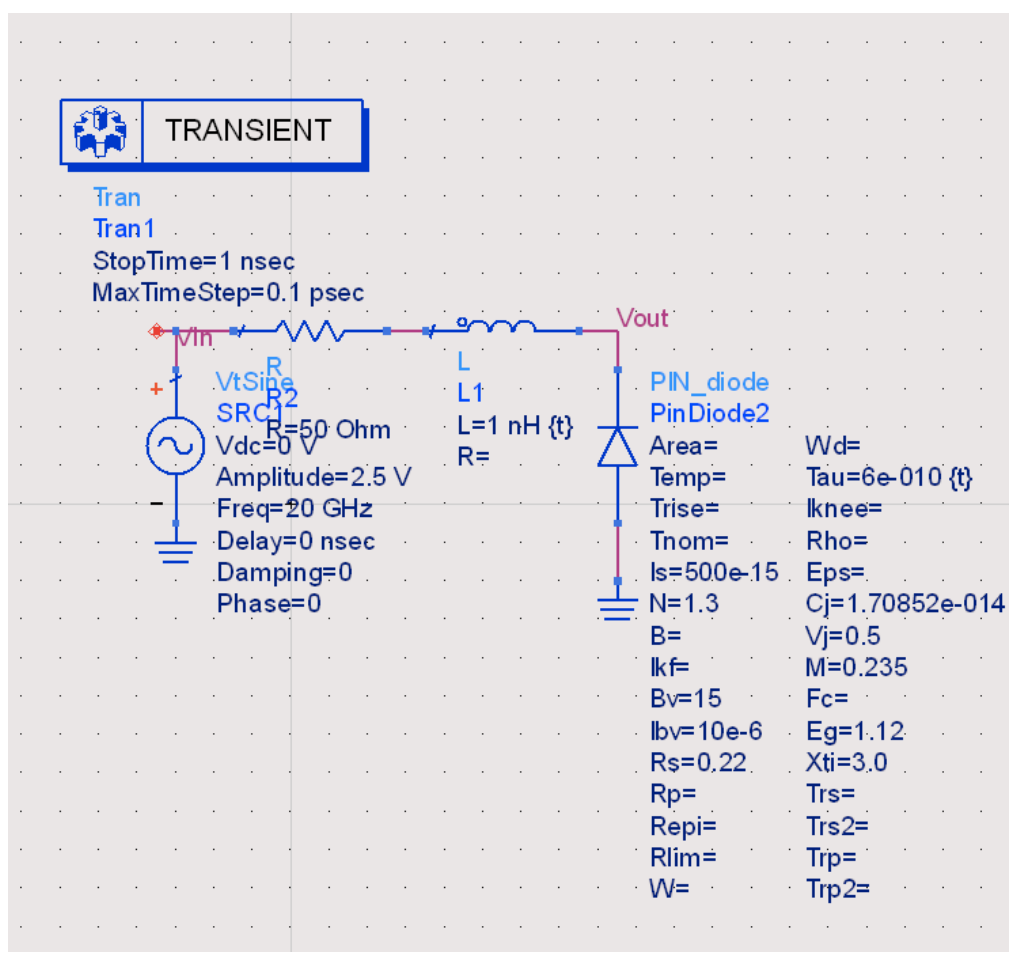

Figure 5-2: The circuit schematic for the SRD driven impulse generator 


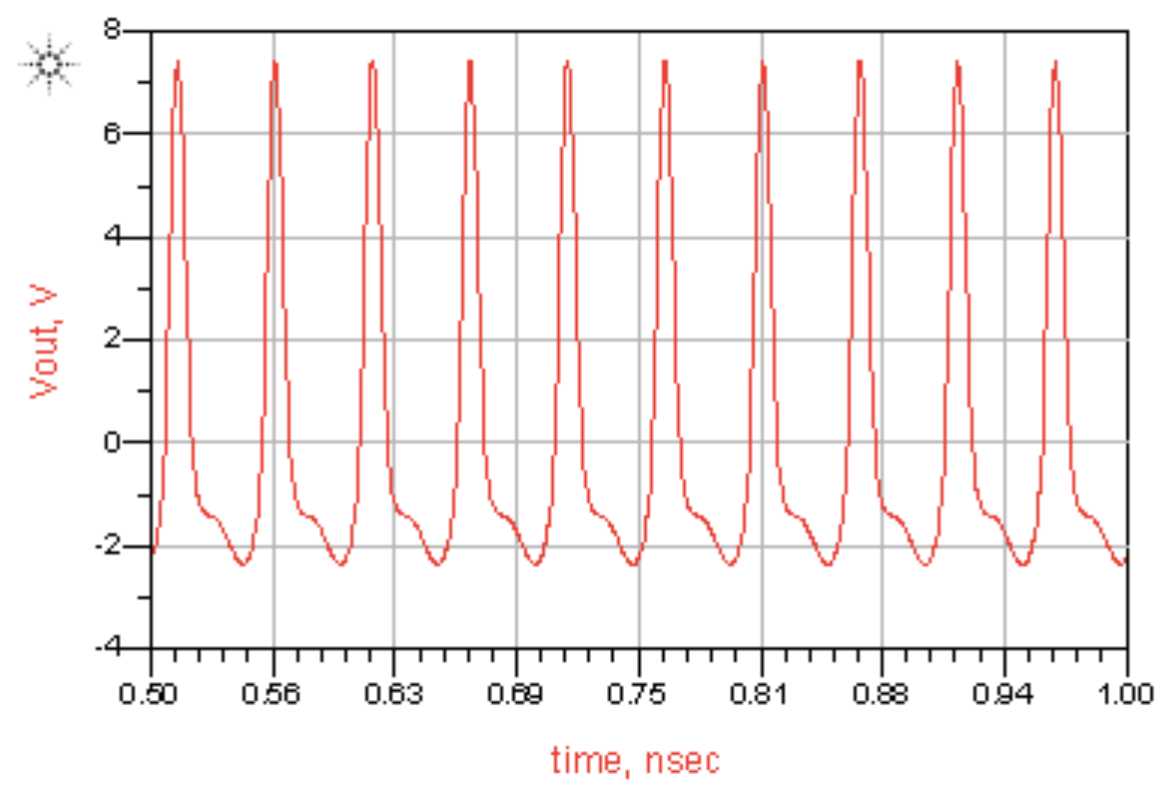

Figure 5-3: Simulated output voltage for the SRD driven impulse train generator

The key element to this circuit is the step recovery diode (SRD). The SRD is a charge-controlled switch. It is commonly used in applications such as pulse shaping, waveform generation, and frequency comb generation. Comb generators generate a number of harmonics simultaneously, which look like a comb if viewed on a spectrum analyzer. SRDs can be pulse shaping by sharpening rise time and reducing pulse widths of pulses generated by other SRDs or other components.

The way an SRD works is when charge is inserted into the SRD by forward bias, the diode appears as low impedance. Storage of charge in the SRD occurs as a result of the non-zero recombination time of minority carriers that have been injected across the junction under forward-bias. [18] When this charge 
is being removed, the diode continues as a low impedance until all the charge is removed, at which point it rapidly switches from a low impedance to a high impedance. The high impedance then stops the flow of reverse current. This means it can change impedance levels very rapidly, and can be used to generate extremely fast rise pulses, essentially mimicking the operation of a saturable absorber. This is desirable for the electric modulation signal to remove carriers from the active region of the saturable absorber during the off period of the modulation.

The circuit developed was an impulse train generator circuit, which converts the input sinusoidal RF signal into a train of impulses with very fast rise times and precisely controlled $180^{\circ}$ spacing. The way that it works is that it stores energy into an inductor before a series SRD goes from forward to reverse bias. Then the energy across the inductor causes the step-recovery diode to be driven reverse-biased very fast, getting the charge stored from the forward-biased diode capacitance to be swept out rapidly. This produces voltage spikes from rapid change in current when the diode snaps. Below is a formula for the energy stored in the inductor.

$$
\frac{1}{2} I^{2} L \cong \frac{1}{2} C E^{2}
$$

In Formula 5-2, the impulse width is shown and is determined by the resonance frequency of the $\mathrm{LC}$ circuit. By increasing the loading, the impulse height is decreased (lowering the load resistance) and the impulse width is increased. 
Formula 5-3 shows the impulse width, which is determined by the resonance frequency of the LC circuit.

$$
t \cong \pi \sqrt{L C}
$$

The SRD model used in this came circuit was a 12 picosecond transition time SRD made out of AlGaAs materials [19]. The SRD was a p-i-n double heterostructure diode with an ungraded GaAs undoped charge storage region. The zero bias capacitance of the diode was around $156 \mathrm{fF}$ and the minority carrier lifetime was around 0.53 nanoseconds. The minority carrier lifetime needs to be as short as possible in order to create shorter pulses. In fact, a minority carrier lifetime under 100 picoseconds would be ideal. The narrowest pulses produced by a SRD to date is 70 picoseconds, and so, it is possible to find a device fast enough to generate pulses on the order of $20 \mathrm{GHz}$, the operating frequency of the modelocked laser used in this project.

In conclusion, this circuit can be considered in the future as an alternative solution to the traditional passive matching network. It may be an even more clever and efficient solution to RF injection efficiency in the saturable absorber of a hybrid-silicon mode-locked laser. 


\section{REFERENCES}

[1] Bowers, J., Liang, D., Fang A., Park H., Jones R., Paniccia M., "Hybrid Silicon Lasers: The Final Frontier to Integrated Computing" in OPN Optics \& Photonics News, Massachussetts Ave, NW, 2010.

[2] Takashi, D., "Copper wires might be the bottleneck in the way of Moore's Law,"

[Online]. Available: http://venturebeat.com/2012/12/11/copper-wiresmight-be-the-bottleneck-in-the-way-of-moores-law/

[3] Coldren, L. et al., Diode Lasers and Photonic Integrated Circuits, $2^{\text {nd }}$ ed., Hoboken, New Jersey: John Wiley \& Sons, 2012.

[4] Neaman, D., Semiconductor Devices and Physics, 4th ed., Albuquerque, New Mexico: McGraw-Hill, 2011.

[5] Bhattacharya, P., Semiconductor Optoelectronic Devices, 2nd ed., New Delhi, India: Pearson Education, Inc., 1997.

[6] Heck, M., Bauters, J., Davenport, M., Doylend, J.K., Siddharth, J., Kurczveil, G., Srinivasan, S., Tang, Y., Bowers, J.E., "Hybrid Silicon Photonic Integrated Circuit Technology”, IEEE J. Sel. Topics Quantum Electron. 19, 6100117 (2013).

[7] D. Liang, G. Roelkens, R. Baets and J.E. Bowers, "Hybrid Integrated Platforms for Silicon Photonics," Materials 2010, 3, 1782-1802; doi:10.3390/ma3031782.

[8] Urzhumov, Yaroslav A.; Urzhumov, Yaroslav A (2005). "Electric and magnetic properties of sub-wavelength plasmonic crystal." Journal of Optics A: Pure and Applied Optics 7 (2): S23.

Bibcode:2005JOptA...7S..23S. doi:10.1088/1464-4258/7/2/003.

[9] Natel Engineering, "Wire Bond vs. Ribbon Bond," Natel Engineering [Online]. Available:

http://www.natelems.com/documents/dg6RBvsWB.pdf. 
[10] Microwaves101.com, "Transmission Line Loss," [Online]. Available:

http://www.microwaves 101.com/encyclopedia/Inductance_wirebond.cfm

[11] Srinivasan, S., Davenport, M., Heck, M. J. R., Hutchinson, J., Norberg, E., Fish, G., Bowers, J., "Low phase noise hybrid silicon mode-locked lasers", Springer, Frontiers of Optoelectronics, April 2014.

[12] Wikipedia, "Mode-locking," [Online]. Available: http://en.wikipedia.org/wiki/Mode_locking

[13] RP Photonics Encyclopedia, "Mode-locked Diode Lasers," [Online]. Available: http://www.rp-photonics.com/mode_locking.html

[14] Jiang, L., Ippen, E., Yokoyama, H., "Semiconductor mode-locked lasers as pulse sources for high bit rate data transmission," Journal of Optical and Fiber Communications Reports, Volume 2, Issue 1, pp 1-31, March 2005.

[15] Pozar, D., Microwave Engineering, 4th ed., Hoboken, New Jersey: John Wiley \& Sons, 2012.

[16] Chen, L., Sohdi, A., Bowers, J., Theogarajan, L., Roth, J., Fish, G., "Electronic and photonic integrated circuits for fast data center optical circuit switches," IEEE Communications Magazine Vol. 51 No. 9, September 2013.

[17] Isomaki, A. "Ultrafast Fiber Lasers Using Novel Semiconductor Saturable Absorbers and Photonic-Crystal Dispersion Compensators", Tampere University of Technology, Publication 682, October 2007.

[18] Hewlett-Packard, Appl. Note 920, pp.1-2.

[19] Hewlett-Packard, Appl. Note 918, pp.7-9. 